\title{
Resolubilidade local de campos vetoriais reais
}

\author{
Uirá Norberto Matos de Almeida
}



SERVIÇO DE PÓS-GRADUAÇÃO DO ICMC-USP

Data de Depósito:

Assinatura:

\title{
Resolubilidade local de campos vetoriais reais
}

\author{
Uirá Norberto Matos de Almeida
}

Orientador: Prof. Dr. Paulo Leandro Dattori da Silva

Dissertação apresentada ao Instituto de Ciências Matemáticas e de Computação - ICMC-USP, como parte dos requisitos para obtenção do título de Mestre em Ciências - Matemática. VERSÃO REVISADA

USP - São Carlos

Abril de 2014 
Ficha catalográfica elaborada pela Biblioteca Prof. Achille Bassi e Seção Técnica de Informática, ICMC/USP, com os dados fornecidos pelo(a) autor(a)

Matos de Almeida, Uirá Norberto
Resolubilidade local de campos vetoriais reais /
Uirá Norberto Matos de Almeida; orientador Paulo
Leandro Dattori da Silva. -- São Carlos, 2014.
121 p.
Dissertação (Mestrado - Programa de Pós-Graduação
em Matemática) -- Instituto de Ciências Matemáticas
e de Computação, Universidade de São Paulo, 2014.
1. Resolubilidade Local. 2. Campos Vetoriais. 3.
Singularidade Isolada. 4. Linearização de Sternberg.
da Silva, Paulo Leandro Dattori, orient. II.
Título.


"In re mathematica ars proponendi pluris facienda est quam solvendi."

Georg Cantor(1867) 



\section{Agradecimentos}

Agradeço primeiramente à minha família, em especial meus pais, pela força e confiança, pelo apoio incondicional e pela excelente educação que me propiciaram.

Agradeço aos meus amigos e colegas por terem tornado a minha vida ao longo desta jornada mais agradável. Em particular, agradeço aos amigos do grupo de teatro pelos momentos de descontração, e ao meu colega Erik pelas intermináveis discussões matemáticas. Um agradecimento especial ao Espeto e ao Manivela, amigos para todas as horas.

Agradeço, também, ao meu orientador Paulo pela confiança que depositou em mim.

Agradeço à FAPESP pelo apoio financeiro. 



\section{Resumo}

Nesta dissertação vamos estudar alguns importantes resultados acerca da resolubilidade local de operadores lineares de primeira ordem. Mais especificamente, seja o campo vetorial singular $L$ em $\mathbb{R}^{n}$ e dado por:

$$
L=\sum_{j=1}^{m} a_{j}(x) \frac{\partial}{\partial x_{j}} .
$$

Esta trabalho dirige-se ao estudo da resolubilidade local de $L$, isto é, dada $f \in C^{\infty}\left(\mathbb{R}^{n}\right)$ e dado $x_{0} \in \mathbb{R}^{n}$ queremos encontrar $u \in \mathcal{D}^{\prime}\left(\mathbb{R}^{n}\right)$ tal que $L u=f$ numa vizinhança de $x_{0}$. Será dada atenção especial ao caso em que os coeficientes $a_{j}(x)$ de $L$ são função lineares. Também, serão apresentados resultados sobre a resolubilidade local da equação $L u=c u+f$, sendo $c \in C^{\infty}\left(\mathbb{R}^{n}\right)$. 



\section{Abstract}

This dissertation aims to study some important results about local solvability of first order differential operators. Specifically, let $L$ be a singular vector field on $\mathbb{R}^{n}$ given by

$$
L=\sum_{j=1}^{m} a_{j}(x) \frac{\partial}{\partial x_{j}} .
$$

This work explore the local solvability of $L$, that is, given $f \in C^{\infty}\left(\mathbb{R}^{n}\right)$ and $x_{0} \in \mathbb{R}^{n}$ we want to find $u \in \mathcal{D}^{\prime}\left(\mathbb{R}^{n}\right)$ such that $L u=f$ in a neighborhood of $x_{0}$. We give special attention to the case where the coefficients $a_{j}(x)$ are linear. We also present some results about local solvability of the equation $L u=c u+f$ for $c \in C^{\infty}\left(\mathbb{R}^{n}\right)$. 



\section{Sumário}

1 Introdução 1

2 Resultados preliminares 3

3 Resolubilidade local de campos vetoriais com coeficientes reais lineares 14 3.1 Preliminares . . . . . . . . . . . . . . . . . . . . . . 14

3.2 Demonstração do Teorema . . . . . . . . . . . . . . . . . 17

3.2.1 A equivalência de (ii), (iii) e (iv) . . . . . . . . . . . . . 17

$3.2 .2 \quad$ O caso $\operatorname{dim} V(L)=n-1 \ldots \ldots \ldots . \ldots \ldots$

$3.2 .3 \quad$ O caso $m=\operatorname{codim} V(L) \geq 2 \ldots \ldots \ldots 26$

3.3 Comentários sobre uma demonstração alternativa . . . . . . . . . . . . 37

4 Resolubilidade local flat de campos vetoriais reais com singularidades isoladas $\quad 41$

5 Teorema de Linearização de Sternberg 56

6 Resolubilidade local de campos vetoriais reais com singularidade isolada 77

6.1 Resultado Principal . . . . . . . . . . . . . . . . . . 77

6.2 Demonstração do Lema $6.2 \ldots$. . . . . . . . . . . . . . . 81

6.2.1 Sobre ordenação de conjuntos . . . . . . . . . . . . . . . 81

6.2 .2 uma matriz especial . . . . . . . . . . . . . . . 83

A Geodésicas $\quad 93$

$\begin{array}{ll}\text { B Integral de Bochner } & 100\end{array}$ 
$\begin{array}{lll}\text { C Algebras de Lie } & 108\end{array}$

C.1 Noções gerais . . . . . . . . . . . . . . . . . . . . . . . 108

C.2 Demonstração do Teorema 6.6 . . . . . . . . . . . . . . . . . . . 112

C.3 Álgebra de Heisenberg e Grupo de Heisenberg . . . . . . . . . . . . . . . . 114 


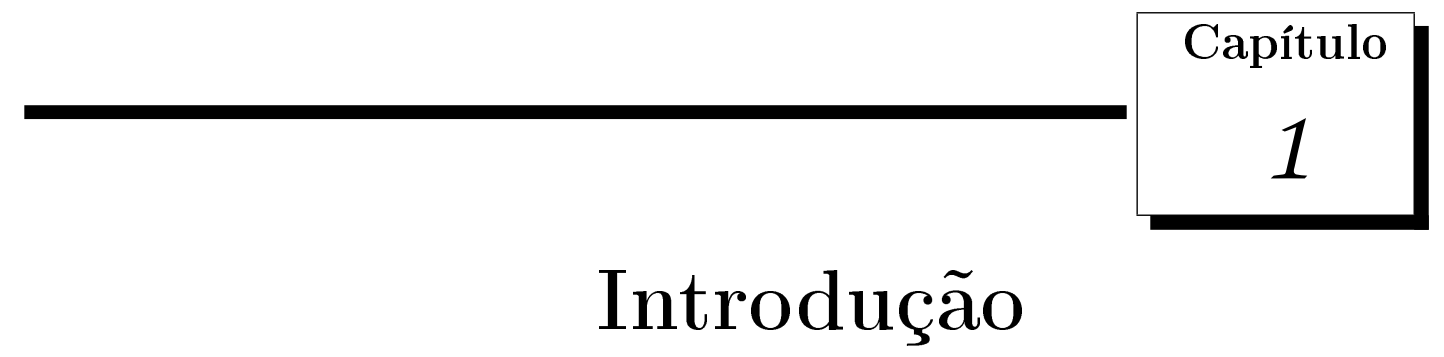

O estudo das equações diferenciais parciais tem sido, nos últimos 2 séculos, um dos grandes focos da Análise Matemática. Em particular, responder a pergunta "será que esta equação possui solução?" foi, e de fato é, uma das maiores preocupações dos matemáticos da área. Com o advento da teoria das distribuições, formalizada por Schwartz em 1950, esta questão se tornou mais abrangente, novos conceitos de solução foram introduzidos para certa classe de equações (as EDPs lineares). Em particular, faz parte desta classe, as equações obtidas como campos de vetores em variedades.

A resolubilidade local de campos vetoriais não singulares é, hoje, bem compreendida. Isso se deve, em particular, a um dos mais antigos resultados acerca dos campos de vetores, que trata da estrutura local dos campos, em torno de pontos não singulares. Mais especificamente, o resultado garante a existência de um sistema de coordenadas locais, em torno de pontos regulares, tal que, neste sistema de coordenadas, o campo de vetores é constante.

Neste trabalho, nos propusemos a estudar a resolubilidade local de campos vetoriais em torno dos pontos singulares. No capítulo 2, veremos um importante resultado sobre a sobrejetividade de operadores com coeficientes constantes. Este resultado será importante no capítulo 3, onde, seguindo o roteiro estabelecido por François Treves [Treves, 2009], faremos uma completa caracterização da resolubilidade local de campos vetoriais com coeficientes reais lineares em torno de seus pontos singulares. No capítulo 6, estudaremos o Lema 4.1.2 da tese de Maurício Fronza da Silva [Silva, 2006] e seu subsequente artigo [Silva, dos Santos Filho, 2006]. Neste capítulo, iremos nos restringir a campos 
vetoriais com singularidade isolada, mas iremos fornecer condições suficientes para que uma perturbação do campo por uma função suave tenha solução local também suave. Para melhor compreender as hipóteses e a demonstração deste resultado, os capítulos 4 e 5, apresentam demonstrações de resultados auxiliades, estabelecidos por Guillemin, Schaeffer [1977] e Sternberg [1959].

Juntos, estes resultados nos permitem ter uma visão do panorama atual no que se trata da resolubilidade local de campos de vetores com coeficientes reais. 


\section{Capítulo}

\section{Resultados preliminares}

Definição 2.1. Seja $P(D)$ um operador com coeficientes constantes. Um conjunto $\Omega \subset$ $\mathbb{R}^{n}$ é dito $P$-convexo para suporte se para todo compacto $K \subset \Omega$ existe um outro compacto $K^{\prime} \subset \Omega$ tal que

$$
\left.\begin{array}{c}
\varphi \in C_{c}^{\infty}(\Omega) \\
\operatorname{supp} P(-D) \varphi \subset K
\end{array}\right\} \Rightarrow \operatorname{supp} \varphi \subset K^{\prime} .
$$

Dizemos que $\Omega$ é $P$-convexo para suporte singular se para todo $K \subset \Omega$ existe um outro compacto $K^{\prime} \subset \Omega$ tal que

$$
\left.\begin{array}{c}
u \in \mathcal{E}^{\prime}(\Omega) \\
\text { sing } \operatorname{supp} P(-D) u \subset K
\end{array}\right\} \Rightarrow \operatorname{sing} \operatorname{supp} \varphi \subset K^{\prime} .
$$

Os seguintes resultados se encontram em Hörmander [2005] e sua referência se encontra entre parênteses.

Teorema 2.2 (Corolario 10.7.10). A equação $P(D) u=f$ possui solução $u \in \mathcal{D}^{\prime}(\Omega)$ para todo $f \in \mathcal{D}^{\prime}(\Omega)$ se, e somente se, $\Omega$ é $P$-convexo para suporte e para suporte singular.

Note que o teorema acima oferece um resultado mais forte que o teorema de Malgrange Ehrempreis, que garante apenas a resolubilidade local, porém, as condições de $P$-convexidade para suportes e para suportes singulares não são fáceis de serem trabalhadas. No que se segue, iremos seguir o roteiro estabelecido por Hormander [Hörmander, 1990], para demonstrar o seguinte resultado. 
Teorema 2.3 (Teoremas 10.6.2 e 10.7.2). Todo aberto convexo é P-convexo para suporte e para suporte singular.

Lema 2.4. Se $u \in \mathcal{E}^{\prime}\left(\mathbb{R}^{n}\right)$ então sua transformada de Fourier é dada pela função

$$
\hat{u}(\xi)=\left\langle u_{x}, e^{-2 \pi i x \cdot \xi}\right\rangle \quad, \quad \xi \in \mathbb{R}^{n} .
$$

A função $\hat{u}(\xi)$ se estende como uma função inteira em $\mathbb{C}^{n}$.

Observação 2.5. Antes de demonstrar este resultado, vamos deixar claro qual a definição de transformada de Fourier que estamos adotando:

$$
\hat{f}(\xi)=\int f(x) e^{-2 \pi i x \cdot \xi} d x
$$

Demonstração: Sejam $v \in \mathcal{E}^{\prime}(\Omega)$ e $\chi \in C_{c}^{\infty}(U), \Omega$ e $U$ abertos de $\mathbb{R}^{n}$ e $\mathbb{R}^{p}$, respectivamente, com $\chi \equiv 1$ no suporte de $v$. Sejam ainda $\phi \in C^{\infty}(\Omega \times U)$ e $u=\chi v$. Denote por $K$ o suporte de $\chi$. Então, para $y \in U$ fixado e $h \in \mathbb{R}^{p}$ suficientemente pequeno, temos da fórmula de taylor que

$$
\phi(x, y+h)=\phi(x, y)+\sum_{j=1}^{n} \frac{\partial \phi}{\partial y_{j}}(x, y) h_{j}+\psi(x, y, h)
$$

onde

$$
\psi(x, y, h)=2 \int_{0}^{1}(1-t) \sum_{|\beta|=2} \frac{1}{\beta !} \partial_{y}^{\beta} \phi(x, y+t h) h^{\beta} d t .
$$

Temos, para $x \in K$

$$
\begin{aligned}
\left|\partial_{x}^{\alpha} \psi(x, y, h)\right| & =\left|2 \int_{0}^{1}(1-t) \sum_{|\beta|=2} \frac{1}{\beta !} \partial_{x}^{\alpha} \partial_{y}^{\beta} \phi(x, y+t h) h^{\beta} d t\right| \\
& \leq 2 \int_{0}^{1}(1-t) \sum_{|\beta|=2} \frac{1}{\beta !}\left|\partial_{x}^{\alpha} \partial_{y}^{\beta} \phi(x, y+t h)\right|\left|h^{\beta}\right| d t \\
& \leq 2 \int_{0}^{1}(1-t) \sum_{|\beta|=2} \frac{1}{\beta !}\left|\partial_{x}^{\alpha} \partial_{y}^{\beta} \phi(x, y+t h)\right| h^{|\beta|} d t \leq C|h|^{2},
\end{aligned}
$$

onde $C=C(y)$, pois $K$ é compacto e podemos passar o supremo de $x \in K$ dentro da integral acima. Daí, como $u$ é distribuição de suporte compacto $K$, segue que

$$
|u(\psi(\cdot, y, h))| \leq C \sum_{|\alpha| \leq m} \sup _{x \in K}\left|\partial_{x}^{\alpha} \psi(x, y, h)\right| \leq C^{\prime}|h|^{2},
$$


onde $C^{\prime}$ só depende de $y$. Observe agora, que da linearidade de $u$ temos:

$$
u(\phi(\cdot, y+h))=u(\phi(\cdot, y))+\sum_{j=1}^{n} u\left(\frac{\partial \phi}{\partial y_{j}}(\cdot, y)\right) h_{j}+u(\psi(\cdot, y, h))
$$

Portanto, a função $y \mapsto g(y)=u(\phi(\cdot, y))$ satisfaz $g(y+h)-g(y)-T(y) h=r(y, h)$, onde $T(y)$ é linear e $|r(y, h)| \leq C^{\prime}|h|^{2}$. Isto é, provamos que $g(y)$ é diferenciável e temos:

$$
\frac{\partial}{\partial y_{j}} u_{x}\left(e^{-i \pi x \cdot y}\right)=\frac{\partial}{\partial y_{j}} g(y)=u_{x}\left(\frac{\partial}{\partial y_{j}} \phi(x, y)\right) .
$$

Repetindo o processo acima, vemos que $g(y)$ é na verdade uma função suave. Em particular, tomando $\phi(x, y)=e^{-2 \pi i x \cdot y}$ e $\varphi \in C_{c}^{\infty}\left(\mathbb{R}^{n}\right)$ qualquer,

$$
\hat{u}(\varphi)=u(\hat{\varphi})=\left(u_{x} \otimes \varphi_{\xi}\right)\left(e^{-2 \pi i x \cdot \xi}\right)=\int \varphi(\xi)\left\langle u_{x}, e^{-2 \pi i x \cdot \xi}\right\rangle d \xi=\langle g, \varphi\rangle .
$$

Finalmente, definimos a função complexa $U(\xi, \eta)=U(\zeta)=\left\langle u_{x}, e^{-2 \pi i x \cdot \zeta}\right\rangle, \zeta=\xi+\eta i$. Esta função está bem definida, pois é apenas mais um caso particular do resultado acima (estamos tomando $\phi(x, y)=e^{-2 \pi i x \cdot(\xi+\eta i)}$, onde $y=(\xi, \eta)$ ). Além disso, como já vimos, para derivar $U$ basta aplicar a distribuição $u$ à derivada de $\phi$ de modo que $U$ satisfaz a equações de Cauchy Riemman e, portanto, é holomorfa.

Definição 2.6. A extensão holomorfa da qual se trata o resultado acima é chamada de transformada de Fourier-Laplace da distribuição $u \in \mathcal{E}^{\prime}$. A transformada de Fourier-Laplace, também será denotada por $\hat{u}(\zeta)$.

Definição 2.7. Seja $K \subset \mathbb{R}^{n}$ um conjunto compacto e denote por ch $E$ o seu fecho convexo. Da versão geométrica do teorema de Hahn-Banach, temos que, se $y \notin c h K$, então podemos separar $y$ de $\operatorname{ch} K$ por um semiplano $x \cdot \xi=c$, isto é,

$$
\left\{\begin{array}{l}
x \cdot \xi<c \quad \xi \in \operatorname{ch} K \\
x \cdot y>c
\end{array}\right.
$$

Definimos a função suporte de $K$ por

$$
H_{K}(\xi)=\sup _{x \in K} x \cdot \xi=\sup _{x \in \operatorname{ch} K} x \cdot \xi
$$


Como acabamos de ver, $y \notin c h K$ implica que $y \cdot \xi>H_{K}(\xi)$.

O teorema de Paley-Wiener-Schwartz é de crucial importância para estimativas do tamanho do suporte e do suporte singular. Com fins de completeza, iremos enuncia-lo abaixo em sua versão que nos interessa.

Teorema 2.8. Sejam $K \subset \mathbb{R}^{n}$ um conjunto compacto convexo e $H_{K}$ sua função suporte.

- Uma função inteira $U(\zeta)$ é a transformada de Fourier-Laplace de uma distribuição $u \in \mathcal{E}^{\prime}\left(\mathbb{R}^{n}\right)$ com supp $u \subset K$ se, e somente se, existem constantes $C$ e $N$ tais que

$$
|U(\zeta)| \leq C(1+|\zeta|)^{N} e^{2 \pi H_{K}(\mathfrak{I m} \zeta)} \quad \forall \zeta \in \mathbb{C}^{n}
$$

- Uma função inteira $U(\zeta)$ é a transformada de Fourier-Laplace de uma função $u \in$ $C_{c}^{\infty}\left(\mathbb{R}^{n}\right)$ com supp $u \subset K$ se, e somente se, para cada $N \in \mathbb{Z}_{+}$existe constante $C_{N}$ tal que

$$
|U(\zeta)| \leq C_{N}(1+|\zeta|)^{-N} e^{2 \pi H_{K}(\mathfrak{I m} \zeta)} \quad \forall \zeta \in \mathbb{C}^{n}
$$

Iremos omitir a demonstração deste teorema, mas ele se encontra demonstrado em Hörmander [1990] ou em Hounie [1979]. Note que ele difere do resultado enunciado por Hormander por um fator de $2 \pi$ na exponencial. Isso ocorre devido à diferença na transformada de Fourier que estamos considerando.

Lema 2.9. Sejam $f \in \mathcal{E}^{\prime}$ uma distribução de suporte compacto e $P(D)$ um operador com coeficientes constantes. Então, a equação

$$
P(D) u=f
$$

possui solução $u \in \mathcal{E}^{\prime}$ se, e somente se, $\frac{\hat{f}(\zeta)}{P(\zeta)}$ é uma função inteira, sendo $P(\zeta)$ o polinômio associado ao operador $P(D)$. Além disso, esta solução satisfaz

$$
\text { ch supp } u=\operatorname{chsupp} f \text {. }
$$

Demonstração: A demonstração deste resultado será consequência do teorema de Paley-Wiener-Schwartz. Suponha que (2.0.3) tenha solução $u \in \mathcal{E}^{\prime}$. Do teorema de Paley-Wiener-Schwartz, segue que $\hat{u}$ é uma função inteira e aplicando a transformada de Fourier em ambos os lados da equação obtemos:

$$
P(\zeta) \hat{u}(\zeta)=\hat{f}(\zeta)
$$


de modo que $\frac{\hat{f}(\zeta)}{P(\zeta)}=\hat{u}(\zeta)$ é uma função inteira. Reciprocamente, se $\frac{\hat{f}(\zeta)}{P(\zeta)}$ é uma função holomorfa em $\mathbb{C}^{n}$, então, $\frac{\hat{f}(\zeta)}{P(\zeta)}=\hat{u}(\zeta)$ para alguma distribuição de suporte compacto $u$.

Para demonstrar a segunda parte do lema, vamos fazer uso da seguinte afirmação.

Afirmação: Se $h(z)$ é uma função holomorfa em $z \in \mathbb{C}$ e $p(z)=a_{m} z^{m}+\cdots+a_{1} z+a_{0}$ é um polinômio, então

$$
\left|a_{m} h(0)\right| \leq \max _{|z|=1}|h(z) p(z)|
$$

De fato, considere o polinômio $q(z)$ definido por

$$
q(z)=z^{m} \bar{p}\left(\frac{1}{z}\right)=z^{m}\left(\overline{a_{0}}+\frac{\overline{a_{1}}}{z}+\cdots+\frac{\overline{a_{m}}}{z^{m}}\right)=\overline{a_{0}} z^{m}+\overline{a_{1}} z^{m-1}+\cdots+\overline{a_{m}} .
$$

Temos que $q(0)=\overline{a_{m}}$. Note que, para $|z|=1$, vale que $z / \bar{z}=\frac{z^{2}}{|z|^{2}}=z^{2}$ e, analogamente, $\bar{z} / z=\bar{z}^{2}$; assim, para $|z|=1$,

$$
\begin{aligned}
|p(z)|^{2} & =p(z) \overline{p(z)}=\left(\sum_{j=0}^{n} a_{j} z^{j}\right)\left(\sum_{k=0}^{n} \overline{a_{k}} \bar{z}^{k}\right)=(z \bar{z})^{n}\left(\sum_{j=0}^{n} a_{j} z^{j}\right)\left(\sum_{k=0}^{n} \overline{a_{k}} \bar{z}^{k}\right) \\
& =\left(\sum_{j=0}^{n} a_{j} z^{j+n}\right)\left(\sum_{k=0}^{n} \overline{a_{k}} \bar{z}^{k+n}\right)=\left(\sum_{j=0}^{n} a_{j} z^{n-j} z^{2 j}\right)\left(\sum_{k=0}^{n} \overline{a_{k}} \bar{z}^{n-k} \bar{z}^{2 k}\right) \\
& =\left(\sum_{j=0}^{n} a_{j} z^{n-j} \frac{z^{j}}{\bar{z}^{j}}\right)\left(\sum_{k=0}^{n} \overline{a_{k}} \bar{z}^{n-k} \frac{\bar{z}^{k}}{z^{k}}\right)=\left(\sum_{j=0}^{n} a_{j} z^{n} \frac{1}{\bar{z}^{j}}\right)\left(\sum_{k=0}^{n} \overline{a_{k}} \bar{z}^{n} \frac{1}{z^{k}}\right) \\
& =z^{n} \bar{z}^{n} p\left(\frac{1}{\bar{z}}\right) \bar{p}\left(\frac{1}{z}\right)=|\bar{p}(1 / z)| .
\end{aligned}
$$

Portanto, segue do princípio do módulo máximo que

$$
\left|a_{m} h(0)\right|=|q(0) h(0)| \leq \max _{|z|=1}|q(z) h(z)|=\max _{|z|=1}\left|z^{m} \bar{p}\left(\frac{1}{z}\right) h(z)\right|=\max _{|z|=1}|p(z) h(z)|
$$

finalizando a demonstração da afirmação.

Voltando, agora, para demonstração do lema, temos que é possível encontrar coordenadas tal que o coeficiente $a$ de $\zeta_{1}^{m}$ em $P(\zeta)$ é não nulo, sendo $m$ a ordem do operador $P(D)$. Então, considerando $P\left(\zeta_{1}+z, \zeta_{2}, \ldots, \zeta_{n}\right)$ um polinômio em $z$, temos que 
seu coeficiente líder ${ }^{1}$ é $a$. Suponha que $u$ seja a solução de (2.0.3). Então, $P(\zeta) \hat{u}(\zeta)=\hat{f}(\zeta)$ e da afirmação, temos que

$$
\begin{aligned}
|a \hat{u}(\zeta)| & \leq \sup _{|z|=1}\left|P\left(\zeta_{1}+z, \zeta_{2}, \ldots, \zeta_{n}\right) \hat{u}\left(\zeta_{1}+z, \zeta_{2}, \ldots, \zeta_{n}\right)\right| \\
& =\sup _{|z|=1}\left|\hat{f}\left(\zeta_{1}+z, \zeta_{2}, \ldots, \zeta_{n}\right)\right|
\end{aligned}
$$

portanto, se $\hat{f}$ satisfaz a estimativa (2.0.1) para constantes $C$ e $N$, segue que $\hat{u}$ também as satisfaz, apenas para uma outra constante $C^{\prime}$ no lugar de $C$. Do teorema de Paley-Wiener-Schwartz, isso implica que $u \in \mathcal{E}^{\prime}$, e como as estimativas são as mesmas, exceto pela constante, segue que

$$
\text { ch supp } u \subset \text { ch supp } f \text {. }
$$

A inclusão contrária é trivial.

Corolário 2.10. Todo aberto convexo $U$ é $P$-convexo para suporte.

Demonstração: Seja $K$ um compacto em $U$ e tome $K^{\prime}=c h K$. Segue do lema que

$$
\operatorname{ch} \operatorname{supp} P(D) \varphi=\operatorname{ch} \operatorname{supp} \varphi
$$

$\log 0$

$$
\operatorname{supp} P(D) \varphi \subset K \Rightarrow \operatorname{supp} \varphi \subset K^{\prime}
$$

Para provar que a convexidade implica $P$-convexidade para suporte singular iremos precisar do seguinte análogo singular do teorema de Paley-Wiener-Schwartz.

Teorema 2.11. Seja $u \in \mathcal{E}^{\prime}\left(\mathbb{R}^{n}\right)$ e seja $K \subset \mathbb{R}^{n}$ um compacto convexo. Então, para que sing supp $u \subset K$, é necessário e suficiente que exista uma constante $N$ e uma sequência de constantes $C_{m}, m=1,2, \ldots$ tais que

$$
|\hat{u}(\zeta)| \leq C_{m}(1+|\zeta|)^{N} e^{2 \pi H(\mathfrak{I m} \zeta)} \text { se }|\mathfrak{I m}(\zeta)| \leq \frac{m}{2 \pi} \log (|\zeta|+1) \quad \zeta \in \mathbb{C} .
$$

\footnotetext{
${ }^{1}$ Coeficiente do termo de maior grau. Neste caso, o termo $z^{m}$
} 
Demonstração: Vamos provar que a condição do teorema é necessária:

Fixe $m \in \mathbb{Z}_{+}$e seja $K_{m}=\left\{x \in \mathbb{R}^{n} ;|x-K| \leq 1 / m\right\}$. Observe que, se $H_{K_{m}}$ é a função de suporte de $K_{m}$, então

$$
\begin{aligned}
H_{K_{m}}(\zeta) & =\sup _{x \in K_{m}}\{x \cdot \zeta\} \leq \sup _{x \in K,|y| \leq 1 / m}\{(x+y) \cdot \zeta\} \\
& \leq \sup _{x \in K}\{x \cdot \zeta\}+\sup _{|y|<1 / m}\{y \cdot \zeta\} \leq H_{K}(\zeta)+\frac{|\zeta|}{m}
\end{aligned}
$$

Consideremos uma decomposição de $u$ da seguinte forma:

$$
u=u_{1}+u_{2} \quad \text { tais que } \operatorname{supp} u_{1} \subset K_{m} ; u_{2} \in C_{c}^{\infty} .
$$

Esta decomposição é possível pois o suporte singular de $u$ está contida em $K_{m}$. Daí, segue do teorema de Paley-Wiener-Schwartz que se $u_{1}$ satisfaz a estimativa (2.0.1) para constantes $N-1$ e $C_{m}$ então

$$
\left|\hat{u}_{1}(\zeta)\right| \leq C_{m}(1+|\zeta|)^{N-1} e^{2 \pi H_{K_{m}}(\mathfrak{I m} \zeta)} \leq C_{m}(1+|\zeta|)^{N-1} e^{\frac{2 \pi}{m}|\mathfrak{I m} \zeta|} e^{2 \pi H_{K}(\mathfrak{I m} \zeta)}
$$

Supondo que $|\mathfrak{I m}(\zeta)| \leq m \log (1+|\zeta|)$, temos:

$$
\left|\hat{u}_{1}(\zeta)\right| \leq C_{m}(1+|\zeta|)^{N-1} e^{\frac{2 \pi}{m}\left(\frac{m}{2 \pi} \log (1+|\zeta|)\right)} e^{2 \pi H_{K}(\mathfrak{I m} \zeta)}=C_{m}(1+|\zeta|)^{N} e^{2 \pi H_{K}(\mathfrak{I m} \zeta)} .
$$

Por outro lado, segue novamente do teorema de Paley-Wiener-Schwartz, que $u_{2}$ satisfaz a estimativa

$$
\left|\hat{u}_{2}(\zeta)\right| \leq C_{N}(1+|\zeta|)^{-N} e^{2 \pi H_{A}(\mathfrak{I m} \zeta)} \quad \forall \zeta \in \mathbb{C}^{n}
$$

onde $A$ é um compacto convexo tal que supp $u_{2} \subset A$. Note que

$$
H_{K}(\xi)=\sup _{x \in K}\{x \cdot \xi\} \leq \sup _{x \in K}|x||\xi| \leq\|K\||\xi|
$$

onde $\|K\|$ denota o diametro do conjunto $K$. Observe ainda que

$$
-H_{K}(\xi)=-\sup _{x \in K}\{x \cdot \xi\}=\inf _{x \in K}\{-x \cdot \xi\} \leq|\zeta|\left|\inf _{x \in K}\right| x \mid .
$$


Daí, supondo que $|\mathfrak{I m}(\zeta)| \leq m \log (1+|\zeta|)$ e denotando por $r=\inf _{x \in K}|x|$, temos que

$$
\begin{aligned}
\left|\hat{u}_{2}(\zeta) e^{-2 \pi H_{K}(\mathfrak{I m} \zeta)}\right| & \leq C_{N}(1+|\zeta|)^{-N} e^{2 \pi\left(H_{A}-H\right)(\mathfrak{I m} \zeta)} \leq C_{N}(1+|\zeta|)^{-N} e^{2 \pi(\|A\|+r)|\mathfrak{I m} \zeta|} \\
& \leq C_{N}(1+|\zeta|)^{-N}(1+|\zeta|)^{2 \pi(\|A\|+r) \frac{m}{2 \pi}} \leq C_{N}(1+|\zeta|)^{(A+r) m-N}
\end{aligned}
$$

Como a estimativa acima é válida para todo $N$, segue que $\left|\hat{u}_{2}(\zeta) e^{-2 \pi H(\mathfrak{I m} \zeta)}\right| \rightarrow 0$ quando $|\zeta| \rightarrow \infty$. Logo vale a estimativa

$$
\left|\hat{u}_{2}(\zeta)\right| \leq \tilde{C}_{m}(1+|\zeta|)^{N} e^{2 \pi H_{K}(\mathfrak{I m} \zeta)} \quad ; \quad|\mathfrak{I m}(\zeta)| \leq \frac{m}{2 \pi} \log (1+|\zeta|),
$$

para alguma constante $\tilde{C}_{m}$. Daí, para $|\mathfrak{I m}(\zeta)| \leq \frac{m}{2 \pi} \log (1+|\zeta|)$, temos

$$
|\hat{u}(\zeta)| \leq\left|\hat{u}_{1}(\zeta)\right|+\left|\hat{u}_{2}(\zeta)\right| \leq\left(C_{m}+\tilde{C}_{m}\right)(1+|\zeta|)^{N} e^{2 \pi H_{K}(\mathfrak{I m} \zeta)}
$$

obtendo a estimativa desejada.

Reciprocamente, para cada $\eta \in \mathbb{R}^{n}$, defina

$$
\mathbb{R}^{n} \ni \xi \mapsto \zeta(\xi)=\xi+i \eta \log \left(1+|\xi|^{2}\right)
$$

Seja então $\Gamma_{\eta}=\zeta\left(\mathbb{R}^{n}\right)$.

Considere a seguinte região:

$$
\Omega_{\eta}=\left\{x+t i \eta \log \left(1+|x|^{2}\right) \in \mathbb{C}^{n} ; x \in \mathbb{R}^{n} ; t \in(0,1)\right\}
$$

Notemos primeiramente que $\Omega_{\eta}$ está contido em um subespaço vetorial real $V$ de $\mathbb{C}^{n}$. De fato, temos que

$$
x+t i \eta \log \left(1+|x|^{2}\right) \subset \mathbb{R}^{n} \oplus i \eta \mathbb{R} \equiv V .
$$

É fácil ver que $\Omega_{\eta}$ é aberto em $V$. Além disso, a fronteira de $\Omega_{\eta}$ é o conjunto $\Gamma_{0} \cup \Gamma_{\eta}$.

Seja agora $\varphi$ função de suporte compacto na bola unitária e tal que $\int \varphi d x=1$. Defina então $\varphi_{\varepsilon}=\varepsilon^{-n} \varphi(x / \varepsilon)$. Defina ainda $g(w)=e^{2 \pi i x \cdot w} \hat{u}(w) \hat{\varphi}_{\varepsilon}(w)$. 
Observe que

$$
1+|\xi|^{2}=(1+i|\xi|)(1-i|\xi|)<(1+|\zeta(\xi)|)^{2}
$$

$\log \mathrm{o}$

$$
|\mathfrak{I m}(\zeta(\xi))| \leq 2|\eta| \log (1+|\zeta|)
$$

em particular, para cada $\eta$ fixado, temos que existe constante $C(\eta)$ tal que $\zeta(\xi)$ satisfaz a estimativa (2.0.4), isto é,

$$
\begin{aligned}
|\hat{u}(\zeta(\xi))| & \leq C_{\eta}(1+|\zeta(\xi)|)^{N} e^{2 \pi H(\mathfrak{I m} \zeta(\xi))} \\
& \leq C_{\eta}(1+|\zeta|)^{N} e^{2 \pi\|K\||| \mathfrak{m} \zeta \mid} \leq C_{\eta}(1+|\zeta|)^{N} e^{4 \pi\|K\||\eta| \log (1+|\zeta|)} \\
& =C_{\eta}(1+|\zeta|)^{N} e^{\log (1+|\zeta|)^{4 \pi\|K\||| \eta \mid}}=C_{\eta}(1+|\zeta|)^{N}(1+|\zeta|)^{4 \pi\|K\||\eta|} \\
& =C_{\eta}(1+|\zeta|)^{N+4 \pi\|K\||| \eta \mid}
\end{aligned}
$$

Assim, segue de (2.0.5) e do teorema de Paley-Wiener-Schwartz que $g$ satisfaz, para cada $N_{2} \in \mathbb{Z}_{+}$, uma estimativa do tipo

$$
\begin{aligned}
|g(\zeta)| & \leq e^{-2 \pi x \cdot \mathfrak{I m}(\zeta)} C_{\eta}(1+|\zeta|)^{N_{1}+4 \pi\|K\||\eta|} C_{N_{2}}(1+|\zeta|)^{-N_{2}+4 \pi\|K\||\eta|} \\
& =e^{\log \left(1+|\xi|^{2}\right)^{-2 \pi x \cdot \eta}} C_{\eta}(1+|\zeta|)^{N_{1}+4 \pi\|K\||\eta|} C_{N_{2}}(1+|\zeta|)^{-N_{2}+4 \pi\|K\||\eta|} \\
& =C_{\eta} C_{N_{2}}\left(1+|\xi|^{2}\right)^{-2 \pi x \cdot \eta}(1+|\zeta|)^{-N_{2}+N_{1}+8 \pi\|K\| \mid \eta \eta}
\end{aligned}
$$

para todo $\zeta \in \Omega_{\eta}$. Observe ainda que $\Gamma_{0}$ e $\Gamma_{\eta}$ são $n$-ciclos homotópicos em $V$, logo são cíclos homólogos. Segue do teorema de Stokes $^{2}$ que

$$
\int_{\mathbb{R}^{n}} g(x) d x=\int_{\Gamma_{0}} g(\zeta) d \zeta=\int_{\Gamma_{\eta}} g(\zeta) d \zeta
$$

onde a existência da integral da direita é garantida pela estimativa (2.0.7) e a existência da integral da esquerda é garantida pela seguinte conta

$$
u * \varphi_{\varepsilon}(x)=\mathcal{F}^{-1}\left(\widehat{u * \varphi_{\varepsilon}}\right)(x)=\mathcal{F}^{-1}\left(\hat{u} \hat{\varphi}_{\varepsilon}\right)(x)=\int_{\mathbb{R}^{n}} g(y) d y .
$$

\footnotetext{
${ }^{2} \mathrm{O}$ teorema de Stokes garante que a integral $\int_{z} w$ depende apenas das classes de cohomologia da forma fechada $w$ e da classe de homologia do ciclo $z$. Ver Eschrig [2011], capítulo 5 .
} 
Observe agora que, para $\zeta=\zeta(\xi)$, com $\xi \in \mathbb{R}^{n}$, temos:

$$
\begin{aligned}
\left|e^{2 \pi i x \cdot \zeta} \hat{u}(\zeta)\right| & \leq e^{-2 \pi x \cdot \mathfrak{I m} \zeta} C_{\eta}(1+|\zeta(\xi)|)^{N} e^{2 \pi H(\mathfrak{I m} \zeta(\xi))} \\
& =e^{-2 \pi x \cdot \eta \log \left(1+|\xi|^{2}\right)} C_{\eta}(1+|\zeta(\xi)|)^{N} e^{2 \pi H(\mathfrak{I m} \zeta(\xi))} \\
& =C_{\eta}\left(1+|\xi|^{2}\right)^{-2 \pi x \cdot \eta}(1+|\zeta(\xi)|)^{N} e^{2 \pi H(\mathfrak{I m} \zeta)} .
\end{aligned}
$$

Note que $H(\mathfrak{I m} \zeta)=\sup _{y \in K}\left\{y \cdot \eta \log \left(1+|\xi|^{2}\right)\right\}=H(\eta) \log \left(1+|\xi|^{2}\right)$. Daí,

$$
\begin{aligned}
\left|e^{2 \pi i x \cdot \zeta} \hat{u}(\zeta)\right| & \leq C_{\eta}\left(1+|\xi|^{2}\right)^{2 \pi H(\eta)-2 \pi x \cdot \eta}(1+|\zeta(\xi)|)^{N} \\
& =C_{\eta}\left(1+|\xi|^{2}\right)^{2 \pi(H(\eta)-x \cdot \eta)}(1+|\zeta(\xi)|)^{N}
\end{aligned}
$$

Lembrando que, se $y \notin c h K$, então podemos separar $y$ de $\operatorname{ch} K$ por um semiplano $x \cdot \xi=c$, então, dado $x_{0} \notin K$, podemos escolher $\eta$ de modo que, em uma vizinhança $B_{0}$ de $x_{0}, B_{0} \cap \operatorname{ch} K=\emptyset$, temos que

$$
0>-c=H_{K}(\eta)-x \cdot \eta \quad \forall x \in B_{0},
$$

sendo $c>0$. Como $H(t \xi)=t H(\xi)$ segue que, para $t>0$ temos:

$$
0>-t c=H_{K}(t \eta)-x \cdot t \eta \quad \forall x \in B_{0}
$$

ou seja, para cada $M \in \mathbb{Z}_{+}$é possivel escolher $\tilde{\eta}=t \eta$ com $t$ suficientemente grande tal que

$$
\left|e^{2 \pi i x \cdot \zeta} \hat{u}(\zeta)\right| \leq C_{\eta}\left(1+|\xi|^{2}\right)^{-M}(1+|\zeta(\xi)|)^{N} \quad \forall x \in B_{0}
$$

Em particular, isto implica que $e^{2 \pi i x \cdot \zeta} \hat{u}(\zeta)$ é integrável e, para $x \in B_{0}$, podemos tomar o limite

$$
u(x)=\lim _{\varepsilon \rightarrow 0} u * \varphi_{\varepsilon}(x)=\lim _{\varepsilon \rightarrow 0} \int_{\Gamma_{\tilde{\eta}}} e^{2 \pi i x \cdot \zeta} \hat{u}(\zeta) \hat{\varphi}_{\varepsilon}(\zeta) d \zeta=\int_{\Gamma_{\tilde{\eta}}} e^{2 \pi i x \cdot \zeta} \hat{u}(\zeta) d \zeta .
$$

Mais ainda, para cada $j$ fixado, é possível tomar $M$ tal que o limite acima ainda pode ser tomado, mesmo após $j$ derivações. Assim, $u$ é $C^{j}\left(\mathbb{R}^{n}\right)$. Da arbitrariedade de $j$, segue que $u$ é suave em $B_{0}$. Da arbitrariedade de $x$, segue que $u$ é suave em $\mathbb{C} \backslash c h K$, de modo que sing supp $u \subset K$, conforme queríamos demonstrar. 
Podemos, então, considerar um resultado análogo ao Lema 2.9 para suporte singular.

Teorema 2.12. Se $u \in \mathcal{E}^{\prime}\left(\mathbb{R}^{n}\right)$ ent $\tilde{a} O$

$$
\text { ch sing supp } u=\text { ch sing supp } P(D) u \text {. }
$$

\section{Demonstração:}

Seja $f=P(D) u$ e seja $K=$ chsingsupp $f$. Fazendo como na demonstração do Lema 2.9, fazemos uma mudança ortogonal de coordenadas de modo que o termo líder do polinômio $P(\zeta)$ seja $a \zeta_{1}^{m}$ obtemos que

$$
\begin{aligned}
|a \hat{u}(\zeta)| & \leq \sup _{|z|=1}\left|P\left(\zeta_{1}+z, \zeta_{2}, \ldots, \zeta_{n}\right) \hat{u}\left(\zeta_{1}+z, \zeta_{2}, \ldots, \zeta_{n}\right)\right| \\
& =\sup _{|z|=1}\left|\hat{f}\left(\zeta_{1}+z, \zeta_{2}, \ldots, \zeta_{n}\right)\right|
\end{aligned}
$$

sendo a o coeficiente líder do polinômio $P(\zeta)$.

Daí, se $\hat{f}$ satisfaz a estimativa (2.0.4) então $u$ também satisfaz esta estimativa para uma outra constante e, portanto,

$$
\text { ch sing supp } u \subset K \text {. }
$$

A inclusão contrária é trivial, pois o operador $P(D)$ "encolhe"o suporte singular.

O seguinte corolário tem demonstração análoga à demonstração do Corolário 2.10.

Corolário 2.13. Abertos convexos são $P$-convexos para suporte singular.

Está, então, demonstrado o Teorema 2.3. 


\section{Capítulo}

Resolubilidade local de campos vetoriais com coeficientes reais lineares

\subsection{Preliminares}

Consideremos em $\mathbb{R}^{n}$ um campo de vetores suave, cujos coeficientes sejam funcionais lineares reais, isto é, um campo de vetores da forma:

$$
L=\sum_{j, k=1}^{n} a_{j k} x_{j} \frac{\partial}{\partial x_{k}} .
$$

Definição 3.1. Um campo de vetores suave é dito ser localmente resolúvel em um aberto $\Omega \subset \mathbb{R}$, se cada ponto $x_{0} \in \Omega$ possui uma vizinhança aberta $U \subset \Omega$ tal que, para todo $f \in C_{0}^{\infty}(U)$, existe $u \in \mathcal{D}^{\prime}(\Omega)$ que satisfaz $L u=f$ em $U$.

Iremos agora estabelecer condições que são equivalentes à resolubilidade (mais precisamente, à não-resolubilidade) local na origem de campos do tipo (3.1.1). Veremos ainda que a resolubilidade local na origem é equivalente à resolubilidade local em cada ponto crítico para campos deste tipo.

Vamos denotar por $m(L)$ a matriz real $\left(a_{j k}\right)_{j, k=1, \ldots, n}$. Lembrando que o divergente de um campo vetorial $X=\sum b_{j}(x) \partial_{x_{j}}$ é dado por

$$
\operatorname{div}(X)=\sum \frac{\partial b_{j}}{\partial x_{j}}(x)
$$


temos que

$$
\operatorname{div}(L)=\sum_{k=1}^{n} \frac{\partial}{\partial x_{k}}\left(\sum_{j=1}^{n} a_{j k} x_{j}\right)=\sum_{k=1}^{n} a_{k k}=\operatorname{Tr}(m(L))
$$

Os pontos críticos de $L$, denotado por $V(L)$, são os pontos $\left(x_{1}, \ldots, x_{n}\right) \in \mathbb{R}^{n}$ tais que $m(L)^{\top} x=\overrightarrow{0}$. $V(L)$ é um subespaço vetorial de $\mathbb{R}^{n}$. Podemos escolher um sistema de coordenadas em que $V(L)=\left\{x \in \mathbb{R}^{n} ; x_{1}=\cdots=x_{m}=0\right\}$, sendo $m$ a codimensão de $V(L)$. Neste sistema de coordenadas escrevemos o campo de vetores (3.1.1) da seguinte forma:

$$
L=\sum_{j=1}^{m} \sum_{k=1}^{n} a_{j k} x_{j} \frac{\partial}{\partial x_{k}} .
$$

Observe que o campo de vetores (3.1.2) é invariante por translações nas coordenadas $x_{m+1}, \ldots, x_{n}$, e que podemos escrever:

$$
L=\sum_{j=1}^{m} x_{j} L_{j} ; \quad L_{j}=\sum_{k=1}^{n} a_{j k} \frac{\partial}{\partial x_{k}}
$$

Como $m$ é exatamente a codimensão de $V(L)$, então, as primeiras $m$ linhas da matriz $m(L)$ são $L . I$ e, portanto, os campos de vetores $L_{1}, \ldots, L_{m}$ também são $L . I$.

Consideremos a projeção

$$
\begin{aligned}
\pi: \mathbb{R}^{n} & \longrightarrow \mathbb{R}^{n} / V(L) \\
x=\left(x_{1}, \ldots, x_{n}\right) & \mapsto\left(y_{1}, \ldots, y_{m}\right) ; y_{j}(x)=x_{j},
\end{aligned}
$$

onde $\mathbb{R}^{n} / V(L)$ é identificado ${ }^{1}$ de maneira natural com $\mathbb{R}^{m}$. Podemos agora, através do pushforward $^{2}$, associar o campo vetorial $L$ (na forma (3.1.2)) com o campo vetorial em $\mathbb{R}^{m}$

$$
L^{0}=\pi_{*} L=\sum_{j=1}^{m} y_{j} \sum_{k=1}^{m} L\left(y_{k}\right) \frac{\partial}{\partial y_{k}}=\sum_{j, k=1}^{m} a_{j k} y_{j} \frac{\partial}{\partial y_{k}}
$$

\footnotetext{
${ }^{1}$ Estamos aqui considerando um sistema de coordenadas tal que $V(L)=\left\{\vec{x} \in \mathbb{R}^{n} ; x_{1}=\cdots=x_{m}=0\right\}$.

${ }^{2}$ Se $x=\left(x_{1}, \ldots, x_{n}\right) \mapsto \varphi(x)=\left(y_{1}, \ldots, y_{m}\right)$ é suave, então o pushforward do campo de vetores $X$ por $\varphi$ no ponto $q=\varphi(p)$ é definido por $\varphi_{*} X(q)=(d \varphi)_{p} X(p)$. Note que quando $n \neq m$, o pushforward pode não esta bem definido, mas em nosso caso, $(d \pi)_{p}$ independe de $p$ pois $\pi$ é linear e, além disso, $\pi\left(p_{1}\right)=\pi\left(p_{2}\right)$ implica que $p_{1}-p_{2} \in V(L)$ e assim, $L\left(p_{1}\right)=L\left(p_{2}\right)$ e, portanto, o pushforward $\pi_{*} L$ de $L$ pela projeção está bem definido.
} 
Observe que o polinômio característico de $m(L)$ é dado por

$$
P_{m(L)}(\lambda)=\operatorname{det}\left(m(L)-\lambda I_{n}\right)
$$

onde $I_{n}$ denota a matriz identidade de dimensões $n \times n$, mas

$$
m(L)-\lambda I_{n}=\left(\begin{array}{ccc}
m\left(L^{0}\right)-\lambda I_{m} & \vdots & \star \\
\ldots \ldots \ldots \ldots \ldots \ldots \ldots & \vdots & \ldots \ldots \ldots \ldots \ldots \\
0 & \vdots & -\lambda I_{m-n}
\end{array}\right)
$$

e, portanto, $\operatorname{det}\left(m(L)-\lambda I_{n \times n}\right)=(-\lambda)^{n-m} \operatorname{det}\left(m\left(L^{0}\right)-\lambda I_{m \times m}\right)$. Logo, o espectro de $m(L)$ e $m\left(L^{0}\right)$ coincidem, exceto pelo auto-valor zero, isto é $\sigma(m(L)) \backslash\{0\}=\sigma\left(m\left(L^{0}\right)\right) \backslash\{0\}$. Em particular, temos que $\operatorname{div}(L)=\operatorname{div}\left(L^{0}\right)$.

Observe que $L$ define uma folheação ${ }^{3} \mathrm{em} \mathbb{R}^{n}$, onde as folhas são justamente os pontos de $V(L)$ ou curvas "de verdade" em $\mathbb{R}^{n} \backslash V(L)$. Mais precisamente, são variedades unidimensionais imersas, conexas, analíticas e sem auto-interseção, cujas linhas tangentes são geradas por $L$ em cada ponto (e mais, as folhas em $\mathbb{R}^{n} \backslash V(L)$ são maximais com essas propriedades). Essas folhas serão chamadas de $L$-órbitas.

Podemos agora enunciar o resultado principal desta seção, que irá caracterizar a resolubilidade local dos campos (3.1.1). Este resultado foi enunciado e demonstrado por Treves [2009]. Aqui, apresentaremos em detalhes a demonstração dada por Treves.

Teorema 3.2. As seguintes propriedades do campo de vetores (3.1.1) são equivalentes:

(i) L não é localmente resolúvel em nenhum ponto de $V(L)$;

(ii) O fecho de qualquer L-órbita em $\mathbb{R}^{n} \backslash V(L)$ é compacto em $\mathbb{R}^{n} \backslash V(L)$;

(iii) O espectro de $m\left(L^{0}\right)$ é puramente imaginário e sua parte nilpotente é nula;

(iv) $V(L)$ tem codimensão $m=2 p$ e existe um sistema de coordenadas $x_{1}, \ldots, x_{n}$ tal que qualquer L-órbita por um ponto $x^{0} \in \mathbb{R}^{n} \backslash V(L)$ é um geodésica do toro $(\tilde{T})^{p}\left(x^{0}\right)$ dado por:

$$
(\tilde{T})^{p}\left(x^{0}\right):=\left\{\begin{array}{l|l}
x \in \mathbb{R}^{n} \backslash V(L) & \begin{array}{l}
x_{2 j-1}^{2}+x_{2 j}^{2}=\left(x_{2 j-1}^{0}\right)^{2}+\left(x_{2 j}^{0}\right)^{2} ; j=1, \cdots, p ; \\
x_{l}=x_{l}^{0}, ; l=2 p+1, \ldots, n
\end{array}
\end{array}\right\} .
$$

\footnotetext{
${ }^{3}$ Considere o sistema de EDO's definido por $\gamma^{\prime}(t)=L(\gamma(t))$. Para cada valor inicial $\gamma(0)=x$ o PVI admite uma única solução (teorema de Picard). Variando $x$ e considerando as soluções maximais de cada PVI obtemos uma partição de $\mathbb{R}^{n}$, que chamamos de folheação.
} 
Note que a propriedade (iv) requer que $V(L)$ tenha codimensão par, portanto, é imediato deste resultado que campos da forma (3.1.1) tais que $V(L)=\operatorname{ker}\left(m(L)^{\top}\right)$ tenha codimensão ímpar são localmente resolúveis em $V(L)$. Note ainda que como $L$ é invariante por translação em $V(L)$, temos que a propriedade $(i)$ é equivalente a $L$ não ser localmente resolúvel na origem. Finalmente, note que $(i v) \Rightarrow(i i)$, pois o toro é uma variedade compacta e, portanto, as órbitas contidas nele tem fecho compacto.

\subsection{Demonstração do Teorema}

\subsubsection{A equivalência de (ii), (iii) e (iv)}

Lema 3.3. As afirmações $(i i)$ e (iii) do Teorema 3.2 são equivalentes.

Demonstração: vamos mostrar primeiro que $(i i) \Rightarrow($ iii $)$. Para isso suponha que (iii) seja falso e seja $\chi=\xi+i \eta$ um autovalor de $m\left(L^{0}\right)$

Caso 1: $\eta=0$.

Como $\xi \in \mathbb{R}$ é um autovalor de $m\left(L^{0}\right)$, segue do Teorema da Forma Canônica de Jordan, que existe uma transformação linear tal que:

$$
m\left(L^{0}\right)=\left(\begin{array}{cc}
\xi & \overrightarrow{0} \\
\star & \star
\end{array}\right)
$$

e, portanto, podemos supor que $L$ é da forma:

$$
L=x_{1}\left(\xi \frac{\partial}{\partial x_{1}}+\sum_{k=m+1}^{n} \frac{\partial}{\partial x_{k}}\right)+\sum_{j=2}^{m} \sum_{k=1}^{n} a_{j k} x_{j} \frac{\partial}{\partial x_{k}}
$$

Observe que o campo $L$ na forma (3.2.1) é tangente à

$$
\dot{V}:=\left\{\vec{x} \in \mathbb{R}^{n} ; x_{1} \neq 0, x_{2}=\cdots=x_{m}=0\right\}^{4}
$$

e, portanto, aos semi-espaços $\dot{V}_{-}=\left\{x \in \dot{V} ; x_{1}<0\right\}$ e $\dot{V}_{+}=\left\{x \in \dot{V} ; x_{1}>0\right\}$. Assim,

\footnotetext{
${ }^{4}$ Lembrando que um campo $X: M \rightarrow T M$ em uma variedade $M$ é dito tangente à uma subvariedade $N \subseteq M$ quando $X(N) \subseteq T N \subseteq T M$. Em nosso caso, para $p \in \dot{V}$ o campo neste ponto é dado por $x_{1}\left(\bar{\xi} \partial_{1}+\sum_{k=m+1}^{n} a_{1 k} \partial_{k}\right)$. Como temos que $T_{p} \dot{V}=\left\{\vec{x} \in \mathbb{R}^{n} ; x_{2}=\cdots=x_{m}=0\right\}_{p}=\left[\partial_{1}, \partial_{m+1}, \ldots, \partial_{n}\right]$, para todo ponto $p \in \dot{V}$, segue que $L$ é tangente a $\dot{V}$
} 
temos que as $L$-órbitas coincidem ${ }^{5}$ com as órbitas do campo

$$
\left.L\right|_{\dot{V}_{ \pm}}=x_{1}\left(\xi \frac{\partial}{\partial x_{1}}+\sum_{k=m+1}^{n} a_{1 k} \frac{\partial}{\partial x_{k}}\right) .
$$

Neste caso, as órbitas por $x_{0} \in \dot{V}_{ \pm}$satisfazem:

$$
\left\{\begin{array}{l}
\dot{x}_{1}=\xi x_{1} \\
\dot{x}_{k}=a_{1 k} x_{1} \quad ; \quad k=m+1, \ldots, n \\
x(0)=x^{0}
\end{array}\right.
$$

Quando $\xi \neq 0$ a solução do P.V.I. acime é dada por

$$
x_{1}(t)=x_{1}^{0} e^{\xi t} ; x_{k}(t)=\frac{a_{1 k}}{\xi} x_{1}^{0} e^{\xi t}+x_{k}^{0}-\frac{a_{1 k}}{\xi} x_{1}^{0} \quad k=m+1, \ldots, n
$$

ou seja, são retas na direção do campo e, portanto, não tem fecho compacto. $\mathrm{O}$ caso em que $\xi=0$ tem solução dada por $x_{1}(t)=x_{1}^{0}$ e $x_{k}(t)=a_{1 k} x_{1}^{0} t+x_{k}^{0}$, que também é uma reta.

Neste último caso, poderíamos pensar sobre a possibilidade de que todos os $a_{1 k}$ sejam nulos, de modo que a órbita por $x_{0}$ seria um único ponto e portanto compacta. Mas é facil ver que isso não pode ocorrer, pois como já vimos, as únicas órbitas que são pontos são aquelas passando por $y_{0} \in V(L)$.

Caso 2: $\xi \neq 0 \neq \eta$.

Como $m\left(L^{0}\right)$ é uma matriz real, e $\xi+i \eta$ é um autovalor complexo, então a forma canônica de Jordan desta matriz pode ser tomada como:

$$
m\left(L^{0}\right)=\left(\begin{array}{ccc}
\xi & -\eta & \overrightarrow{0} \\
\eta & \xi & \overrightarrow{0} \\
\star & \star & \star
\end{array}\right)
$$

\footnotetext{
${ }^{5}$ Se um dado campo $X: M \rightarrow T M$ é tangente à uma subvariedade $N$, então dado um ponto $x_{0} \in N$, segue que a órbita que passa por este ponto está inteiramente contida em $N$.
} 
Assim, escrevendo $L$ da forma (3.1.3), temos

$$
\begin{aligned}
& L_{1}=\xi \frac{\partial}{\partial x_{1}}-\eta \frac{\partial}{\partial x_{2}}+\sum_{k=m+1}^{n} a_{1 k} \frac{\partial}{\partial x_{k}} \\
& L_{2}=\eta \frac{\partial}{\partial x_{1}}+\xi \frac{\partial}{\partial x_{2}}+\sum_{k=m+1}^{n} a_{2 k} \frac{\partial}{\partial x_{k}}
\end{aligned}
$$

Vamos fazer a mudança de variável ${ }^{6} \tilde{x}_{j}=x_{j}$ se $j=1, \cdots, m$ e

$$
\tilde{x}_{j}=x_{j}-\frac{1}{\xi^{2}+\eta^{2}}\left(\left(\xi a_{1 j}+\eta a_{2 j}\right) x_{1}+\left(-\eta a_{1 j}+\xi a_{2 j}\right) x_{2}\right)
$$

quando $j=m+1, \cdots, n$.

Claramente, para $3 \leq j \leq m$ temos que $L_{1}\left(\tilde{x}_{j}\right)=L_{2}\left(x_{j}\right)=0$. Além disso, observe que para $j>m$ temos

$$
\begin{aligned}
L_{1}\left(\tilde{x}_{j}\right) & =\xi \frac{\partial \tilde{x}_{j}}{\partial x_{1}}-\eta \frac{\partial \tilde{x}_{j}}{\partial x_{2}}+\sum_{k=m+1}^{n} a_{1 k} \frac{\partial \tilde{x}_{j}}{\partial x_{k}} \\
& =-\xi\left(\frac{\xi a_{1 j}+\eta a_{2 j}}{\xi^{2}+\eta^{2}}\right)-\eta\left(\frac{\eta a_{1 j}-\xi a_{2 j}}{\xi^{2}+\eta^{2}}\right)+a_{1 j}=0
\end{aligned}
$$

Finalmente, para $j=1$ ou $j=2$ temos $L_{1}\left(\tilde{x}_{1}\right)=\xi$ e $L_{1}\left(\tilde{x}_{2}\right)=-\eta$. Obtemos então

$$
\tilde{L}_{1}=\sum_{j=1}^{n} L_{1}\left(\tilde{x}_{j}\right) \frac{\partial}{\partial \tilde{x}_{j}}=\xi \frac{\partial}{\partial \tilde{x}_{1}}-\eta \frac{\partial}{\partial \tilde{x}_{2}}
$$

analogamente, obtemos:

$$
\tilde{L}_{2}=\eta \frac{\partial}{\partial \tilde{x}_{1}}+\xi \frac{\partial}{\partial \tilde{x}_{2}}
$$

${ }^{6}$ Lembrando que uma mudança de variável $\varphi: \mathbb{R}^{n} \rightarrow \mathbb{R}^{n}$ se manifesta no campo de vetores através do pushforward. Escrevendo $\varphi\left(x_{1}, \ldots, x_{n}\right)=\left(y_{1}, \ldots, y_{n}\right)$ e $L=\sum_{k=1}^{n} \sum_{j=1}^{m} a_{j k} x_{j} \frac{\partial}{\partial x_{k}}$, segue da definição do pushforward que

$$
\varphi_{*} L(y)=(d \varphi)_{x} L(x)=\left(\frac{\partial y_{k}}{\partial x_{l}}(x)\right)_{k, l}\left(\sum_{j=1}^{m} a_{j l} x_{j}\right)_{l}=\left(\sum_{l=1}^{n} \sum_{j=1}^{m} \frac{\partial y_{k}}{\partial x_{l}} a_{j l} x_{j}\right)_{k}=\left(L\left(y_{k}\right)\right)_{k}=\sum_{k=1}^{n} L\left(y_{k}\right) \frac{\partial}{\partial y_{k}}
$$


Neste novo sistema de coordenadas, fazendo $x_{j}=\tilde{x}_{j}$

$$
L=x_{1}\left(\xi \frac{\partial}{\partial x_{1}}-\eta \frac{\partial}{\partial x_{2}}\right)+x_{2}\left(\eta \frac{\partial}{\partial x_{1}}+\xi \frac{\partial}{\partial x_{2}}\right)+\sum_{j=3}^{m} x_{j} L_{j}
$$

Observe que o campo (3.2.2) é tangente ao espaço

$$
\dot{V}:=\left\{\vec{x} \in \mathbb{R}^{n} ; x_{1}^{2}+x_{2}^{2} \neq 0 ; x_{3}=\cdots x_{m}=0\right\}
$$

portanto,

$$
\left.L\right|_{\dot{V}}=x_{1}\left(\xi \frac{\partial}{\partial x_{1}}-\eta \frac{\partial}{\partial x_{2}}\right)+x_{2}\left(\eta \frac{\partial}{\partial x_{1}}+\xi \frac{\partial}{\partial x_{2}}\right) .
$$

Queremos agora passar para coordenadas polares $\left(x_{1}=r \cos \theta\right.$ e $\left.x_{2}=r \sin \theta\right)$. Note que em $\dot{V}$ esta mudança de coordenadas está bem definida pois retiramos a origem. Como já obervamos a forma correta de realizar a mudança de variáveis é através do pushforward. Neste caso, o difeomorfismo $\left(x_{1}, x_{2}\right) \mapsto(r, \theta)$ possui uma expressão não muito agradável, então utilizaremos o difeomorfismo inverso para explicitar a mudança de variáveis. Para facilitar a notação escreva

$$
\partial_{r}=\frac{\partial}{\partial r} \quad \partial_{\theta}=\frac{\partial}{\partial \theta} \quad \partial_{1}=\frac{\partial}{\partial x_{1}} \quad \partial_{2}=\frac{\partial}{\partial x_{2}}
$$

então,

$$
\left\{\begin{array}{l}
\partial_{r}=\left(\partial_{r} x_{1}\right) \partial_{1}+\left(\partial_{r} x_{2}\right) \partial_{2} \\
\partial_{\theta}=\left(\partial_{\theta} x_{1}\right) \partial_{1}+\left(\partial_{\theta} x_{2}\right) \partial_{2}
\end{array}\right.
$$

$\log \mathrm{O}$

$$
\left(\begin{array}{l}
\partial_{r} \\
\partial_{\theta}
\end{array}\right)=\left(\begin{array}{cc}
\cos \theta & \sin \theta \\
-r \sin \theta & r \cos \theta
\end{array}\right)\left(\begin{array}{l}
\partial_{1} \\
\partial_{2}
\end{array}\right)
$$

Invertendo a matriz obtemos

$$
\left(\begin{array}{l}
\partial_{1} \\
\partial_{2}
\end{array}\right)=\left(\begin{array}{cc}
\cos \theta & -\frac{1}{r} \sin \theta \\
\sin \theta & \frac{1}{r} \cos \theta
\end{array}\right)\left(\begin{array}{c}
\partial_{r} \\
\partial_{\theta}
\end{array}\right)
$$


daí

$$
\left\{\begin{array}{l}
\partial_{1}=\cos \theta \partial_{r}-\frac{1}{r} \sin \theta \partial_{\theta} \\
\partial_{2}=\sin \theta \partial_{r}+\frac{1}{r} \cos \theta \partial_{\theta}
\end{array}\right.
$$

Assim, obtemos

$$
\begin{aligned}
\left.L\right|_{\dot{V}}=r \cos \theta\left(\xi\left(\cos \theta \partial_{r}-\frac{1}{r} \sin \theta \partial_{\theta}\right)-\eta\left(\sin \theta \partial_{r}+\frac{1}{r} \cos \theta \partial_{\theta}\right)\right) \\
\quad+r \sin \theta\left(\eta\left(\cos \theta \partial_{r}-\frac{1}{r} \sin \theta \partial_{\theta}\right)+\xi\left(\sin \theta \partial_{r}+\frac{1}{r} \cos \theta \partial_{\theta}\right)\right) \\
=\xi r \frac{\partial}{\partial r}-\eta \frac{\partial}{\partial \theta}
\end{aligned}
$$

que tem como orbitas as espirais $\gamma(t)=(r, \theta)$, onde $r(\theta)=r_{0} \exp \left(-\eta^{-1} \xi \theta\right)$ e $\theta(t)=\eta t$, que não são limitadas e, portanto, não tem fecho compacto.

Caso 3: $\xi=0$ e a parte nilpotente de $m(L)$ não se anula.

Como já vimos, se $m\left(L^{0}\right)$ possui algum autovalor real, ou de parte real não nula, temos que existem $L$-órbitas ilimitadas e, portanto, de fecho não compacto. Vamos supor então que todos os autovalores de $m\left(L^{0}\right)$ são puramente imaginários. Logo, escrevendo $m(L)$ na forma canônica de Jordan, temos

$$
m(L)=\left(\begin{array}{cccccc}
B_{1} & 0 & & \cdots & & 0 \\
0 & \ddots & & & & \vdots \\
& & B_{l} & 0 & \cdots & 0 \\
\vdots & & 0 & 0 & \cdots & 0 \\
& & \vdots & \vdots & \ddots & \vdots \\
0 & \cdots & 0 & 0 & \cdots & 0
\end{array}\right)
$$

onde cada $B_{j}$ é um bloco de Jordan associado a um autovalores puramente imaginário $i \eta_{j}$ de multiplicidade algébrica $b_{j}$. Claramente temos

$$
m\left(L^{0}\right)=\left(\begin{array}{lll}
B_{1} & & \\
& \ddots & \\
& & B_{l}
\end{array}\right)
$$

Como a parte nilpotente de $m(L)$ não se anula, então existe um autovalor $i \eta$ com multiplicidade geométrica estritamente menor que a algébrica. Podemos supor, sem 
perda de generalidade que $\eta=\eta_{1}$ e, portanto, podemos escrever

$$
m(L)=\left(\begin{array}{ccccc}
0 & -\eta & 0 & 0 & \overrightarrow{0} \\
\eta & 0 & 0 & 0 & \overrightarrow{0} \\
1 & 0 & 0 & -\eta & \overrightarrow{0} \\
0 & 1 & \eta & 0 & \overrightarrow{0} \\
0 & 0 & \star & \star & \star
\end{array}\right)
$$

assim

$$
\begin{aligned}
L= & \eta\left(x_{1} \frac{\partial}{\partial x_{2}}-x_{2} \frac{\partial}{\partial x_{1}}+x_{3} \frac{\partial}{\partial x_{4}}-x_{4} \frac{\partial}{\partial x_{3}}\right) \\
& +x_{3} \frac{\partial}{\partial x_{1}}+x_{4} \frac{\partial}{\partial x_{2}}+\sum_{j=5}^{m} x_{j} L_{j}
\end{aligned}
$$

Observe que o campo na forma (3.2.4) é tangente à $\dot{V}=\left\{\vec{x} \in \mathbb{R}^{n} ; x_{1}^{2}+x_{2}^{2}+x_{3}^{2}+x_{4}^{2} \neq\right.$ $\left.0 ; x_{5}=\cdots=x_{m}=0\right\}$ e portanto

$$
\left.L\right|_{\dot{V}}=\left(-\eta x_{2}+x_{3}\right) \frac{\partial}{\partial x_{1}}+\left(\eta x_{1}+x_{4}\right) \frac{\partial}{\partial x_{2}}+\left(-\eta x_{4}\right) \frac{\partial}{\partial x_{3}}+\left(\eta x_{3}\right) \frac{\partial}{\partial x_{4}}
$$

Assim, a curva $\gamma$ em $\dot{V}$ parametrizada por

$$
x_{1}(t)=\frac{t}{\eta} \cos t ; \quad x_{2}(t)=\frac{t}{\eta} \sin t ; x_{3}(t)=\cos t ; x_{4}(t)=\sin t
$$

é tangente à $L$ e, portanto, obtemos novamente uma $L$-órbita de fecho não compacto.

Reciprocamente, vamos supor agora que (iii) seja satisfeita. Como todos os autovalores de $m\left(L^{0}\right)$ são puramente imaginários, podemos supor que $m(L)$ é da forma (3.2.3). Mas $m\left(L^{0}\right)$ tem parte nilpotente nula, logo, cada autovalor complexo de $m\left(L^{0}\right)$ tem multiplicidade algébrica igual à multiplicidade geométrica e assim, cada bloco de Jordan $B_{j}$ é da forma

$$
B_{j}=\left(\begin{array}{cc}
0 & -\eta_{j} \\
\eta_{j} & 0
\end{array}\right)
$$

com $j=0, \cdots, p$ e $m=2 p$ Nestas coordenadas temos:

$$
L^{0}=\sum_{j=1}^{p} \eta_{j}\left(x_{2 j-1} \frac{\partial}{\partial x_{2 j}}-x_{2 j} \frac{\partial}{\partial x_{2 j-1}}\right)
$$


portanto,

$$
\begin{aligned}
L & =\sum_{j=1}^{p} \eta_{j}\left(x_{2 j-1} \frac{\partial}{\partial x_{2 j}}-x_{2 j} \frac{\partial}{\partial x_{2 j-1}}\right)+\sum_{s=1}^{2 p} \sum_{l=m+1}^{n} x_{s} a_{s l} \frac{\partial}{\partial x_{l}} \\
& =\sum_{j=1}^{p} \eta_{j}\left(x_{2 j-1} \frac{\partial}{\partial x_{2 j}}-x_{2 j} \frac{\partial}{\partial x_{2 j-1}}\right)+\sum_{j=1}^{p} \sum_{l=m+1}^{n}\left(x_{2 j-1} a_{2 j-1, l}+x_{2 j} a_{2 j, l}\right) \frac{\partial}{\partial x_{l}} \\
& =\sum_{j=1}^{p} \eta_{j}\left(x_{2 j-1}\left(\frac{\partial}{\partial x_{2 j}}+\sum_{l=m+1}^{n} \frac{a_{2 j-1, l}}{\eta_{j}} \frac{\partial}{\partial x_{l}}\right)-x_{2 j}\left(\frac{\partial}{\partial x_{2 j-1}}-\sum_{l=m+1}^{n} \frac{a_{2 j, l}}{\eta_{j}} \frac{\partial}{\partial x_{l}}\right)\right) \\
& =\sum_{j=1}^{p} \eta_{j}\left(x_{2 j-1} L_{2 j}-x_{2 j} L_{2 j-1}\right)
\end{aligned}
$$

sendo

$$
\begin{aligned}
L_{k} & =\frac{\partial}{\partial x_{k}}+\sum_{l=2 p+1}^{n}(-1)^{k+1} \frac{1}{\eta_{\lfloor k / 2\rfloor}} a_{k l} \frac{\partial}{\partial x_{l}} \\
& =\frac{\partial}{\partial x_{k}}+\sum_{l=2 p+1}^{n} b_{k l} \frac{\partial}{\partial x_{l}} \quad k=1, \ldots, 2 p .
\end{aligned}
$$

A mudança de coordenadas

$$
\begin{cases}\tilde{x}_{k}=x_{k} & k=1, \ldots, 2 p \\ \tilde{x}_{j}=x_{j}-\sum_{l=2 p+1} b_{l j} x_{l} & j=2 p+1, \ldots, n\end{cases}
$$

transforma $L_{k}$ em $\tilde{L}_{k}=\frac{\partial}{\partial \tilde{x}_{k}}$ de modo que, após retirar o til, nas novas coordenadas, $L$ tem a forma:

$$
L=\sum_{j=1}^{p} \eta_{j}\left(x_{2 j-1} \frac{\partial}{\partial x_{2 j}}-x_{2 j} \frac{\partial}{\partial x_{2 j-1}}\right) .
$$

Fazendo a seguinte mudança de coordenadas

$$
x_{2 j-1}=r_{j} \cos \theta_{j} ; \quad x_{2 j}=r_{j} \sin \theta_{j} ; j=1, \cdots, p
$$

obtemos

$$
\tilde{L}=\sum_{j=1}^{p} \eta_{j} \frac{\partial}{\partial \theta_{j}}
$$


Para $x=\left(x_{1}, \ldots, x_{n}\right) \in \mathbb{R}^{n}$, denote por $|x|_{j}$ a seminorma

$$
|x|_{j}^{2}=x_{2 j-1}^{2}+x_{2 j}^{2} \quad ; \quad j=1, \ldots,\lfloor n / 2\rfloor^{7}
$$

Observe que a $L$-órbita passando por um ponto $q=\left(q_{1}, \cdots, q_{n}\right) \in \mathbb{R}^{n} \backslash V(L)$ é uma curva pelo toro

$$
(\tilde{\mathbb{T}})^{p}(q):=\left\{x \in \mathbb{R}^{n} \backslash V(L) ;|x|_{j}^{2}=|q|_{j}^{2} \text { e } x_{l}=q_{l} ; j=1, \ldots, p \text { e } l=2 p+1, \ldots, n\right\},
$$

pois $L$ não possui componentes radiais e, portanto, o fecho de uma $L$-órbitas em $\mathbb{R}^{n} \backslash V(L)$ é sempre compacto.

Corolário 3.4. Se o div $L \neq 0$, então existe uma $L$-órbita em $\mathbb{R}^{n} \backslash V(L)$ cujo fecho não é compacto em $\mathbb{R}^{n} \backslash V(L)$

Demonstração: Basta observar que se div $L \neq 0$ então existe um autovalor de $m\left(L^{0}\right)$ com parte real não nula.

Observação 3.5. Note que, se $\left(M_{1}, g_{1}\right)$ e $\left(M_{2}, g_{2}\right)$ são variedades Riemannianas e $\gamma_{j}$ : $I \rightarrow M_{j}, j=1,2$, são geodesicas, então $\left(\gamma_{1}, \gamma_{2}\right): I \rightarrow M_{1} \times M_{2}$ é uma geodésica ${ }^{8}$ de $\left(M_{1} \times M_{2}, g_{1}+g_{2}\right)$. Assim, se considerarmos o toro (3.2.8) como um toro flat, isto é, como o produto cartesiano (entre variedades riemannianas com a métrica produto) de diversas circunferências, podemos afirmar que a $L$-órbita passando pelo ponto $q$ definida pelo campo (3.2.7) é na verdade uma geodésica deste toro, pois essas $L$-órbitas são da forma $\left(\gamma_{1}, \cdots, \gamma_{m}\right)$, onde cada $\gamma_{j}$ é uma geodésica do círculo ${ }^{9}$ em $\mathbb{R}^{n}$ dado por:

$$
x_{l}=q_{l}, l=1, \ldots, 2 j-2,2 j+1, \ldots, n \quad x_{2 j-1}^{2}+x_{2 j}^{2}=|q|_{j}^{2}
$$

Segue do Lema 3.3 e da observação acima que (ii), (iii) e (iv) são equivalentes (é trivial que $(i v)$ implica $(i i))$.

\footnotetext{
${ }^{7}$ Aqui, $\lfloor x\rfloor$ denota o maior inteiro $M$ tal que $M \leq x$

${ }^{8}$ Ver apêndice $\mathrm{A}$.

${ }^{9}$ Lembrando que as geodésicas do círculo são arcos do círculo parametrizados com velocidade constante.
} 


\subsubsection{O caso $\operatorname{dim} V(L)=n-1$}

Nesta subseção, vamos demonstrar que o teorema é verdadeiro se $m=\operatorname{codim} V(L)=1$. Em particular, observe que $m$ é ímpar, e, portanto, das considerações feitas anteriormente, isso deve implicar que $L$ é localmente resolúvel.

Lema 3.6. Se $X$ é um campo de vetores com coeficientes (não todos nulos) constantes e, se $h$ é uma função analítica em um aberto convexo $\Omega \subset \mathbb{R}^{n}$, então o campo $h(x) X$ é localmente resolúvel em $\Omega$.

Demonstração: Um "famoso" resultado provado por Lojasiewics [1959] afirma que é possível dividir uma distribuição arbitrária por uma função analítica, isto é, dada $h$ analítica e $v \in \mathcal{D}^{\prime}(\Omega)$, então sempre existe solução $u \in \mathcal{D}^{\prime}(\Omega)$ para o problema $h(x) u=v$. Assim, resolver o problema $h(x) X u=f, f \in \mathcal{D}^{\prime}(\Omega)$, é equivalente a resolver o problema $X u=h^{-1} f$. O teorema de Malgrange-Ehrempreis não é aplicável aqui, pois $h^{-1} f$ não é necessariamente uma função suave de suporte compacto. Precisamos então recorrer ao resultado do Hörmander (Teorema 2.3). Este resultado garante que $X$ é localmente resolúvel em todos os pontos, basta tomar um aberto convexo em torno de cada ponto.

Sabemos que no caso $\operatorname{dim} V(L)=n-1$, o campo (3.1.1) é da forma

$$
L=x_{1} L_{1} \quad ; \quad L_{1}=\sum_{k=1}^{n} a_{k} \frac{\partial}{\partial x_{k}}
$$

como $h(x)=x_{1}$ é analítica e $L_{1}$ tem coeficientes constantes, segue do lema anterior que $L$ é localmente resolúvel. Note que as órbitas por $x^{0}=\left(x_{1}^{0}, \ldots, x_{n}^{0}\right) \in \mathbb{R}^{n} \backslash V(L)$ satisfazem o seguinte sistema de EDOs.

$$
\left\{\begin{array}{l}
\dot{x}_{k}=a_{k} x_{1} \quad ; \quad k=1, \ldots, n \\
x(0)=x^{0}
\end{array}\right.
$$

daí, temos dois casos:

Caso 1: $a_{1} \neq 0$.

Neste caso, $x_{1}(t)=x_{1}^{0} e^{a_{1} t}$ e $x_{k}(t)=\frac{a_{k}}{a_{1}} x_{1}^{0} e^{a_{1} t}+x_{k}^{0}-\frac{a_{k}}{a_{1}} x_{1}^{0}$ e, portanto, as órbitas por $x^{0}$ são retas e, portanto, não tem fecho compacto.

Caso 2: $a_{1}=0$. 
Neste caso $x_{1}(t)=x_{1}^{0}$ e $x_{k}(t)=a_{k} x_{1}^{0} t+x_{k}^{0}$. Note que existe $a_{k} \neq 0$ pois do contrário, o campo seria identicamente nulo. Logo, como no caso anterior, as órbitas são retas.

Assim, no caso $m=\operatorname{codim} V(L)=1$, temos que o campo $L$ é localmente resolúvel, existem $L$-órbitas com fecho não compacto e, portanto, não é possível que exista um sistema de coordenadas em que todas as $L$-órbitas sejam geodésicas de um toro. Provamos assim, que as afirmações $(i),(i i)$ e $(i v)$ do teorema são válidas no caso em que $m=1$. A equivalência entre as afirmações $(i i)$ e $($ iii $)$ finaliza a demonstração para o caso $m=1$.

\subsubsection{O caso $m=\operatorname{codim} V(L) \geq 2$}

Observe que o Lema 3.3 demonstrado anteriormente, seu subsequente corolário e observação são válidos para quaisquer valor de $m=\operatorname{codim} V(L)$. Assim, as afirmações (ii), (iii) e (iv) do Teorema 3.2 são equivalentes. Resta então mostrar que $(i)$ é equivalente a $($ ii) (ou a $(i i i)$ ) quando $m \geq 2$. Observe ainda que como $L$ é invariante por translação em $V(L)$, temos que a propriedade (i) é equivalente à $L$ não ser localmente resolúvel na origem.

No que se segue, $\langle\cdot, \cdot\rangle_{L^{2}}$ e $\|\cdot\|_{L^{2}}$ denotam o produto interno e a norma em $L^{2}(\Omega)$ respectivamente.

Lema 3.7. Seja $\Omega \subset \mathbb{R}^{n}$ aberto. Suponha que exista um operador diferencial parcial linear com coeficientes constantes $P(\partial)$ tal que, para algum $C>0$ e para todo $\varphi \in C_{c}^{\infty}(\Omega)$

$$
\|\varphi\|_{L^{2}} \leq C\left\|P(\partial) L^{\top} \varphi\right\|_{L^{2}}
$$

Então $L^{2}(\Omega) \subset L \mathcal{D}^{\prime}(\Omega)$. Mais precisamente, existe uma aplicação contínua $\tilde{E}$ : $L^{2}(\Omega) \rightarrow L^{2}(\Omega)$ tal que

$$
L P(\partial) \tilde{E}^{\top} f=f, \quad \forall f \in L^{2}(\Omega)
$$

Demonstração: Segue da estimativa (3.2.9) que a aplicação

$$
\begin{gathered}
E: P(\partial) L^{\top} C_{c}^{\infty}(\Omega) \rightarrow C_{c}^{\infty}(\Omega) \\
P(\partial) L^{\top} \varphi \mapsto \varphi
\end{gathered}
$$

está bem definida ${ }^{10}$ e se estende (pelo teorema de Hahn-Banach) a uma aplicação linear

\footnotetext{
${ }^{10} \mathrm{Se} P(\partial) L^{\top} \varphi=P(\partial) L^{\top} \psi$, então $\|\varphi-\psi\| \leq C\left\|P(\partial) L^{\top}(\varphi-\psi)\right\|=0$, daí, $\varphi=\psi$, exceto em um conjunto de medida nula, mas como são contínuas, segue que $\varphi=\psi$.
} 
contínua $\tilde{E}: L^{2}(\Omega) \rightarrow L^{2}(\Omega)$ com a propriedade de que $\tilde{E} P(\partial) L^{\top} \varphi=\varphi$ para toda $\varphi \in C_{c}^{\infty}(\Omega)$. Assim:

$$
\begin{aligned}
\left\langle L P(-\partial) \tilde{E}^{\top} f, \varphi\right\rangle & =\left\langle P(-\partial) \tilde{E}^{\top} f, L^{\top} \varphi\right\rangle=\left\langle\tilde{E}^{\top} f, P(\partial) L^{\top} \varphi\right\rangle \\
& =\left\langle f, \tilde{E} P(\partial) L^{\top} \varphi\right\rangle=\langle f, \varphi\rangle
\end{aligned}
$$

e, portanto, $L P(-\partial) \tilde{E}^{\top} f=f$ para toda $f \in L^{2}(\Omega)^{11}$. Em particular, $L$ é localmente resolúvel em $\Omega$.

Lema 3.8. Seja $\varphi$ função suave de suporte compacto. Então

$$
2 \mathfrak{R e}\left\langle L^{\top} \varphi, \varphi\right\rangle_{L^{2}}=-(\operatorname{div} L)\|\varphi\|_{L^{2}}^{2}
$$

Demonstração: Seja $\varphi \in C_{c}^{\infty}$, então

$$
\begin{aligned}
\left\langle L^{\top} \varphi, \varphi\right\rangle_{L^{2}} & =\langle\varphi, L \varphi\rangle_{L^{2}}=\int(L \varphi) \bar{\varphi} d x \\
& =\int \sum_{j=1}^{m} \sum_{k=1}^{n} a_{j k} x_{j} \frac{\partial \varphi}{\partial x^{k}}(x) \bar{\varphi}(x) d x=\sum_{j=1}^{m} \sum_{k=1}^{n} a_{j k} \int x_{j} \frac{\partial \varphi}{\partial x^{k}}(x) \bar{\varphi}(x) d x .
\end{aligned}
$$

Note que se $j \neq k$ temos

$$
\begin{aligned}
\mathcal{I} & \doteq \int x_{j} \frac{\partial \varphi}{\partial x_{k}}(x) \bar{\varphi}(x) d x_{k}=x_{j} \int \frac{\partial \varphi}{\partial x_{k}}(x) \bar{\varphi}(x) d x_{k} \\
& =-x_{j} \int \varphi(x) \frac{\partial \bar{\varphi}}{\partial x_{k}}(x) d x_{k}=-\overline{\mathcal{I}}
\end{aligned}
$$

e, portanto, $\mathfrak{R e}(\mathcal{I})=0$.

No caso em que $j=k$ temos:

$$
\begin{aligned}
\mathcal{J} & \doteq \int x_{j} \frac{\partial \varphi}{\partial x_{j}}(x) \bar{\varphi}(x) d x_{j}=-\int \varphi\left(\bar{\varphi}+x_{j} \frac{\partial \bar{\varphi}}{\partial x_{j}}\right) d x_{j} \\
& =-\int \varphi(x) \bar{\varphi}(x) d x_{j}-\overline{\mathcal{J}}
\end{aligned}
$$

${ }^{11}$ Onde a igualdade em questão é no sentido das distribuições 
e $\operatorname{assim} 2 \mathfrak{R e}(\mathcal{J})=-\int|\varphi|^{2} d x_{j}$. Segue então que:

$$
\begin{array}{r}
2 \mathfrak{R e}\left\langle L^{\top} \varphi, \varphi\right\rangle_{L^{2}}=\sum_{j=1}^{m} 2 a_{j j} \mathfrak{R e} \int x_{j} \frac{\partial \varphi}{\partial x^{j}}(x) \bar{\varphi}(x) d x \\
=-\sum_{j=1}^{m} a_{j j} \int|\varphi|^{2} d x=-(\operatorname{div} L)\|\varphi\|_{L^{2}}^{2}
\end{array}
$$

Corolário 3.9. Seja $L$ o campo de vetores (3.1.2). Se div $L \neq 0$ então $L$ é localmente resolúvel.

Demonstração: Aplicando a desigualdade de Cauchy-Schwarz ao Lema 3.8 obtemos:

$$
|\operatorname{div} L|\|\varphi\|_{L^{2}}^{2}=\left|2 \mathfrak{R e}\left\langle L^{\top} \varphi, \varphi\right\rangle_{L^{2}}\right| \leq 2\left\|L^{\top} \varphi\right\|_{L^{2}}\|\varphi\|_{L^{2}}
$$

Segue então do Lema 3.7 que existe aplicação linear contínua $E: L^{2}\left(\mathbb{R}^{n}\right) \rightarrow L^{2}\left(\mathbb{R}^{n}\right)$ tal que $L E^{\top} f=f$ para toda $f \in L^{2}\left(\mathbb{R}^{n}\right)$.

O próximo passo da demonstração do Teorema 3.2 é provar que se os autovalores de $m\left(L^{0}\right)$ tem parte real não nula ou $m\left(L^{0}\right)$ tem parte nilpotente não nula, então $L$ é localmente resolúvel. Para concluirmos isso, iremos obter desigualdades do tipo (3.2.9) e aplicaremos o Lema 3.7. Neste contexto, os lemas a seguir serão úteis para se obter estas desigualdades.

Lema 3.10. Sejam $P(D)$ uma derivada direcional na direção $v$ e $[v] \subset \mathbb{R}^{n}$ o subespaço linear gerado por $v$. Seja ainda $W$ o espaço ortogonal a $[v]$ e defina $B_{R} \subset \mathbb{R}^{n}$ dado por

$$
B_{R}=\left\{x=\lambda v \oplus y \in[v] \oplus W=\mathbb{R}^{n} ; \lambda \in \mathbb{R} ;|\lambda|<\frac{R}{2}\right\}
$$

Então, para cada $R \in \mathbb{R}_{+}$, existe constante $C_{R}$ tal que

$$
\|\varphi\|_{L^{2}} \leq C_{R}\|P(D) \varphi\|_{L^{2}} \quad \forall \varphi \in C_{c}^{\infty}\left(B_{R}\right)
$$

Mais precisamente, basta tomar $C_{R}=2 R$. 
Demonstração: Segue do teorema fundamental do cálculo que

$$
\varphi(x)-\varphi(x-R v)=\int_{-R}^{0} \frac{d}{d s}[\varphi(x+s v)] d s=\int_{-R}^{0} P(D) \varphi(x+s v) d s
$$

assim, segue do Lema B.14

$$
\begin{aligned}
\|\varphi(\cdot)-\varphi(\cdot-R v)\|_{L^{2}} & =\left\|\int_{-R}^{0} P(D) \varphi(x+s v) d s\right\|_{L_{2}} \leq \int_{-R}^{0}\|P(D) \varphi(x+s v)\|_{L^{2}} d s \\
& =\int_{-R}^{0}\|P(D) \varphi\|_{L^{2}} d s=R\|P(D) \varphi\|_{L^{2}},
\end{aligned}
$$

onde a norma $L^{2}$ acima é com respeito à variável $x$. Note que, se $\varphi$ tem suporte em $B_{R}$, então, $\varphi(x-R v)=0$ para todo $x \in B_{R}$ e, portanto, fixando o suporte de $\varphi$ obtemos:

$$
\|\varphi\|_{L^{2}}=\|\varphi(\cdot)-\varphi(\cdot-R v)\|_{L^{2}} \leq R\|P(D) \varphi\|_{L^{2}}, \forall \varphi \in C_{c}^{\infty}\left(B_{R}\right)
$$

Lema 3.11. Seja uma vizinhança da origem $\Omega \subset \mathbb{R}^{n}, n \geq 2$ e denotemos por $\bar{\partial}$ o seguinte operador nas duas primeiras variáveis

$$
\bar{\partial} \varphi \doteq \frac{\partial \varphi}{\partial x_{1}}+i \frac{\partial \varphi}{\partial x_{2}}
$$

Para cada $R>0$ defina $\tilde{B}_{R} \doteq\left\{x \in \Omega ; \max \left\{\left|x_{1}\right|,\left|x_{2}\right|\right\}<R / 2\right\}$ então, existe $C_{R}>0$ tal que:

$$
\|\varphi\|_{L^{2}} \leq C_{R}\|\bar{\partial} \varphi\|_{L^{2}} ; \forall \varphi \in C_{c}^{\infty}\left(\tilde{B}_{R}\right)
$$

Demonstração: Seja $\varphi=\varphi_{1}+i \varphi_{2} \in C_{c}^{\infty}\left(\tilde{B}_{R}\right)$. Temos que

$$
\frac{\partial \varphi}{\partial x_{1}}=\frac{\partial \varphi_{1}}{\partial x_{1}}+i \frac{\partial \varphi_{2}}{\partial x_{1}} ; \frac{\partial \varphi}{\partial x_{2}}=\frac{\partial \varphi_{1}}{\partial x_{2}}+i \frac{\partial \varphi_{2}}{\partial x_{2}}
$$

assim, $\bar{\partial} \varphi=\frac{\partial \varphi_{1}}{\partial x_{1}}-\frac{\partial \varphi_{2}}{\partial x_{2}}+i \frac{\partial \varphi_{2}}{\partial x_{1}}+i \frac{\partial \varphi_{1}}{\partial x_{2}}$ e, portanto,

$$
\begin{aligned}
|\bar{\partial} \varphi|^{2}= & \left(\frac{\partial \varphi_{1}}{\partial x_{1}}\right)^{2}+\left(\frac{\partial \varphi_{2}}{\partial x_{2}}\right)^{2}+\left(\frac{\partial \varphi_{2}}{\partial x_{1}}\right)^{2}+\left(\frac{\partial \varphi_{1}}{\partial x_{2}}\right)^{2}-2\left(\frac{\partial \varphi_{1}}{\partial x_{1}} \frac{\partial \varphi_{2}}{\partial x_{2}}-\frac{\partial \varphi_{2}}{\partial x_{1}} \frac{\partial \varphi_{1}}{\partial x_{2}}\right) \\
& =\left|\frac{\partial \varphi}{\partial x_{1}}\right|^{2}+\left|\frac{\partial \varphi}{\partial x_{2}}\right|^{2}-2\left(\frac{\partial \varphi_{1}}{\partial x_{1}} \frac{\partial \varphi_{2}}{\partial x_{2}}-\frac{\partial \varphi_{2}}{\partial x_{1}} \frac{\partial \varphi_{1}}{\partial x_{2}}\right)
\end{aligned}
$$


integrando por partes, temos que

$$
\int_{B_{R}} \frac{\partial \varphi_{1}}{\partial x_{1}} \frac{\partial \varphi_{2}}{\partial x_{2}}-\frac{\partial \varphi_{2}}{\partial x_{1}} \frac{\partial \varphi_{1}}{\partial x_{2}} d x=0
$$

e assim

$$
\|\bar{\partial} \varphi\|_{L^{2}}^{2}=\left\|\frac{\partial \varphi}{\partial x_{1}}\right\|_{L^{2}}^{2}+\left\|\frac{\partial \varphi}{\partial x_{2}}\right\|_{L^{2}}^{2}
$$

Basta agora aplicar o Lema 3.10 à cada uma destas derivadas direcionais, e obtemos

$$
\|\bar{\partial} \varphi\|_{L^{2}}^{2}=\left\|\frac{\partial \varphi}{\partial x_{1}}\right\|_{L^{2}}^{2}+\left\|\frac{\partial \varphi}{\partial x_{2}}\right\|_{L^{2}}^{2} \geq \frac{1}{R^{2}}\|\varphi\|^{2}+\frac{1}{R^{2}}\|\varphi\|^{2}=\frac{2}{R^{2}}\|\varphi\|^{2}
$$

para toda $\varphi \in \tilde{B}_{R}$, de modo que

$$
\|\varphi\| \leq R \sqrt{2} / 2\|\bar{\partial} \varphi\| \quad \forall \varphi \in \tilde{B}_{R}
$$

como queríamos demonstrar.

Como já verificamos o caso $m=1$, vamos supor, daqui em diante, que $m \geq 2$. Iremos primeiramente mostrar que $(i) \Rightarrow($ iii $)$, isto é, mostrar que se $m\left(L^{0}\right)$ possui autovalores com parte real não nula ou $m\left(L^{0}\right)$ tem parte nilpotente não nula, então $L$ é localmente resolúvel em $V(L)$. O caso em que div $L \neq 0$ segue do Corolário 3.9. Portanto, no que se segue vamos nos restingir ao caso em que $\operatorname{div} L=0$,

Lema 3.12. Seja $L$ o campo de vetores (3.1.2). Suponha que $\operatorname{div} L=0$. Então $L^{T}=-L$.

Demonstração: Se $u \in \mathcal{D}^{\prime}(\Omega)$ e $\varphi \in C_{c}^{\infty}$, então:

$$
\begin{aligned}
\left\langle L^{\top} u+L u, \varphi\right\rangle & =\langle u, L \varphi\rangle+\langle L u, \varphi\rangle=\left\langle u, \sum_{j, k} a_{j k} x_{j} \partial_{k} \varphi\right\rangle+\left\langle\sum_{j, k} a_{j k} x_{j} \partial_{k} u, \sum_{j, k} a_{j k} x_{j} \partial_{k} \varphi\right\rangle \\
& =\sum_{j, k} a_{j k}\left(\left\langle u, x_{j} \partial_{k} \varphi\right\rangle+\left\langle x_{j} \partial_{k} u, \varphi\right\rangle\right)=\sum_{j, k} a_{j k}\left(\left\langle u, x_{j} \partial_{k} \varphi\right\rangle-\left\langle u, \partial_{k}\left(x_{j} \varphi\right)\right\rangle\right) \\
& =\sum_{j=k} a_{j j}\left(\left\langle u, x_{j} \partial_{j} \varphi\right\rangle-\left\langle u, \varphi+\partial_{k} \varphi\right\rangle\right)=\sum_{j=k} a_{j j}\left\langle u, \partial_{j}\right\rangle=\operatorname{div}(L)\langle u, \varphi\rangle=0 .
\end{aligned}
$$

Proposição 3.13. Seja $L$ um campo de vetores da forma (3.1.2). Suponha que div $L=0$ e que $m\left(L^{0}\right)$ possua um autovalor $\xi+\eta i \operatorname{com} \xi \neq 0 \neq \eta$. Então $L$ é localmente resolúvel em $\mathbb{R}^{n}$. 
Demonstração: Como já vimos, nas condições acima o campo $L$ pode ser assumido como da forma (3.2.2). Derivando com respeito à $x_{1}$ e $x_{2}$ separadamente obtemos:

$$
\begin{aligned}
& \frac{\partial}{\partial x_{1}} L=L \frac{\partial}{\partial x_{1}}+\xi \frac{\partial}{\partial x_{1}}-\eta \frac{\partial}{\partial x_{2}} \\
& \frac{\partial}{\partial x_{2}} L=L \frac{\partial}{\partial x_{2}}+\eta \frac{\partial}{\partial x_{1}}-\xi \frac{\partial}{\partial x_{2}} .
\end{aligned}
$$

Multiplicando a segunda equação por $i$ e somando as duas equações obtemos:

$$
\left(\frac{\partial}{\partial x_{1}}+i \frac{\partial}{\partial x_{2}}\right) L=L\left(\frac{\partial}{\partial x_{1}}+i \frac{\partial}{\partial x_{2}}\right)+(\xi+\eta i)\left(\frac{\partial}{\partial x_{1}}+i \frac{\partial}{\partial x_{2}}\right) .
$$

Denotemos por $\tilde{P}(\partial)=\frac{\partial}{\partial x_{1}}+i \frac{\partial}{\partial x_{2}}$. Temos assim que

$$
\tilde{P}(\partial)(L \varphi)=L \tilde{P}(\partial) \varphi+(\xi+\eta i) \tilde{P}(\partial) \varphi
$$

para qualquer $\varphi \in C_{c}^{\infty}\left(\mathbb{R}^{n}\right)$. Temos ainda que

$$
\mathfrak{R e}\langle L \tilde{P}(\partial) \varphi+(\xi+\eta i) \tilde{P}(\partial) \varphi, \tilde{P}(\partial) \varphi\rangle_{L^{2}}=\xi\|\tilde{P}(\partial) \varphi\|_{L^{2}}^{2}
$$

e, portanto,

$$
\mathfrak{R e}\left\langle(\tilde{P}(\partial))^{2} L \varphi, \varphi\right\rangle_{L^{2}}=\xi\|\tilde{P}(\partial) \varphi\|_{L^{2}}^{2}
$$

Aplicando o Lema 3.11 no lado direito da equação acima e a desigualdade de Cauchy-Riemann no lado esquerdo da identidade obtemos uma desigualdade que atende as hipóteses do Lema 3.7 obtendo então o resultado desejado.

Proposição 3.14. Seja $L$ o campo de vetores (3.1.1) com $\operatorname{div} L=0$ e suponha que $m\left(L^{0}\right)$ possui um autovalor real $\xi \neq 0$, então $L$ é localmente resolúvel em $\mathbb{R}^{n}$.

Demonstração: Observe que nas condições do teorema, podemos supor que $L$ é da forma (3.2.1). Definindo, então, o operador $P(D)$ por

$$
P(D)=\xi \frac{\partial}{\partial x_{1}}+\sum_{k=m+1}^{n} a_{1 k} \frac{\partial}{\partial x_{k}}
$$

temos que $P(D) L=(L+\xi) P(D)$. Lembrando que div $L=0$, temos

$$
\mathfrak{R e}\langle(L+\xi) \varphi, \varphi\rangle_{L^{2}}=-\left\langle\left(L^{T} \varphi, \varphi\right\rangle_{L^{2}}+\xi\langle\varphi, \varphi\rangle_{L^{2}}=\xi\|\varphi\|_{L^{2}}^{2} .\right.
$$


assim,

$$
\mathfrak{R e}\left\langle P(D)^{2} L \varphi, \varphi\right\rangle_{L^{2}}=-\mathfrak{R e}\langle(L+\xi) P(D) \varphi, P(D) \varphi\rangle_{L^{2}}=-\xi\|P(D) \varphi\|_{L^{2}}^{2} .
$$

Aplicando o Lema 3.10, obtemos:

$$
\begin{aligned}
\|\varphi\|_{L^{2}}^{2} & \leq n^{2}\|P(D) \varphi\|_{L^{2}}^{2} \leq n^{2} \mathfrak{R e}\left\langle P(D)^{2} L \varphi, \varphi\right\rangle_{L^{2}} \\
& \leq n^{2}\left\|\left\langle P(D)^{2} L \varphi, \varphi\right\rangle_{L^{2}}\right\|_{L^{2}} \leq n^{2}\left\|P(D)^{2} L \varphi\right\|_{L^{2}}\|\varphi\|_{L^{2}}
\end{aligned}
$$

e, portanto, $\|\varphi\|<C\left\|P(D)^{2} L \varphi\right\|$ para qualquer $\varphi$ com suporte em $B_{n / 2}(0)$. O resultado segue então do Lema 3.7.

Proposição 3.15. Seja $L$ o campo de vetores (3.1.1) com div $L=0$ e suponha que $m\left(L^{0}\right)$ possui um autovalor nulo, então $L$ é localmente resolúvel em $\mathbb{R}^{n}$.

Demonstração: Como já foi visto anteriormente, nas condições da proposição o campo é da forma:

$$
L=x_{1} \sum_{k=m+1}^{n} a_{1 k} \frac{\partial}{\partial x_{k}}+\sum_{j=2}^{m} \sum_{k=1}^{n} a_{j k} x_{j} \frac{\partial}{\partial x_{k}} .
$$

Denote $P(D)=\sum_{k=m+1}^{n} a_{1 k} \frac{\partial}{\partial x_{k}}$. Note que $P(D)^{T}=-P(D)$. Além disso,

$$
\begin{aligned}
\frac{\partial}{\partial x_{1}} L \varphi & =P(D) \varphi+L \frac{\partial \varphi}{\partial x_{1}} \\
P(D)(L \varphi) & =L(P(D) \varphi) .
\end{aligned}
$$

Daí,

$$
\begin{aligned}
\left\langle L \varphi, P(D) \frac{\partial \varphi}{\partial x_{1}}\right\rangle_{L^{2}} & =-\left\langle L P(D) \varphi, \frac{\partial \varphi}{\partial x_{1}}\right\rangle_{L^{2}}=\left\langle\frac{\partial}{\partial x_{1}}(L P(D) \varphi), \varphi\right\rangle_{L^{2}} \\
& =\left\langle P(D)^{2} \varphi+L P(D) \frac{\partial \varphi}{\partial x_{1}}, \varphi\right\rangle_{L^{2}} \\
& =\left\langle P(D)^{2} \varphi, \varphi\right\rangle_{L^{2}}+\left\langle L P(D) \frac{\partial \varphi}{\partial x_{1}}, \varphi\right\rangle_{L^{2}} \\
& =-\|P(D) \varphi\|_{L^{2}}^{2}-\left\langle P(D) \frac{\partial \varphi}{\partial x_{1}}, L \varphi\right\rangle_{L^{2}} .
\end{aligned}
$$


Portanto:

$$
2 \mathfrak{R e}\left(\left\langle L \varphi, P(D) \frac{\partial \varphi}{\partial x_{1}}\right\rangle_{L^{2}}\right)=\left\langle L \varphi, P(D) \frac{\partial \varphi}{\partial x_{1}}\right\rangle_{L^{2}}+\overline{\left\langle L \varphi, P(D) \frac{\partial \varphi}{\partial x_{1}}\right\rangle_{L^{2}}}=-\|P(D) \varphi\|_{L^{2}}^{2}
$$

Podemos então proceder como na demonstração anterior e concluir que $L$ é localmente resolúvel.

Resta agora considerarmos o caso em que todos os autovalores não nulos de $m(L)$ são puramente imaginários.

Proposição 3.16. Seja $L$ um campo de vetores tal que todos os autovalores de $m\left(L^{0}\right)$ sejam puramente imaginários. Suponha ainda que a parte nilpotente de $m\left(L^{0}\right)$ não seja identicamente nula. Então $L$ é localmente resolúvel em todos os pontos de $V(L)$.

Demonstração: Como já vimos, nas condições acima podemos supor, sem perda de generalidade, que a matriz $m(L)$ é da forma

$$
m(L)=\left(\begin{array}{ccccc}
0 & -\eta & 0 & 0 & \overrightarrow{0} \\
\eta & 0 & 0 & 0 & \overrightarrow{0} \\
1 & 0 & 0 & -\eta & \overrightarrow{0} \\
0 & 1 & \eta & 0 & \overrightarrow{0} \\
0 & 0 & \star & \star & \star
\end{array}\right)
$$

e, portanto, o campo $L$ é dado por:

$$
\begin{aligned}
L= & \eta\left(x_{1} \frac{\partial}{\partial x_{2}}-x_{2} \frac{\partial}{\partial x_{1}}+x_{3} \frac{\partial}{\partial x_{4}}-x_{4} \frac{\partial}{\partial x_{3}}\right) \\
& +x_{3} \frac{\partial}{\partial x_{1}}+x_{4} \frac{\partial}{\partial x_{2}}+\sum_{j=5}^{m} x_{j} L_{j} .
\end{aligned}
$$

Derivando em relação às quatro primeiras variáveis obtemos:

$$
\begin{aligned}
& \frac{\partial}{\partial x_{1}}(L \varphi)=L \frac{\partial \varphi}{\partial x_{1}}+\eta \frac{\partial \varphi}{\partial x_{2}} \\
& \frac{\partial}{\partial x_{2}}(L \varphi)=L \frac{\partial \varphi}{\partial x_{2}}-\eta \frac{\partial \varphi}{\partial x_{1}} \\
& \frac{\partial}{\partial x_{3}}(L \varphi)=L \frac{\partial \varphi}{\partial x_{3}}+\eta \frac{\partial \varphi}{\partial x_{4}}+\frac{\partial \varphi}{\partial x_{1}} \\
& \frac{\partial}{\partial x_{4}}(L \varphi)=L \frac{\partial \varphi}{\partial x_{4}}-\eta \frac{\partial \varphi}{\partial x_{3}}+\frac{\partial \varphi}{\partial x_{2}}
\end{aligned}
$$


Das propriedades do produto interno em $L^{2}\left(\mathbb{R}^{n}\right)^{12}$ obtemos que:

$$
\begin{aligned}
\left\langle\frac{\partial}{\partial x_{3}}(L \varphi), \frac{\partial}{\partial x_{1}} \varphi\right\rangle_{L^{2}} & =\left\langle L\left(\frac{\partial}{\partial x_{3}} \varphi\right), \frac{\partial}{\partial x_{1}} \varphi\right\rangle_{L^{2}}+\eta\left\langle\frac{\partial}{\partial x_{4}} \varphi, \frac{\partial}{\partial x_{1}} \varphi\right\rangle_{L^{2}}+\left\|\frac{\partial}{\partial x_{1}} \varphi\right\|_{L^{2}}^{2} \\
& =-\left\langle\frac{\partial}{\partial x_{3}} \varphi, L\left(\frac{\partial}{\partial x_{1}} \varphi\right)\right\rangle_{L^{2}}+\eta\left\langle\frac{\partial}{\partial x_{4}} \varphi, \frac{\partial}{\partial x_{1}} \varphi\right\rangle_{L^{2}}+\left\|\frac{\partial}{\partial x_{1}} \varphi\right\|_{L^{2}}^{2} \\
& =-\left(\left\langle\frac{\partial}{\partial x_{3}} \varphi, \frac{\partial}{\partial x_{1}}(L \varphi)\right\rangle_{L^{2}}-\eta\left\langle\frac{\partial}{\partial x_{3}} \varphi, \frac{\partial}{\partial x_{2}} \varphi\right\rangle_{L^{2}}\right)+ \\
& +\eta\left\langle\frac{\partial}{\partial x_{4}} \varphi, \frac{\partial}{\partial x_{1}} \varphi\right\rangle_{L^{2}}+\left\|\frac{\partial}{\partial x_{1}} \varphi\right\|_{L^{2}}^{2}
\end{aligned}
$$

e, portanto,

$$
\begin{aligned}
2 \mathfrak{R e}\left\langle\frac{\partial}{\partial x_{3}} L \varphi, \frac{\partial}{\partial x_{1}} \varphi\right\rangle_{L^{2}} & =\left\langle\frac{\partial}{\partial x_{3}} L \varphi, \frac{\partial}{\partial x_{1}} \varphi\right\rangle_{L^{2}}+\overline{\left\langle\frac{\partial}{\partial x_{3}} L \varphi, \frac{\partial}{\partial x_{1}} \varphi\right\rangle_{L^{2}}} \\
& =\left\langle\frac{\partial}{\partial x_{3}} L \varphi, \frac{\partial}{\partial x_{1}} \varphi\right\rangle_{L^{2}}+\left\langle\frac{\partial}{\partial x_{1}} \varphi, \frac{\partial}{\partial x_{3}} L \varphi\right\rangle_{L^{2}} \\
& =\left\langle\frac{\partial}{\partial x_{3}} L \varphi, \frac{\partial}{\partial x_{1}} \varphi\right\rangle_{L^{2}}+\left\langle\frac{\partial}{\partial x_{3}} \varphi, \frac{\partial}{\partial x_{1}} L \varphi\right\rangle_{L^{2}} \\
& =\left\|\frac{\partial}{\partial x_{1}} \varphi\right\|_{L^{2}}^{2}+\eta\left(\left\langle\frac{\partial}{\partial x_{3}} \varphi, \frac{\partial}{\partial x_{2}} \varphi\right\rangle_{L^{2}}+\left\langle\frac{\partial}{\partial x_{4}} \varphi, \frac{\partial}{\partial x_{1}} \varphi\right\rangle_{L^{2}}\right) .
\end{aligned}
$$

Analogamente temos:

$$
\begin{aligned}
\left\langle\frac{\partial}{\partial x_{4}} L \varphi, \frac{\partial}{\partial x_{2}} \varphi\right\rangle_{L^{2}}=-\left(\left\langle\frac{\partial}{\partial x_{4}} \varphi, \frac{\partial}{\partial x_{2}} L \varphi\right\rangle_{L^{2}}\right. & \left.+\eta\left\langle\frac{\partial}{\partial x_{4}} \varphi, \frac{\partial}{\partial x_{1}} \varphi\right\rangle L^{2}\right) \\
& -\eta\left\langle\frac{\partial}{\partial x_{3}} \varphi, \frac{\partial}{\partial x_{2}} \varphi\right\rangle_{L^{2}}+\left\|\frac{\partial}{\partial x_{2}} \varphi\right\|_{L^{2}}^{2}
\end{aligned}
$$

e, portanto,

$$
2 \mathfrak{R e}\left\langle\frac{\partial}{\partial x_{4}} L \varphi, \frac{\partial}{\partial x_{2}} \varphi\right\rangle_{L^{2}}=\left\|\frac{\partial}{\partial x_{2}} \varphi\right\|_{L^{2}}^{2}-\eta\left(\left\langle\frac{\partial}{\partial x_{3}} \varphi, \frac{\partial}{\partial x_{2}} \varphi\right\rangle_{L^{2}}+\left\langle\frac{\partial}{\partial x_{4}} \varphi, \frac{\partial}{\partial x_{1}} \varphi\right\rangle_{L^{2}}\right) .
$$

\footnotetext{
${ }^{12}$ Lembrando que se $f, g \in L^{2}(\mathbb{R})$, podemos integrar por partes e obtermos $\left\langle f^{\prime}, g\right\rangle_{L^{2}}=\int f^{\prime} \bar{g}=\left.f \bar{g}\right|_{-\infty} ^{\infty}-$ $\int f \bar{g}^{\prime}=-\langle f, g\rangle_{L^{2}}$. É fácil estender o resultado para derivadas parciais em dimensões superiores.
} 
Somando os dois resultados obtemos que para todo $\varphi \in C_{c}^{\infty}\left(\mathbb{R}^{n}\right)$ vale:

$$
2 \mathfrak{R e}\left(\left\langle\frac{\partial}{\partial x_{3}} L \varphi, \frac{\partial}{\partial x_{1}} \varphi\right\rangle_{L^{2}}+\left\langle\frac{\partial}{\partial x_{4}} L \varphi, \frac{\partial}{\partial x_{2}} \varphi\right\rangle_{L^{2}}\right)=\left\|\frac{\partial}{\partial x_{1}} \varphi\right\|_{L^{2}}^{2}+\left\|\frac{\partial}{\partial x_{2}} \varphi\right\|_{L^{2}}^{2} .
$$

Note que

$$
\left\langle\frac{\partial}{\partial x_{3}} L \varphi, \frac{\partial}{\partial x_{1}} \varphi\right\rangle_{L^{2}}+\left\langle\frac{\partial}{\partial x_{4}} L \varphi, \frac{\partial}{\partial x_{2}} \varphi\right\rangle_{L^{2}}=\left\langle P_{1}(D) L \varphi, \varphi\right\rangle_{L^{2}}
$$

onde $P_{1}(D)=-\frac{\partial}{\partial x_{1}} \frac{\partial}{\partial x_{3}}-\frac{\partial}{\partial x_{2}} \frac{\partial}{\partial x_{4}}$. Já mostramos que $\|P(D) \varphi\| \geq \tilde{C}_{r}\|\varphi\|$ para $P(D)$ uma derivada direcional e $\varphi$ com suporte na bola de raio $r$ (Lembrando que a constante $\tilde{C}_{r}$ depende da direção da derivada $P(D)$ ).

Restrigindo então o domínio das funções testes, podemos repetir as contas da demonstração da Proposição 3.14 para obtermos

$$
\left\|\frac{\partial}{\partial x_{1}} \varphi\right\|_{L^{2}}^{2}+\left\|\frac{\partial}{\partial x_{2}} \varphi\right\|_{L^{2}}^{2} \geq C_{r}\|\varphi\|
$$

para $\varphi \in C_{c}^{\infty}\left(B_{r}(0)\right)$, sendo $C_{r}$ é uma constante que depende apenas do domínio $B_{r}(0)$ onde as funções não se anulam (Esta constante é a soma das constantes obtidas para as derivadas direcionais nas direções $\partial_{x_{1}}$ e $\partial_{x_{2}}$ ). Aplicando então a desigualdade de Cauchy-Schwarz à equação (3.2.17) estamos em condições de usar o Lema 3.7 e segue o resultado.

Acabamos de provar então a seguite implicação do resultado principal (Teorema 3.2):

$$
(i) \Rightarrow(i i i)
$$

onde (i) e (iii) estão repetidos abaixo.

(i) $L$ não é localmente resolúvel em nenhum ponto de $V(L)$;

(iii) O spectro de $m(L)$ é puramente imaginário e sua parte nilpotente é nula.

Para finalizar a prova do nosso resultado principal vamos demonstrar que $(i i i) \Rightarrow(i)$.

Proposição 3.17. Se todos os autovalores de $m(L)$ são puramente imaginários e a parte nilpotente de $m(L)$ se anula, então $L$ não é localmente resolúvel em nenhum ponto de $V(L)$. 
Demonstração: Como a parte real dos autovalores de $m(L)$ é sempre zero, segue que

$$
\operatorname{div}(L)=\operatorname{Tr}(m(L))=\sum_{\lambda \in \sigma(m(L))} \mathfrak{R e} \lambda=0
$$

Portanto, como já vimos, $L^{\top}=-L$. Além disso, da equação (3.2.7), podemos assumir que

$$
L=\sum_{j=1}^{p} \eta_{j} \frac{\partial}{\partial \theta_{j}}
$$

Denotemos por $\mathbb{D}=\mathbb{R}_{+} \times \mathbb{S}^{1}$ e por $\tilde{\Omega}=\mathbb{D}^{p} \times \mathbb{R}^{n-2 p}$. Temos que $L: \mathcal{D}^{\prime}(\tilde{\Omega}) \rightarrow \mathcal{D}^{\prime}(\tilde{\Omega})$. Após uma pequena mudança de coordenadas, podemos acumular as variáveis angulares nas primeiras coordenadas, de modo que $\tilde{\Omega} \cong \Omega=\mathbb{T}^{p} \times \mathbb{R}_{+}^{p} \times \mathbb{R}^{n-2 p}$, assim, podemos considerar $L$ como um operador atuando em $\mathcal{D}^{\prime}(\Omega)$ e $L^{\top}=-L$ um operador atuando em $C_{c}^{\infty}(\Omega)$. Sejam $\tau_{j}: \mathbb{S}^{1} \rightarrow \mathbb{C}, j=1, \ldots, p$ funções suaves e $\psi: \mathbb{R}_{+}^{p} \times \mathbb{R}^{n-2 p} \rightarrow \mathbb{C}$ uma função suave de suporte compacto. Defina então a seguinte função $\phi: \Omega \rightarrow \mathbb{C}$ por

$$
\phi(\theta, r, \tilde{x})=\left(\tau_{1} \otimes \cdots \otimes \tau_{p} \otimes \psi\right)(\theta, r, \tilde{x})=\tau_{1}\left(\theta_{1}\right) \ldots \tau_{p}\left(\theta_{p}\right) \psi(r, \tilde{x}) .
$$

Suponha então que $\tau_{j} \equiv 1$ para todo $j$. Neste caso, temos que $\phi \in \operatorname{Ker}\left(L^{\top}\right)$, uma vez que

$$
L^{\top} \phi=-L \phi=\sum_{j=1}^{p} \eta_{j} \frac{\partial \phi}{\partial \theta_{j}}=\sum_{j=1}^{p} \eta_{j} \frac{\partial}{\partial \theta_{j}} \psi(r, \tilde{x})=0
$$

pois $\psi$ não depende de $\theta$.

Suponha que $u \in \mathcal{D}^{\prime}(\Omega)$ e $F \in C_{c}^{\infty}(\Omega)$ são tais que $L u=F$. Isso quer dizer que para toda $\varphi \in C_{c}^{\infty}(\Omega)$ vale:

$$
\langle F, \varphi\rangle=\langle L u, \varphi\rangle=-\langle u, L \varphi\rangle .
$$

Em particular, para $\varphi=\phi$ temos que

$$
\begin{aligned}
0=-\langle u, L \phi\rangle=\langle F, \phi\rangle & =\int_{\mathbb{T}^{p} \times \mathbb{R}^{p} \times \mathbb{R}^{n-2 p}} F(\theta, r, \tilde{x}) \phi(r, \tilde{x}) d \tilde{x} d r d \theta \\
& =\int_{\mathbb{R}_{+}^{p} \times \mathbb{R}^{n-2 p}} \psi(r, \tilde{x}) \int_{\mathbb{T}^{p}} F(\theta, r, \tilde{x}) d \theta d r d \tilde{x}
\end{aligned}
$$


Segue da arbitrariedade de $\psi$ que

$$
\int_{\mathbb{T}^{p}} F(\theta, r, \tilde{x}) d \theta=0
$$

Se estivermos considerando $F$ como uma função em coordenadas cartesianas, então, após uma mudança de coordenadas temos:

$$
\int_{\mathbb{T}^{p}} F\left(r_{1} \cos \theta, \ldots, r_{p} \sin \theta_{p}, \tilde{x}\right) d \theta_{1} \ldots d \theta_{p}=0
$$

Ou seja, para que $F \in C_{c}^{\infty}$ possua solução $u \in \mathcal{D}^{\prime}, F$ precisa satisfazer condições de compatibilidade e, portanto, $L$ não é localmente resolúvel.

\subsection{Comentários sobre uma demonstração alternativa}

Quase duas décadas antes da prova do Teorema 3.2 por Treves,Müller [1992] demonstrou a equivalência das afirmações $(i)$ e $(i i i)$. Mais que isso, Müller considerou uma perturbação do operador $L$ e demonstrou o seguinte resultado:

Teorema 3.18. Seja $A=\left(a_{i j}\right)_{i, j}$ e considere o operador

$$
L_{A, b}=\sum_{i, j=1}^{n} a_{i j} x_{j} \frac{\partial}{\partial x_{i}}+b
$$

Então, $L_{A, b}$ é localmente resolúvel na origem se, e somente se, ele for $\mathcal{S}$ resolúvel, isto $e ́, \mathcal{S} \subset L_{A, b} \mathcal{S}^{\prime}$, e isto ocorre exatamente quando uma das condições a seguir é verdadeira

(i) $b \notin i \mathbb{R}$

(ii) $\sigma(A) \not \subset i \mathbb{R}$

(iii) A não é semisimples

(iv) $b \in i \mathbb{R}, \sigma(A)=\left\{i \rho_{1}, \ldots, i \rho_{s}\right\} \subset i \mathbb{R}, A$ é semisimples e os autovalores $A$, satisfazem a seguinte estimativa

$$
\left|i b-\sum_{i \rho_{j} \in \sigma(A) ; \rho_{j}>0} l_{j} \rho_{j}\right| \geq c(1+|l|)^{-m}
$$


onde $c>0$ e $m \in \mathbb{Z}_{+}$independem de $l=\left(l_{1}, \ldots, l_{r}\right) \in \mathbb{Z}_{+}^{r}$.

As técnicas utilizadas para a demonstração deste resultado foram bastante distintas das utilizadas por Treves e, nesta seção, iremos apresentar uma das idéias fundamentais da demonstração do Müller.

Nesta seção, iremos presumir uma certa familiaridade com a teoria de grupos de Lie e ágebras de Lie. Mais precisamente, vamos trabalhar com o grupo de Heisenberg e sua álgebra de Lie associada. Para uma introdução às definições mais básicas, ver apêndice C.3. Para maiores informações, consultar Humphreys [1978].

Sejam $\mathbb{H}_{n}$ o grupo de Heisenberg e $\mathfrak{h}_{\mathfrak{n}}$ a álgebra de Heisenberg. Seja ainda $\tau=i n d_{\mathbb{Z}}^{\mathbb{H}_{n}} \chi$ a representação de $\mathbb{H}_{n}$ em $L^{2}\left(\mathbb{R}^{2 n}\right)$ induzida $^{13}$ por $\chi(0,0, u)=e^{2 \pi i u}$ no centro $\mathbb{Z}$ de $\mathbb{H}_{n}$. A representação derivada também será denotada por $\tau$, e como sabemos, esta representação pode ser extendida (de maneira única) à $\mathfrak{U}\left(\mathfrak{h}_{\mathfrak{n}}\right)$. Onde $\mathfrak{U}\left(\mathfrak{h}_{\mathfrak{n}}\right)$ denota a "álgebra envelopante universal" de $\mathfrak{h}_{\mathfrak{n}}$. Iremos denotar, para $a \in \mathfrak{U}\left(\mathfrak{h}_{\mathfrak{n}}\right), \tilde{a}=\tau(a)$.

Considere ainda $\pi_{h}: \mathbb{H}_{n} \rightarrow \operatorname{Aut}\left(L^{2}\left(\mathbb{R}^{n}\right)\right)$ a representação de Schrödinger. Vamos denotar também por $\pi_{h}$ a sua representação derivada e a representação integral de Schrödinger. Neste último caso, temos que, para $F \in L^{1}\left(\mathbb{R}^{2 n}\right), \pi_{h}(F)$ é um operador integral com Kernel dado por

$$
K_{F}(x, y)=\frac{2 \pi}{h} \mathcal{F}_{1}^{-1} F\left(\frac{x+y}{4 \pi}, \frac{y-x}{h}\right)
$$

Uma ideia central no artigo do Müller é que a representação de Schrödinger preserva a propriedade de existência de solução fundamental. Mais especificamente, escolhendo $h$ conveniente e denotanto $\pi=\pi_{h}$ temos:

Teorema 3.19. Se $\tilde{a}=\tau(a)$ possui solução fundamental $F \in \mathcal{S}^{\prime}$, então $K_{F} \in \mathcal{S}\left(\mathbb{R}^{2 n}\right)$ é solução fundamental para o operador $\pi(a)$, no seguinte sentido:

Seja $T_{K_{F}}: \mathcal{S}\left(\mathbb{R}^{n}\right) \rightarrow \mathcal{S}^{\prime}\left(\mathbb{R}^{n}\right)$ a aplicação continua dada pelo kernel $K_{F}$. Então,

$$
\pi(a)\left(T_{K_{F}} \varphi\right)=\varphi \quad, \quad \forall \varphi \in \mathcal{S}\left(\mathbb{R}^{n}\right)
$$

Além disso, vale a recíproca, se $K=K_{F} \in \mathcal{S}^{\prime}\left(\mathbb{R}^{2 n}\right)$ é solução fundamental para $\pi(a)$, então F é solução fundamental para ã.

\footnotetext{
${ }^{13}$ Para saber mais sobre as representações induzidas, consultar o capítulo 6 de Folland [1994]
} 
Assim, denote por $X_{1}, \ldots X_{n}, Y_{1}, \ldots, Y_{n}, U$ a base canônica da álgebra de Heisenberg e $V_{1}, \ldots, V_{2 n}$ os campos de vetores $X_{1}, \ldots, X_{n}, Y_{1}, \ldots, Y_{n}$ nesta ordem. Sejam $J=$ $\left(\begin{array}{cc}0 & I_{n} \\ -I_{n} & 0\end{array}\right)$ e $S=\left(\begin{array}{cc}A & -B \\ C & -A^{\top}\end{array}\right) \in \mathfrak{s p}_{n}(\mathbb{R})$ uma matriz associada à uma forma simplética. Consideremos os operadores em $\mathbb{H}_{n}$ da seguinte forma

$$
\Delta_{S}=V(S J) V^{\top}=\sum_{i, j} a_{i j} V_{i} V_{j}
$$

Seja ainda $Q=\left(\begin{array}{cc}0 & \sqrt{2 \pi} I_{n} \\ \sqrt{2 \pi} I_{n} & 0\end{array}\right)$. Defina $S^{0}=Q S Q^{-1}$. Então, $S^{0}$ também pertence a $\mathfrak{s p}_{n}(\mathbb{R})$ e possui as mesmas propriedades espectrais de $S$. Seja $D$ o operador dado por,

$$
D=-i \sum_{k, l=1}^{n} b_{k l} \frac{\partial^{2}}{\partial x_{k} \partial x_{l}}+2 \sum_{k, l=1}^{n} a_{k l} x_{l} \frac{\partial}{\partial x_{k}}+i \sum_{k, l=1}^{n} c_{k l} x_{k} x_{l}+d
$$

então,

$$
2 \pi i D=\pi\left(\Delta_{S^{0}}+(d-\operatorname{tr} A) U\right)=\pi(a) \quad \operatorname{com} \quad \tilde{a}=\tilde{\Delta_{S^{0}}}+4 \pi \alpha \quad \text { e } \quad \alpha=\frac{i}{2}(d-\operatorname{tr} A)
$$

No caso particular em que $S$ é da forma $S=\left(\begin{array}{cc}A & 0 \\ 0 & -A^{\top}\end{array}\right)$, temos $4 \pi i L_{A, d}=\pi(a)$. Observe que o operador $\Delta_{S}$ é invariante pela esquerda, pois cada $V_{j}$ é invariante pela esquerda. Logo, $\Delta_{S} \in \mathfrak{U}\left(\mathfrak{h}_{\mathfrak{n}}\right)$, assim, é possível obter informações sobre a resolubilidade de $L_{A, d}$ a partir de informações sobre a resolubilidade de $\tilde{a}$. Os operadores $\Delta_{S}$ foram amplamente estudados em [Müller, Ricci, 1990] e [Müller, Ricci, 1992], fazendo uso de ferramentas como a "transformada simplética de Fourier" e a "convolução torcida", para encontrar fórmulas explícitas para o grupo a um parâmetro gerado pelo operador $(i /$ $4 \pi) \tilde{\Delta}_{S}$. Em Müller [1992], Müller faz uso destas fórmulas explícitas para construir de soluções fundamentais para o operador $\tilde{\Delta}_{S}+4 \pi \alpha$ em alguns casos, mais especificamente,

Teorema 3.20. Sejam $S \in \mathfrak{s p}_{n}(\mathbb{R}), S \neq 0$ e $\alpha \in C$. Suponha que uma das seguintes condições é satisfeita:

(i) $\alpha \notin \mathbb{R}$

(ii) $\sigma(S) \not \subset i \mathbb{R}$

(iii) $\alpha \in \mathbb{R}, \sigma(S) \subset i \mathbb{R}$, e a parte nilpotente $N$ de $S$ tem posto maior ou igual a 3 
(iv) $\alpha \in \mathbb{R}, \sigma(S) \subset i \mathbb{R}$ e $S$ é nilpotente, de modo que podemos escrever

$$
\Delta_{S}=-\sum_{j=1}^{s} \lambda_{j}\left(X_{j}^{2}+Y_{j}^{2}\right), \quad \lambda_{j} \in \mathbb{R} \backslash\{0\},
$$

com $\lambda_{j}$ satisfazendo a estimativa

$$
\left|2 \alpha+\sum_{j=1}^{s}\left(2 l_{j}+1\right) \lambda_{j}\right| \geq C(1+|l|)^{-m} \quad \forall l=\left(l_{1}, \ldots, l_{s}\right) \in \mathbb{Z}_{+}^{s}
$$

onde $C>0$ e $m \in \mathbb{Z}_{+}$não dependem de $l$.

Então, existe solução fundamental $E_{S, a} \in \mathcal{S}^{\prime}\left(\mathbb{R}^{2 n}\right)$ para o operador $\tilde{\Delta}_{S}+4 \pi \alpha$.

Este teorema implica a resolubilidade local de $L_{A, d}$ em diversos casos. Os demais casos, não contemplados por este resultado, e a recíproca são demonstrados separadamente utilizando métodos mais clássicos. 
Capítulo

4

Resolubilidade local flat de campos

vetoriais reais com singularidades

isoladas

Lema 4.1. Seja $\lambda \in \mathbb{R}$ e considere a matriz

$$
J(\lambda)=\left(\begin{array}{cccccc}
\lambda & 1 & 0 & 0 & \cdots & 0 \\
0 & \lambda & 1 & 0 & \cdots & 0 \\
\vdots & \ddots & \ddots & \ddots & \ddots & \vdots \\
0 & 0 & \cdots & 0 & \lambda & 1 \\
0 & 0 & \cdots & 0 & 0 & \lambda
\end{array}\right)=\lambda \cdot I_{n}+E
$$

Suponha que $\lambda<0$ e seja $\mu \in(0,-\lambda)$. Então existe uma constante $K \geq 0$ tal que

$$
\left\|e^{J(\lambda) t}\right\| \leq K e^{-\mu t} ; \forall t \geq 0 .
$$

Demonstração:

Observe que

$$
e^{J(\lambda) t}=e^{\left(\lambda I_{n}+E\right)}=e^{\lambda I_{n} t} e^{E t}=e^{\lambda t} I_{n} \cdot e^{E t}=e^{\lambda t} I_{n} \cdot\left(\sum_{k=0}^{\infty} \frac{(E t)^{k}}{k !}\right)
$$


${ }^{1}$ porém, $E$ é nilpotente de ordem $n$, portanto,

$$
e^{J(\lambda) t}=e^{\lambda t} \cdot\left(\sum_{k=0}^{n} \frac{(E t)^{k}}{k !}\right)=e^{\lambda t} \cdot\left(I_{n}+E t+\frac{E^{2} t^{2}}{2}+\cdots+\frac{E^{n-1} t^{n-1}}{(n-1) !}\right) .
$$

Escrevendo $a_{i}=\frac{\left\|E^{i}\right\|}{i !}, g(t)=a_{0}+a_{1} t+\cdots+a_{n-1} t^{n-1}$ e tomando $t \geq 0$ temos,

$$
\begin{aligned}
\left\|e^{J(\lambda) t}\right\| & \leq e^{\lambda t} \cdot\left(\left\|I_{n}\right\|+\|E\| t+\frac{\left\|E^{2}\right\| t^{2}}{2}+\cdots+\frac{\| E^{n-1 \|} t^{n-1}}{(n-1) !}\right) \\
& =e^{\lambda t}\left(a_{0}+a_{1} t+\cdots+a_{n-1} t^{n-1}\right)=e^{-\mu t} e^{(\mu+\lambda) t} g(t) .
\end{aligned}
$$

Como $\mu<-\lambda$ segue que $\mu+\lambda<0$ e, portanto, $\lim _{t \rightarrow \infty} e^{(\mu+\lambda) t} g(t)=0$. Assim, a função $e^{(\mu+\lambda) t} g(t)$ é limitada. Basta, então, tomar $K>0$ tal que $e^{(\mu+\lambda) t} g(t)<K$.

Existe um lema análogo e com demonstração inteiramente análoga para o caso em que a matriz em questão é um bloco de jordam associado a um autovalor complexo com parte real negativa.

Lema 4.2. Seja $\lambda=\alpha+\beta i$ e considere a matriz

$$
J(\lambda)=J(\alpha, \beta)=\left(\begin{array}{cccccc}
I_{\alpha, \beta} & I_{2} & 0 & 0 & \cdots & 0 \\
0 & I_{\alpha, \beta} & I_{2} & 0 & \cdots & 0 \\
\vdots & \ddots & \ddots & \ddots & \ddots & \vdots \\
0 & 0 & \cdots & 0 & I_{\alpha, \beta} & I_{2} \\
0 & 0 & \cdots & 0 & 0 & I_{\alpha, \beta}
\end{array}\right)
$$

onde $I_{\alpha, \beta}=\left(\begin{array}{cc}\alpha & \beta \\ -\beta & \alpha\end{array}\right)$. Suponha que $\alpha<0$ e $\beta \neq 0$ e seja $\mu \in(0,-\mathfrak{R e} \lambda)$. Então existe uma constante $K \geq 0$ tal que

$$
\left\|e^{J(\alpha, \beta) t}\right\| \leq K e^{-\mu t} ; \forall t \geq 0
$$

Lema 4.3. Seja $A$ uma matriz quadrada $n \times n$ tal que todos os autovalores de $A$ tenham ${ }^{1}$ Observe que foi possivel fazer $e^{\left(\lambda I_{n}+E\right) t}=e^{\lambda I_{n} t} e^{E t}$ pois o produto entre $I_{n} e E$ é comutativo, isto é:
$I_{n} \cdot E=E \cdot I_{n}$. 
parte real estritamente negativa. Então, existem constantes $\mu>0$ e $K \geq 0$ tais que

$$
\left\|e^{A t}\right\| \leq K e^{-\mu t}
$$

Demonstração: Seja $J_{A}$ a forma canônica de Jordan da matriz $A$ e seja $P \in G L_{n}(\mathbb{R})$ tal que $A=P^{-1} J_{A} P$. Observe que $P^{-1}\left(J_{A}\right)^{k} P=\left(P^{-1} J_{A} P\right)^{k}$ e, portanto, segue que $e^{A t}=P^{-1} e^{t J_{A}} P$. Observe que para cada $\lambda \in \sigma(A), 0<\mu_{\lambda}<-\mathfrak{R e}(\lambda)$, existe constante $K_{\lambda}>0$ tal que

$$
\left\|e^{J(\lambda) t}\right\| \leq K_{\lambda} e^{-t \mu_{\lambda}} ; \forall t \geq 0
$$

tomando $K=\max _{\lambda \in \sigma(A)}\left\{K_{\lambda}\right\}$ temos, para $\mu<\min _{\lambda \in \sigma(A)}\{\mathfrak{R e}(\lambda)\}$ e $t \geq 0$,

$$
\begin{aligned}
\left\|e^{A t}\right\| & \leq\left\|P^{-1}\right\|\left\|e^{t J_{A}}\right\|\|P\|=\left\|P^{-1}\right\|\|P\|\left\|e^{t J_{A}}\right\| \\
& =\left\|P^{-1}\right\|\|P\| \cdot \max _{\lambda \in \sigma(A)}\left\{\left\|e^{t J(\lambda)}\right\|\right\} \leq\left\|P^{-1}\right\|\|P\| K e^{-\mu t}=\tilde{K} e^{-\mu t}
\end{aligned}
$$

O seguinte lema técnico apresenta uma estimativa que será utilizada diversas vezes nos resultados a seguir

Lema 4.4. Seja $N \subseteq \mathbb{R}^{s}$ subspaço vetorial e $g: \mathbb{R}^{s} \rightarrow \mathbb{R}^{s}$ uma função flat em $N$. Seja $h: \mathbb{R}_{+} \times \mathbb{R}^{s} \rightarrow \mathbb{R}^{s}$ satisfazendo

$$
\|h(s, x)-N\| \leq C e^{-\mu s}\|x-N\| ; \forall s \geq 0, x \in D(h)
$$

para algumas constantes $\mu>0$ e $k>0$ em algum subconjunto $D(h) \subseteq \mathbb{R}^{s}$. Seja ainda $c \in \mathbb{R}$ uma consntante arbitrária. Então, para $t_{2} \geq t_{1} \geq 0, t_{0} \geq 0,\|x-N\| \leq R$ e todo $m \in \mathbb{Z}_{+}$, existem constantes $K_{m}>0$ tais que, para $x \in D(h)$ e $\|x-N\|<R$, temos

$$
\int_{t_{1}}^{t_{2}} e^{c s}\left\|g\left(h\left(s+t_{0}, x\right)\right)\right\| d s \leq \frac{C^{m} K_{m}\|x-N\|^{m} e^{-\mu m t_{0}}}{m \mu-c}\left(e^{(c-m \mu) t_{1}}-e^{(c-m \mu) t_{2}}\right)
$$

Em particular, da arbitrariedade de $m$, existe $m_{0}$ tal que $m \geq m_{0}$ implica

$$
\int_{t_{1}}^{\infty} e^{c s}\|g(h(s, x))\| d s \leq \frac{C^{m} K_{m}\|x-N\|^{m} e^{-\mu m t_{0}}}{m \mu-c} e^{(c-m \mu) t_{1}}, \quad x \in D(h),\|x-N\|<R
$$

Demonstração: Como $\mu>0, t_{2} \geq s \geq t_{1} \geq 0$ e $t_{0} \geq 0$, então, $x \in D(h)$ implica 
$\left\|h\left(s+t_{0}, x\right)-N\right\| \leq C e^{-\mu\left(s+t_{0}\right)}\|x-N\|$. Por outro lado, como $g$ é flat em $N$, então, fixada uma vizinhança limitada ${ }^{2}$ de $N$, digamos $B=\left\{x \in \mathbb{R}^{s} ;\|x-N\|<C R\right\}$, para todo $m \in \mathbb{Z}_{+}$, constantes $K_{m}$ tais que $\|g(x)\| \leq K_{m}\|x-N\|^{m}$. Assim, $\|x-N\|<R$ e $x \in D(h)$ implica $h\left(s+t_{0}, x\right) \in B$ e neste caso, para todo $m \in \mathbb{Z}_{+}$temos

$$
\left\|g\left(h\left(s+t_{0}, x\right)\right)\right\| \leq C_{m}\left(K e^{-\mu\left(s+t_{0}\right)}\|x-N\|\right)^{m}=K_{m} C^{m} e^{-\mu m\left(s+t_{0}\right)}\|x-N\|^{m}
$$

Finalmente, para $x \in D(h)$ e $\|x-N\|<R$ temos

$$
\int_{t_{1}}^{t_{2}} e^{c s}\|g(h(s, x))\| d s \leq \int_{t_{1}}^{t_{2}} e^{c s} K_{m} C^{m} e^{-\mu m\left(s+t_{0}\right)}\|x-N\|^{m} d s
$$

e segue o resultado.

Corolário 4.5. Nas condições do Lema 4.4, temos:

$$
\left\|\int_{t_{1}}^{t_{2}} e^{c s} g(h(s, x)) d s\right\| \leq \frac{C^{m} K_{m}\|x-N\|^{m}}{m \mu-c}\left(e^{(c-m \mu) t_{1}}-e^{(c-m \mu) t_{2}}\right)
$$

e, para $m$ suficientemente grande,

$$
\left\|\int_{t_{1}}^{\infty} e^{c s} g(h(s, x)) d s\right\| \leq \frac{C^{m} K_{m}\|x-N\|^{m}}{m \mu-c} e^{(c-m \mu) t_{1}}, x \in D(h),\|x-N\|<R
$$

Observação 4.6. Observe que se $g$ tem suporte compacto, então, $\|g(x)\| \leq K_{m}\|x-N\|^{m}$ para todo $x$, assim, $x \in D(h)$ implica

$$
\|g(h(s, x))\| \leq K_{m}\|h(s, x)-N\|^{m} \leq K_{m} C^{m} e^{-\mu m s}\|x-N\|^{m} .
$$

Portanto, caso $g$ tenha suporte compacto, podemos suprimir a hipótese $\|x-N\|<R$ do teorema.

Uma pequena observação, daqui por diante, vamos entender por "fluxo associado a um campo de vetore $X^{\prime \prime}$, a função $\varphi(t, x)$ que satisfaz

$$
\left(\frac{d}{d t} \varphi(t, x)\right)_{t=0}=X(x) .
$$

\footnotetext{
${ }^{2}$ Por vizinhança limitada $V$ de $N$ entendemos $\sup _{x \in V}\|x-N\|<\infty$
} 
Frequentemente, não faremos distinção do fluxo associado a um campo, e a família a um parâmetro de difeomorfismos associado ao fluxo. Em geral, denotamos a família por $U(t)$, onde $U(t) x=\varphi(t, x)$.

Definição 4.7. Sejam $A$ uma transformação linear de $\mathbb{R}^{n}$ em $\mathbb{R}^{n}$ e

$$
J(A)=\operatorname{diag}\left(J\left(\lambda_{1}\right), \ldots, J\left(\lambda_{)}\right)\right.
$$

sua forma canônica de Jordan, onde cada bloco de jordam $J\left(\lambda_{j}\right)$ é uma matriz quadrada de dimensão $m_{j}$. Sejam $E^{1}, \ldots, E^{k}$ os espaços invariantes de dimensão $m_{j}$ associados a cada bloco de Jordam ${ }^{3}$. Um subespaço invariante $W \subset \mathbb{R}^{n}$ por $A$ será chamado de espaço invariante de Jordan de $A$ se $W$ for a soma direta de espaços $E^{j}$ ou $W=\{0\}$.

Observação 4.8. Seja $A$ uma transformação linear de $\mathbb{R}^{n}$ em $\mathbb{R}^{n}$. Temos que existe uma base de $\mathbb{R}^{n}$ tal que $A$ se escreve na sua forma canônica de Jordan

$$
A=\operatorname{diag}\left(J\left(\lambda_{1}\right), \ldots, J\left(\lambda_{k}\right)\right)
$$

e os espaços invariantes $E^{j}$ associados aos blocos de Jordan são da forma

$$
\left\{\begin{array}{l}
E^{1}=\left\{x ; x_{m_{1}+1}=x_{m_{1}+2}=\cdots=x_{n}=0\right\} \\
E^{j}=\left\{x ; x_{1}=\cdots=x_{l_{j}}=x_{l_{j}+m_{j}+1}=\cdots=x_{n}=0\right\} \quad j=2, \ldots, k,
\end{array}\right.
$$

onde $m_{j}$ é a dimensão do bloco de jordan $J\left(\lambda_{j}\right)$ e $l_{j}=m_{1}+\cdots+m_{j-1}$.

Observe que nesta base, $e^{A t}=\operatorname{diag}\left(e^{J\left(\lambda_{1}\right) t}, \ldots, e^{J\left(\lambda_{)} t\right.}\right)$. Portanto, cada espaço $E^{j}$ também é um subespaço invariante de Jordan de $e^{A t}$. Em particular, obtemos que

\section{$W$ é subespaço invariante de Jordan de $A$}

$\Downarrow$

$W$ é subespaço invariante de Jordan de $e^{A t}$

Lema 4.9. Sejam $V=\sum a_{i j} x_{i} \partial_{x_{j}}$ um campo de vetores linear em $\mathbb{R}^{n}$ e seja $U(t)=e^{A t}$ a família (a um parâmetro) de difeomorfismos em $\mathbb{R}^{n}$ obtida ao integrar o campo $V$. Seja

${ }^{3}$ Os espaços $E^{j}$ são os chamados "auto espaços generalizados", e podem ser definidos por:

$$
E^{j}=\bigcup_{m=1}^{n}\left\{x \in \mathbb{R}^{n} ;\left(A-\lambda_{j} I\right)^{m} x=0\right\}
$$


$N$ um subespaço de $\mathbb{R}^{n}$ invariante de Jordan de $A=\left(a_{i j}\right)_{i, j}$ e seja $E$ o subespaço de $\mathbb{R}^{n}$ formado por todos os $x \in \mathbb{R}^{n}$ que satisfazem

$$
\|U(t) x-N\| \longrightarrow 0
$$

quando $t \rightarrow \infty$. Seja $g$ uma função suave de suporte compacto em $R^{n}$ que se anula de ordem infinita ao longo de $N$. Seja ainda $c \in \mathbb{C}$ uma constante qualquer. Defina

$$
f(x, s)=\int_{0}^{s} e^{c t} g(U(t) x) d t
$$

Então, para todos os multi indices $\alpha \in \mathbb{Z}_{+}^{n}$, e $x \in E$ o $\operatorname{limite}_{\lim _{s \rightarrow \infty}} D^{\alpha} f(x, s)$ existe e a função $f_{\alpha}: E \rightarrow \mathbb{C}$ definida por

$$
f_{\alpha}(x)=\lim _{s \rightarrow \infty} D^{\alpha} f(x, s) ; \forall x \in E
$$

é suave e se anula de ordem infinita em $N$.

\section{Demonstração:}

Observe que para fazer estimativas no integrando, a parte imaginária da constante $c$ não é relevante. Assim, ao longo desta demonstração, iremos considerar que $c$ é uma constante real.

Vamos provar inicialmente que (4.0.1) na verdade implica a seguinte desigualdade

$$
\|U(t) x-N\| \leq C e^{-t \mu}\|x-N\|
$$

Vamos supor, inicialmente, que $A$ esteja na sua forma canônica de Jordan.

$$
A=\left(\begin{array}{ccccc}
J\left(\lambda_{1}\right) & 0 & & \ldots & 0 \\
0 & J\left(\lambda_{2}\right) & & \ldots & 0 \\
\vdots & \ddots & \ddots & \ddots & \vdots \\
0 & \ldots & & J\left(\lambda_{k-1}\right) & 0 \\
0 & \ldots & & 0 & J\left(\lambda_{k}\right)
\end{array}\right)=\operatorname{diag}\left(J\left(\lambda_{1}\right), \ldots, J\left(\lambda_{k}\right)\right)
$$

onde $J\left(\lambda_{i}\right)$ representa o bloco de jordam associado ao autovalor $\lambda_{i}$. Sejam $E^{1}, \ldots, E^{k}$ os espaços invariantes associados a cada bloco de Jordan. Como $N$ é subespaço invariante de Jordan, então, $N$ é a soma direta de espaços deste tipo. Sem perda de generalidade, 
vamos supor que

$$
N=E^{l+1} \oplus \cdots \oplus E^{k}
$$

de modo que podemos escrever $\mathbb{R}^{n}=E^{1} \oplus \cdots \oplus E^{l} \oplus N$.

Como já foi observado, $N$ também é um subespaço invariante de Jordan de $e^{A t}$. Para facilitar a notação, vamos escrever

$$
U(t)=e^{A t}=\operatorname{diag}\left(e^{t J\left(\lambda_{1}\right)}, \ldots, e^{t J\left(\lambda_{k}\right)}\right)=\left(e^{t J\left(\lambda_{1}\right)}, \ldots, e^{t J\left(\lambda_{l}\right)}, e^{t J_{N}}\right)
$$

Escrevendo $x=\left(x^{1}, \ldots, x^{l}, x^{N}\right)$ e $y=\left(0, \ldots, 0, y^{N}\right) \in N$ temos que

$$
\begin{aligned}
\left\|e^{A t} x-N\right\| & =\inf _{y \in N}\left\{\left\|e^{A t} x-y\right\|\right\}=\inf _{y \in N}\left\{\left\|\operatorname{diag}\left(e^{t J\left(\lambda_{1}\right)}, \ldots, e^{t J\left(\lambda_{l}\right)}, e^{t J_{N}}\right) x-y\right\|\right\} \\
& =\inf _{y \in N}\left\{\left\|\left(e^{t J\left(\lambda_{1}\right)} x^{1}, \ldots, e^{t J\left(\lambda_{l}\right)} x^{l}, e^{t J_{N}} x^{N}\right)-\left(0, \ldots, 0, y^{N}\right)\right\|\right\} \\
& =\inf _{y \in N}\left\{\max _{j=1, \ldots, l}\left\{\left\|e^{t J\left(\lambda_{j}\right)} x^{j}\right\|,\left\|e^{t J_{N}} x^{N}-y^{N}\right\|\right\}\right\}
\end{aligned}
$$

Observe que se $x^{j} \neq 0$ temos

$$
\left\{\begin{array}{l}
\mathfrak{R e}\left(\lambda_{j}\right)>0 \quad \Rightarrow \quad\left\|e^{t J\left(\lambda_{j}\right)} x^{j}\right\| \rightarrow \infty \\
\mathfrak{R e}\left(\lambda_{j}\right)=0 \quad \Rightarrow \quad\left\|e^{t J\left(\lambda_{j}\right)} x^{j}\right\| \geq \varepsilon \text { para algum } \varepsilon>0
\end{array}\right.
$$

Assim, se $\mathfrak{R e}\left(\lambda_{j}\right) \geq 0$ e $x_{j} \neq 0$ para algum $j=1, \ldots, l$, então, $\left\|e^{A t} x-N\right\| \not \rightarrow 0$. Em particular, se $\mathfrak{R e}\left(\lambda_{j}\right) \geq 0$ para todo $j=1, \ldots, l$, então,

$$
\left\|e^{A t} x-N\right\| \rightarrow 0 \Leftrightarrow x \in N
$$

neste caso a estimativa exponencial que queríamos é trivial pois, como já vimos, $N$ é invariante por $e^{A t}$ e, portanto, $\left\|e^{A t} x-N\right\|=0$.

Vamos supor, então, que exista $\lambda_{j}$ tal que $\mathfrak{R e}\left(\lambda_{j}\right)<0$. Vamos ainda supor, sem perda de generalidade que $\mathfrak{R e}\left(\lambda_{j}\right)<0, j=1, \ldots, m$ e $\mathfrak{R e}\left(\lambda_{j}\right) \geq 0, j=m+1, \ldots, l$. Como já foi observado, se $x_{j} \neq 0$ para algum $j=m+1, \ldots, l$ então $\left\|e^{A t} x-N\right\| \nrightarrow 0$. Assim, $x \in E$ implica que $x_{m+1}=\cdots=x_{l}=0$. Neste caso, aplicando os lemas 4.1 ou 4.2 a cada bloco de Jordan, obtemos: 


$$
\begin{aligned}
\left\|e^{A t} x-N\right\| & =\inf _{y \in N}\left\{\max _{j=1, \ldots, l}\left\{\left\|e^{t J\left(\lambda_{j}\right)} x^{j}\right\|,\left\|e^{t J_{N}} x^{N}-y^{N}\right\|\right\}\right\} \leq \max _{j=1, \ldots, l}\left\{\left\|e^{t J\left(\lambda_{j}\right)} x^{j}\right\|\right\} \\
& =\max _{j=1, \ldots, m}\left\{\left\|e^{t J\left(\lambda_{j}\right)} x^{j}\right\|\right\} \leq \max _{j=1, \ldots, m}\left\{C_{j} e^{-\mu_{j} t}\left\|x^{j}\right\|\right\} \\
& \leq C e^{-\mu t} \max _{j=1, \ldots, m}\left\|x^{j}\right\|=C e^{-\mu t}\|x-N\|
\end{aligned}
$$

sendo $C=\max _{j}\left\{C_{j}\right\}$ e $\mu=\min _{j}\left\{\mu_{j}\right\}$.

No caso em que $A$ não está na forma canonica de Jordan temos $A=P^{-1} J_{A} P$ e, portanto, $e^{A t}=P^{-1} e^{t J_{A}} P$. Assim

$$
e^{A t} x-N=P^{-1} e^{t J_{A}} P x P^{-1} P-P^{-1} P N P^{-1} P=P^{-1}\left(e^{t J_{A}} w-\tilde{N}\right) P,
$$

onde $w=P x P^{-1}, \tilde{N}=P N P^{-1}$. Como $P^{-1}\left(e^{t J_{A}} w-\tilde{N}\right) P \stackrel{t \rightarrow \infty}{\longrightarrow} 0 \Leftrightarrow e^{t J_{A}} w-\tilde{N} \stackrel{t \rightarrow \infty}{\longrightarrow} 0$, reduzimos o problema ao caso anterior.

Note que $g$ se anula de ordem infinita em $N$ e tem suporte compacto. Fazendo $h(t, x)=$ $U(t) x$ e $E=D(h)$, segue do Corolário 4.5 e da Observação 4.6 que

para $s>s^{\prime} \geq 0$ temos

$$
\begin{aligned}
\left\|f(x, s)-f\left(x, s^{\prime}\right)\right\| & =\left\|\int_{s^{\prime}}^{s} e^{c t} g(U(t) x) d t\right\| \leq C \cdot K_{m}\|x-N\|^{m}\left(\frac{e^{(c-m \mu) s}-e^{(c-m \mu) s^{\prime}}}{c-m \mu}\right) \\
& =C \cdot K_{m}\|x-N\|^{m} e^{(c-m \mu) s^{\prime}}\left(\frac{e^{(c-m \mu)\left(s-s^{\prime}\right)}-1}{c-m \mu)}\right)
\end{aligned}
$$

para todo $x \in E$.

Tome $m \in \mathbb{Z}_{+}$tal que $c-m \mu<0$. Neste caso, observe que $\left(e^{(c-m \mu)\left(s-s^{\prime}\right)}-1\right)$ é limitado e temos ainda que $e^{(c-m \mu) s^{\prime}} \stackrel{s^{\prime} \rightarrow \infty}{\longrightarrow} 0$; portanto, para todo $\varepsilon>0$ existe $t_{0}>0$ tal que se $s>s^{\prime}>t_{0}$ então

$$
C \cdot K_{m}\|x-N\|^{m} e^{(c-m \mu) s^{\prime}}\left(\frac{e^{(c-m \mu)\left(s-s^{\prime}\right)}-1}{c-m \mu)}\right)<\varepsilon
$$

e, portanto, $f(x, s) \longrightarrow f_{0}(x)=\int_{0}^{\infty} e^{c t} g(U(t) x) d t$, quando $s \rightarrow \infty$. Para mostrar que a convergencia é absoluta basta obeservar que, para $m \in \mathbb{Z}_{+} \operatorname{com} c-m \mu<0$, segue 
novamente do Corolário 4.5 e da Observação 4.6 que

$$
\|f(x)-f(x, s)\|=\left\|\int_{s}^{\infty} e^{c t} g(U(t) x) d t\right\| \leq C \cdot K_{m}\|x-N\|^{m}\left(\frac{-e^{(c-m \mu) s}}{c-m \mu}\right) \stackrel{s \rightarrow \infty}{\longrightarrow} 0
$$

Finalmente, para provarmos que as derivadas de $f(x, s)$ convergem observamos que como $g$ é flat em $N$, então as derivadas de $g$ também são flat em $N$. Portanto, derivando sob o sinal de integração e aplicando a formula de Faà de Bruno ${ }^{4}$, continuamos em condições de aplicar o Corolário 4.5, de modo que podemos repetir o argumento acima para provar que

$$
\frac{\partial^{|\alpha|}}{\partial x^{\alpha}} f(x, s)=\int_{0}^{s} e^{c t} \frac{\partial^{|\alpha|}}{\partial x^{\alpha}}(g(U(t) x)) d t \underset{\text { absolutamente }}{\stackrel{s \rightarrow \infty}{\longrightarrow}} f_{\alpha}(x) ; \forall x \in E, \alpha \in \mathbb{Z}_{+}^{n} .
$$

Observe que as funções $f_{\alpha}$ são funções suaves de $x$ e temos

$$
\partial_{x}^{\beta} f_{\alpha}(x)=\lim _{s \rightarrow \infty} \partial_{x}^{\beta+\alpha} f(x, s)
$$

para toda derivada onde $\partial_{x}^{\beta} f_{\alpha}(x)$ faz sentido ${ }^{5}$. Observe que para $x \in N, f(x, s)=0$, pois

${ }^{4} \mathrm{~A}$ fórmula de Faà di Bruno é uma maneira explicita, embora um tanto quanto impraticável, de se computar a derivada de ordem arbitrária de uma composição de funções. Apesar de sua formula complexa e manuseio trabalhoso, a fórmula de Faà di Bruno é útil para se obter estimativas para as derivadas de funções compostas.

Explicitamente, sejam $u=\left(u_{1}, \ldots, u_{M}\right)$ e $z=\left(z_{1}, \ldots, z_{N}\right)$, vamos considerar uma função composta $F(u(z))$. Sejam $m=\left(m_{1}, \ldots, m_{M}\right), p=\left(p_{1}, \ldots, p_{N}\right)$ e $\tilde{p}=(|p|, \ldots,|p|)$. Sejam ainda

$$
\begin{aligned}
& A(p)=\left(\left\{0,1, \ldots, p_{1}\right\} \times \cdots \times\left\{0,1, \ldots, p_{N}\right\}\right) \backslash(\{0\} \times \cdots \times\{0\}) \\
& C(p)=\left\{m \in A(\tilde{p}) ; \sum_{l=1}^{M} m_{l} \leq|p|\right\}
\end{aligned}
$$

Além disso, seja $\phi=\left(\phi_{1}, \ldots, \phi_{M}\right), \operatorname{com} \phi_{l}: A(p) \rightarrow\left\{0,1, \ldots, m_{l}\right\}$ e

$$
V(m)=\left\{\begin{array}{ll}
\phi \mid \begin{array}{ll}
\sum_{\alpha \in A(p)} \phi_{l}(\alpha)=m_{l} & (1 \leq l \leq M) \\
\sum_{\alpha \in A(p)} \sum_{j=1}^{M} \alpha_{l} \phi_{j}(\alpha)=m_{l} & (1 \leq l \leq N)
\end{array}
\end{array}\right\}
$$

Entao, denotando $D_{z}^{p}=\partial^{\mid} p \mid / \partial z^{p}$ e $D_{u}^{m}=\partial^{|m|} / \partial u^{m}$, temos

$$
D_{z}^{p} F(u(z))=p ! \sum_{m \in C(p)}\left(D_{u}^{m} F\right)(u(z)) \sum_{\phi \in V(m)} \prod_{l=1}^{M} \prod_{\alpha \in A(p)}\left[\left(\phi_{l}(\alpha)\right) !\right]^{-1}\left(\frac{\left(D_{z}^{\alpha} u_{l}\right)(z)}{(\alpha !)}\right)^{\phi_{l}(\alpha)}
$$

Para mais informações consultar Pearce et al. [2007].

${ }^{5} \mathrm{~A}$ menos de uma mudança de coordenadas, temos $\mathbb{R}^{n}=E \oplus E^{\perp}=\mathbb{R}^{l} \times \mathbb{R}^{n-l}$, e então, podemos escrever $f_{s}=f(x, y, s)$. onde $x \in E, y \in E^{\perp}$. Assim, dizer que a derivada $\partial_{x}^{\beta} f_{\alpha}(x)$ faz sentido é dizer 
$U(t) x \in N$ e $g$ é flat em $N$. Assim, $f(x)=0$ para $x \in N$. O mesmo argumento prova ainda que o $f_{\alpha}(x)$ é uma função flat.

Definição 4.10. Considere uma variedade $X$ e um campo de vetores $V$ singular em $p \in X$. Sejam $x_{1}, \ldots, x_{n}$ coordenadas locais em torno de $p \in X$ tais que

$$
V=\sum_{i, j=1}^{n} a_{i j} x_{i} \frac{\partial}{\partial x_{j}}+o\left(|x|^{2}\right) ; x_{i}(p)=0, i=1, \ldots, n
$$

Sejam $\lambda_{1}, \ldots, \lambda_{n}$ os autovalores da matriz $\left(a_{i j}\right)^{6}$. O ponto $p$ é chamado de zero hiperbólico de $V$ se $\mathfrak{R e}\left(\lambda_{i}\right) \neq 0, i=1, \ldots, n$.

Teorema 4.11. Seja $V$ um campo vetorial linear em $\mathbb{R}^{n}$ com um zero hiperbólico na origem e seja c uma constante fixada. Então, dada uma função $g \in C^{\infty}\left(\mathbb{R}^{n}\right)$ que se anula de ordem zero na origem, existe uma função $f \in C^{\infty}\left(\mathbb{R}^{n}\right)$ que se anula de ordem infinita na origem, tal que

$$
V f+c f=g
$$

em uma vizinhança da origem.

Demonstração: Seja $U(t)$ a família a um parametro de difeomorfismos, obtida ao integrar o campo $V$, isto é, se $V=\sum_{i, j} a_{i j} x_{i} \partial_{x_{j}}$ e $A=\left(a_{i j}\right)_{i, j}$ então $U(t)=e^{t A}$. Vamos começar supondo que todos os autovalores de $A$ tem parte real negativa. Então a origem é um atrator global, isto é, para todo $x \in \mathbb{R}^{n}$ temos $U(t) x \rightarrow 0$ quando $t \rightarrow \infty$. Definina

$$
f(x)=-\int_{0}^{\infty} e^{c t} g(U(t) x) d t
$$

Considere $N=\{0\}$ e $\tilde{g}=g \chi$, onde $\chi$ é uma função suave de suporte compacto com $\chi \equiv 1$ em uma vizinhança da origem. Então, estamos em condições de aplicar o Lema 4.9 , com $E=\mathbb{R}^{n}$, de modo que a função $f$ definida acima é, não somente bem definida, como também, flat na origem.

que $\beta \in \mathbb{Z}_{+}^{l}$.

${ }^{6}$ Observe que estes autovalores independem da escolha de coordenadas 
Resta, agora, observar que $f$ é uma solução desejada. De fato,

$$
\begin{aligned}
f(U(s) x) & =-\int_{0}^{\infty} e^{c t} \tilde{g}\left(e^{A t} e^{A s} x\right) d t \\
& =-\int_{0}^{\infty} e^{c t} \tilde{g}\left(e^{A(t+s)} x\right) d t \\
& =e^{-c s}-\int_{0}^{\infty} e^{c(s+t)} \tilde{g}\left(e^{A(t+s)} x\right) d t \\
& =e^{-c s}-\int_{s}^{\infty} e^{c \tau} \tilde{g}\left(e^{A \tau} x\right) d \tau
\end{aligned}
$$

e, portanto,

$$
\begin{aligned}
\frac{d}{d s}(f(U(s) x)) & =-\left(-c e^{-c s}\right) \int_{s}^{\infty} e^{c \tau} \tilde{g}\left(e^{A \tau} x\right) d \tau-e^{-c s}\left(-e^{c \tau} \tilde{g}\left(e^{A \tau} x\right)\right)_{\tau=s} \\
& =-c f(U(s) x)+\tilde{g}(U(s) x) .
\end{aligned}
$$

Por outro lado, lembrando que $\frac{d}{d t}\left(e^{A t} x\right)=V\left(e^{A t} x\right)$ e $e^{0 A}=I_{n}$, temos

$$
\begin{aligned}
\left.\frac{d}{d s}\left(f\left(e^{A s} x\right)\right)\right|_{s=0} & =\sum_{j=1}^{n} \frac{\partial f}{\partial x_{j}}(x) \sum_{i=1}^{n} a_{i j} x_{i} \\
& =\sum_{i, j=1}^{n} a_{i j} x_{i} \frac{\partial f}{\partial x_{j}}(x)=V f(x)
\end{aligned}
$$

Fazendo $s=0$ em (4.0.9), temos

$$
V f(x)=\left.\frac{d}{d s}\left(f\left(e^{A s} x\right)\right)\right|_{s=0}=-c f\left(I_{n} x\right)+\tilde{g}\left(I_{n} x\right)=-c f(x)+\tilde{g}(x)
$$

finalizando a demonstração no caso em que $A$ tem todos os autovalores com parte real negativa ${ }^{7}$.

No caso mais geral, em que $A$ possui autovalores com parte real positiva ou negativa (mas não nula, pois a origem é um zero hiperbólico), vamos considerar $\mathbb{R}^{n}=E^{-} \oplus E^{+}$, onde $E^{-}$é soma dos espaços invariantes associados aos blocos de Jordan dos autovalores com parte real negativa e $E^{+}$a soma dos espaços invariantes associados aos blocos de Jordan dos autovalores com parte real positiva. Observe que estes espaços são espaços invariantes de Jordan. Observe ainda, que $x \in E^{-}$implica que (na verdade "se, e somente se") $e^{t A} x \rightarrow 0$ quando $t \rightarrow \infty$.

\footnotetext{
${ }^{7}$ Lembrando que $\tilde{g}=g$ em uma vizinhança da origem.
} 
Considerando, agora, a matriz $\tilde{A}=-A$, obtemos a decomposição análoga $\mathbb{R}^{n}=\tilde{E}^{-} \oplus \tilde{E}^{+}$, onde $x \in \tilde{E}^{-} \Leftrightarrow e^{-t A} x=e^{t \tilde{A}} x \rightarrow 0$ quando $t \rightarrow \infty$. Note que $\tilde{E}^{-}=E^{+} \mathrm{e}$ $\tilde{E}^{+}=E^{-}$.

Vamos supor, sem perda de generalidade, que $\tilde{E}^{-}=\left\{\left(x_{1}, \ldots, x_{n}\right) \in \mathbb{R}^{n} ; x_{l+1}=\cdots=\right.$ $\left.x_{n}=0\right\}$. Aplicando o Lema $4.9 \mathrm{com} \tilde{V}=-V=-\sum a_{i j} x_{i} \partial_{x_{j}}, N=\{0\}, E=\tilde{E}^{-}$ e $\tilde{g}=\chi g$ (sendo $\chi$ uma função de corte como anteriormente), obtemos o seguinte resultado:

Seja a função $f: \tilde{E}^{-} \oplus \tilde{E}^{+} \equiv \mathbb{R}^{l} \times \mathbb{R}^{n-l} \rightarrow \mathbb{C}$ definida por

$$
f(x, y, s)=\int_{0}^{s} e^{c t} \tilde{g}\left(e^{t \tilde{A}}(x, y)\right) d t
$$

Então,

$$
\frac{\partial^{|\alpha|}}{\partial x^{\alpha}} f(x, y, s) \underset{\text { absolutamente }}{\stackrel{t \rightarrow \infty}{\longrightarrow}} f_{\alpha}(x) ; \forall(x, y) \in \tilde{E}^{-} \times\{0\}, \alpha \in \mathbb{Z}_{+}^{n},
$$

onde cada $f_{\alpha}(x)$ é uma função suave, definida em $\tilde{E}^{-} \equiv \mathbb{R}^{l}$, que se anula de ordem infinita na origem. Em particular, isso é verdade para $\alpha=0=(0, \ldots, 0)$ e temos que $f_{0}(x)$ satisfaz $\partial^{\beta} f_{0}(x)=f_{(\beta, 0)}$ para todo $\beta \in \mathbb{Z}_{+}^{l}$.

Considere, agora, $(x, y) \in \mathbb{R}^{n}=\tilde{E}^{-} \oplus \tilde{E}^{+}$. Queremos estender o mapa $f_{0}$ a uma função $\tilde{f}$ definida em todo $\mathbb{R}^{n}$, tal que $\partial^{\alpha} \tilde{f}(x, 0)=f_{\alpha}(x)$ para todo $\alpha \in \mathbb{Z}_{+}^{n}$. Observe que basta definirmos em uma vizinhança de $\tilde{E}^{-} \equiv \mathbb{R}^{l}$. Depois estendemos com o auxílio de uma função com suporte nesta vizinhança e com valor um em $\tilde{E}^{-}$. Para isso, considere uma função $\rho \in \mathbb{C}_{c}^{\infty}(\mathbb{R})$ com suporte no intervalo $(-1,1), 0 \leq \rho \leq 1$ e tal que $\rho \equiv 1$ em uma vizinhança da origem. Defina

$$
\begin{aligned}
\tilde{f}: \tilde{E}^{-} \oplus \tilde{E}^{+} & \longrightarrow \mathbb{C} \\
(x, y) & \mapsto \tilde{f}(x, y)=\sum_{\gamma \in \mathbb{Z}_{+}^{n-l}} \frac{y^{\gamma}}{\gamma !} f_{(0, \gamma)}(x) \rho\left(\left\|f_{(0, \gamma)}(x)\right\|^{2}\|y\|^{2}\right) \\
& =\sum_{\left(0, \ldots, 0, \alpha_{l+1}, \ldots, \alpha_{n}\right) \in \mathbb{Z}_{+}^{n}} \frac{(x, y)^{\alpha}}{\alpha !} f_{\alpha}(x) \rho\left(\left\|f_{\alpha}(x)\right\|^{2}\|y\|^{2}\right)
\end{aligned}
$$

Precisamos mostrar que $\tilde{f}$ está bem definida em uma vizinhança de $\tilde{E}^{-}$. Para isso, 
vamos mostrar que a série converge absolutamente para $\|y\|<1$. Observe que, se

$$
\left\|f_{(0, \gamma)}(x)\right\|^{2}\|y\|^{2}>\left\|f_{(0, \gamma)}(x)\right\|\|y\|>1
$$

então o $(0, \gamma)$-ésimo termo é zero e, assim, podemos supor que $\left\|f_{(0, \gamma)}(x)\right\|\|y\| \leq 1$. Além disso, $0 \leq \rho\left(\left\|f_{(0, \gamma)}(x)\right\|^{2}\|y\|^{2}\right) \leq 1$. Com isso, para $|\gamma| \geq 1$ temos

$$
\frac{\|y\|^{|\gamma|}}{\gamma !}\left\|f_{(0, \gamma)}(x)\right\| \rho\left(\left\|f_{(0, \gamma)}(x)\right\|^{2}\|y\|^{2}\right) \leq \frac{\|y\|^{|\gamma|-1}}{\gamma !}\left\|f_{(0, \gamma)}(x)\right\| y \| \leq \frac{\|y\|^{|\gamma|-1}}{\gamma !}
$$

e, portanto,

$$
\begin{aligned}
\|\tilde{f}(x, y)\| & \leq \sum_{\gamma \in \mathbb{Z}_{+}^{n-l}} \frac{\|y\|^{|\gamma|}}{\gamma !}\left\|f_{(0, \gamma)}(x)\right\| \rho\left(\left\|f_{(0, \gamma)}(x)\right\|^{2}\|y\|^{2}\right) \leq \sum_{\gamma \in \mathbb{Z}_{+}^{n-l}} \frac{\|y\|^{|\gamma|}}{\gamma !}\left\|f_{(0, \gamma)}(x)\right\| \\
& =\left\|f_{(0)}(x)\right\|+\sum_{|\gamma| \geq 1 ; \gamma \in \mathbb{Z}_{+}^{n-l}} \frac{\|y\|^{|\gamma|}}{\gamma !}\left\|f_{(0, \gamma)}(x)\right\| \\
& \leq\left\|f_{(0)}(x)\right\|+\sum_{|\gamma| \geq 1 ; \gamma \in \mathbb{Z}_{+}^{n-l}} \frac{\|y\|^{|\gamma|-1}}{\gamma !} \\
& \leq\left\|f_{(0)}(x)\right\|+\sum_{|\gamma| \geq 1 ; \gamma \in \mathbb{Z}_{+}^{n-l}} \frac{1}{\gamma !} .
\end{aligned}
$$

Como a última soma é numérica e convergente, segue do $M$ teste de Weierstrass que $\tilde{f}$ está bem definida e a convergência é absoluta.

O mesmo processo pode ser utilizado para mostrar que, derivando a série, termo a termo, continuamos com uma série absolutamente convergente para $\|y\|<1$. 
Vamos calcular $\frac{\partial}{\partial x_{i}} \tilde{f}(x, 0)$ :

$$
\begin{aligned}
\frac{\partial}{\partial x_{i}} \tilde{f}(x, y)= & \sum_{\gamma \in \mathbb{Z}_{+}^{n-l}} \frac{\partial}{\partial x_{i}}\left(\frac{y^{\gamma}}{\gamma !} f_{(0, \gamma)}(x) \rho\left(\left\|f_{(0, \gamma)}(x)\right\|^{2}\|y\|^{2}\right)\right) \\
=\sum_{\gamma \in \mathbb{Z}_{+}^{n-l}} \frac{y^{\gamma}}{\gamma !}\left(\frac{\partial f_{(0, \gamma)}}{\partial x_{i}}(x) \rho\left(\left\|f_{(0, \gamma)}(x)\right\|^{2}\|y\|^{2}\right)+\right. & \\
& \left.\quad+f_{(0, \gamma)}(x) \rho^{\prime}\left(\left\|f_{(0, \gamma)}(x)\right\|^{2}\|y\|^{2}\right)\|y\|^{2} \frac{\partial}{\partial x_{i}}\left(\left\|f_{(0, \gamma)}(x)\right\|^{2}\right)\right)
\end{aligned}
$$

portanto,

$$
\frac{\partial}{\partial x_{i}} \tilde{f}(x, 0)=\frac{\partial f_{(0,0)}}{\partial x_{i}}(x)=f_{e_{i}}(x)
$$

onde $e_{i}$ é o $i$-ésimo vetor da base canônicade $\mathbb{R}^{n}$.

Além disso,

$$
\begin{aligned}
\frac{\partial}{\partial y_{j}} \tilde{f}(x, 0) & =\left.\sum_{\gamma \in \mathbb{Z}_{+}^{n-l}} \frac{\partial}{\partial y_{j}}\right|_{y=0}\left(\frac{y^{\gamma}}{\gamma !} f_{(0, \gamma)}(x) \rho\left(\left\|f_{(0, \gamma)}(x)\right\|^{2}\|y\|^{2}\right)\right) \\
& =\left.\sum_{\gamma \in \mathbb{Z}_{+}^{n-l}} \frac{\partial}{\partial y_{j}}\right|_{y=0}\left(\frac{y^{\gamma}}{\gamma !} f_{(0, \gamma)}(x)\right)=f_{e_{l+j}}(x)
\end{aligned}
$$

Analogamente, se obtem que $\partial^{\alpha} \tilde{f}(x, 0)=f_{\alpha}(x)$. Considere agora a função $h=V \tilde{f}+c \tilde{f}-\tilde{g}$. Repetindo as contas feita em (4.0.5), (4.0.9), (4.0.11) e (4.0.13), obtemos que $h$ é flat em $\tilde{E}^{-}$.

Lembrando que $\tilde{E}^{-}=E^{+}$e $\tilde{E}^{+}=E^{-}$são invariantes por $e^{t A}$ e $e^{-t A}$, temos que

$$
\begin{aligned}
\left\|e^{t A}(x, y)-\tilde{E}^{-}\right\| & =\|e^{t A}(x, 0)+\underbrace{e^{t A}(0, y)-\tilde{E}^{-}}_{\in \tilde{E}^{-}}\| \\
& =\left\|e^{t A}(x, 0)-\tilde{E}^{-}\right\| ; \forall(x, y) \in E^{-} \oplus E^{+} \equiv \mathbb{R}^{n} ;
\end{aligned}
$$

$\operatorname{Mas}(x, y) \in E^{-} \subseteq \mathbb{R}^{n} \Leftrightarrow e^{t A}(x, y) \rightarrow 0$ quando $t \rightarrow \infty$, de modo que

$$
\left\|e^{t A}(x, y)-E^{+}\right\|=\left\|e^{t A}(x, 0)-E^{+}\right\| \rightarrow 0 ; \forall(x, y) \in E^{-} \oplus E^{+} \equiv \mathbb{R}^{n}
$$


Podemos aplicar novamente o Lema 4.9, utilizando $E=\mathbb{R}^{n}, N=E^{+}, h$ a função flat ao longo de $N$ e $V$ o campo original (com $\left.U(t)=e^{t A}\right)$ dado. Obtemos com isso uma função $\tilde{h} \in C^{\infty}\left(\mathbb{R}^{n}\right)$ definida pela integral imprópria

$$
\tilde{h}(x, y)=-\int_{0}^{\infty} e^{c t} h\left(e^{t A}(x, y)\right) d t
$$

Repetindo a conta anterior, vemos que $\tilde{h}$ satisfaz $V \tilde{h}+c \tilde{h}=h$ e, portanto, satisfaz

$$
0 \equiv V \tilde{h}+c \tilde{h}-h=V \tilde{h}+c \tilde{h}-(V \tilde{f}+c \tilde{f}-\tilde{g})=V(\tilde{h}-\tilde{f})+c(\tilde{h}-\tilde{f})-\tilde{g}
$$

Assim, em uma vizinhança da origem, $g=V(\tilde{h}-\tilde{f})+c(\tilde{h}-\tilde{f})$, onde $\tilde{h}$ e $\tilde{f}$ são funções suaves em $\mathbb{R}^{n}$, provando o teorema. 


\section{Capítulo}

\section{Teorema de Linearização de Sternberg}

Queremos, agora, estudar o lema (4) do artigo [Silva, dos Santos Filho, 2006]. Em resumo, este lema nos fornece condições necessárias para que o operador $L+c: C^{\infty}\left(\mathbb{R}^{n}\right) \rightarrow C^{\infty}\left(\mathbb{R}^{n}\right)$, sendo $L$ um campo vetorial com singularidade isolada e coeficientes em $C^{\infty}\left(\mathbb{R}^{n} ; \mathbb{R}\right)$ e $c \in C^{\infty}\left(\mathbb{R}^{n}\right)$. No entanto, para que se compreenda a necessidade de cada hipótese do lema, é necessário estudar primeiramente o Teorema de Linearização de Sternberg. Vamos dedicar, então, esta seção ao estudo deste poderoso resultado. A demonstração que estudaremos foi apresentada por E. Nelson [Nelson, 1969]. Uma observação pertinente é que o trabalho de Nelson precede Guillemin, Schaeffer [1977] e, de fato, foi inspiração para a demonstração do Teorema 4.11. Os mecanismos de algumas das demonstrações a seguir serão muito semelhantes aos já vistos anteriormente mas, como acabamos de observar, foram na verdade as fontes de inspiração da demonstração que fizemos no capítulo 4 .

Vamos fixar algumas notações:

Seja $L_{\text {sym }}^{j}\left(\mathbb{R}^{s}\right)$ o conjunto das transformações $j$-lineares e simétricas em $\mathbb{R}^{s}$ e para $k=1,2, \ldots$ defina

$$
\tilde{\mathcal{A}}^{k}:=\left\{\begin{array}{l|l}
R: \mathbb{R}^{s} \rightarrow \mathbb{R}^{s} & \begin{array}{l}
R(x)=\sum_{j=1}^{k} A_{j} x^{j} ; A_{j} \in L_{\text {sym }}^{j}\left(\mathbb{R}^{s}\right) ; \\
R(0)=0 ; D R(0) \text { inversível }
\end{array}
\end{array}\right\}
$$


Observe que $\tilde{\mathcal{A}}^{k}$ com a operação "composição + truncamento"1 é um grupo. Podemos definir, para $k=\infty, \tilde{\mathcal{A}}^{\infty}$ como o conjunto de todas as séries formais da forma

$$
\sum_{j=1}^{\infty} A_{j} x^{j} ; A_{j} \in L_{\text {sym }}^{j}\left(\mathbb{R}^{s}\right),
$$

com $A_{1}$ inversível. $\tilde{\mathcal{A}}^{\infty}$ também é um grupo com a operação dada pela composição formal. Note ainda que, se $1 \leq k \leq k^{\prime} \leq \infty$, então $\tilde{\mathcal{A}}^{k^{\prime}} \hookrightarrow \tilde{\mathcal{A}}^{k}$, onde $\hookrightarrow$ indica o truncamento da série, é um homomorphismo sobrejetor.

Defina, para cada $k=1, \ldots, \infty$, o conjunto $\mathcal{S}^{k}$ de todos os germes $R$ de funções $C^{k}$ na origem em $\mathbb{R}^{s}$ que fixem a origem e tal que $D R(0)$ seja inversível. Segue do teorema da função inversa que $\mathcal{S}^{k}$ é um grupo com a operação dada pela composição. Observe ainda que, se $1 \leq k \leq k^{\prime} \leq \infty$, então $\mathcal{S}^{k^{\prime}} \hookrightarrow \mathcal{S}^{k}$, onde $\hookrightarrow$ indica a inclusão, é um homomorphismo sobrejetor.

Finalmente, observe que, para cada $k \in \mathbb{Z}_{+}, \mathcal{S}^{k} \hookrightarrow \tilde{\mathcal{A}}^{k}$, onde $\hookrightarrow$ significa "tomar a série de Taylor de ordem $k^{\prime \prime}$, é um homomorphismo sobrejetor. Isso é verdade também para $k=\infty$, e será provado no lema a seguir.

Lema 5.1. Seja $A_{j} \in L_{\text {sym }}^{j}\left(\mathbb{R}^{s}\right)$ para $j=1,2, \ldots$ Então, existe uma aplicação suave $F: \mathbb{R}^{s} \rightarrow \mathbb{R}^{s}$, cuja série de Taylor na origem é dada por $\sum_{j} A_{j} x^{j}$.

Demonstração: Observe que só precisamos definir a função $F$ em uma vizinhança $U$ da origem, pois podemos depois multiplicar $F$ por uma função de suporte compacto, igual a 1 em uma vizinhança $V \subset U$ da origem, e com suporte em $U$. Estendendo a função $F$ a zero em todo $\mathbb{R}^{s} \backslash U$. Consideremos agora uma função suave $\rho: \mathbb{R} \rightarrow \mathbb{R} \operatorname{com}, 0 \leq \rho \leq 1$, $\rho \equiv 1$ em uma vizinhança da origem $\operatorname{esupp}(\rho) \subset[-1,1]$. Definimos então

$$
F(x)=\sum_{j=1}^{\infty} A_{j} x^{j} \rho\left(\left\|A_{j}\right\|\|x\|^{2}\right) .
$$

Note que se $x=0$, então todos os termos da série se anulam e $F(0)=0$. Vamos supor então que $x \neq 0$. Observe que para $\left\|A_{j}\right\|\|x\|^{2}>1$, o $j$-ésimo termo da série é nulo. De

\footnotetext{
${ }^{1}$ Por truncamento, entenda que estamos desconsiderando os termos de ordem $>k$
} 
forma que podemos assumir que $\left\|A_{j}\right\| \leq\|x\|^{-2}$ e, assim,

$$
\begin{aligned}
\sum_{j=0}^{\infty}\left\|A_{j}\right\|\|x\|^{j} \rho\left(\left\|A_{j}\right\|\|x\|^{2}\right) & =\left\|A_{0}\right\|+\left(\left\|A_{1}\right\|\|x\|\right)+\sum_{j=2}^{\infty}\left\|A_{j}\right\|\|x\|^{j} \rho\left(\left\|A_{j}\right\|\|x\|^{2}\right) \\
& \leq\left\|A_{0}\right\|+\left(\left\|A_{1}\right\|\|x\|\right)+\sum_{j=2}^{\infty}\|x\|^{j-2} .
\end{aligned}
$$

Portanto, a série $F(x)$ é absolutamente convergente para $\|x\|<1$. Derivando termo a termo, obtemos que a série dos termos derivados também é absolutamente convergente para $\|x\|<1$, portanto $F$ é suave. Finalmente, observe que como $\alpha$ é 1 em uma vizinhança da origem, entao, a série de Taylor de $F$ é dada por $\sum A_{j} x^{j}$ como queríamos demonstrar.

Mostramos assim que o seguinte diagrama é comutativo:

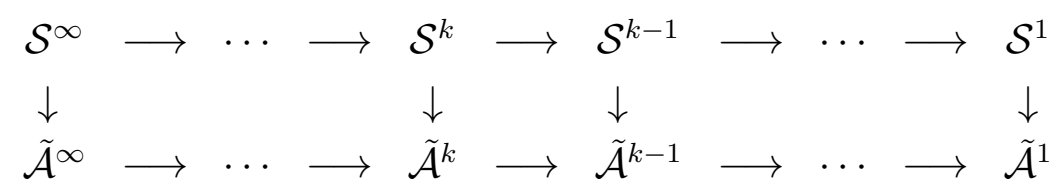

Estamos interessados em linearizar, atravez de conjugações, elementos em $\mathcal{S}^{k}$, isto é, dado $T \in \mathcal{S}^{k}$, encontrar $R \in \mathcal{S}^{k}$ tal que $R T R^{-1}$ seja linear. O próximo resultado estabelece condições suficientes para a linearização em $\tilde{\mathcal{A}}^{k}$.

Teorema 5.2. Seja $T \in \tilde{\mathcal{A}}^{k}$ para algun $k=1,2, \ldots, \infty$ com parte linear $T_{1}$. Sejam $\mu_{1}, \ldots, \mu_{s}$ autovalores complexos de $T_{1}$ (incluindo suas multiplicidades) e denote $\mu=$ $\left(\mu_{1}, \ldots, \mu_{s}\right) \in \mathbb{C}^{s}$. Seja ainda o conjunto $\Gamma^{j}=\left\{\alpha \in \mathbb{Z}_{+}^{s} ;|\alpha|=j\right\}$. Suponha que $\forall i=$ $1, \ldots, s$ tenhamos

$$
\mu_{i} \neq \mu^{\alpha} ; \forall \alpha \in \Gamma^{j}, j=2, \ldots, k
$$

Então, existe uma única aplicação $R \in \tilde{\mathcal{A}}^{k}$, com parte linear $R_{1}=I d$, tal que $R T R^{-1}=T_{1}$.

Demonstração: Observe que se $R_{1}=I d$, então $R$ é automaticamente inversível e, portanto, $R T R^{-1}=T_{1} \Leftrightarrow R T=T_{1} R$.

Primeiramente, vamos considerar $\mathbb{R}^{s}$ um espaço vetorial sobre o corpo dos complexos, 
e vamos supor que $T_{1}$ é diagonalizável sobre $\mathbb{C}$, com autovalores complexos $\mu_{1}, \ldots, \mu_{s}$, e consideremos um sistema de coordenadas onde $T_{1}$ é diagonalizável. Neste caso, temos $\left(T_{1} x\right)_{i}=\mu_{i} x_{i}$.

Como já vimos, precisamos resolver uma equação do tipo $R T=T_{1} R$, sendo $R x=\sum_{j} R_{j} x^{j}, T x=\sum_{j} T_{j} x^{j}$ e $R_{1}=I d$. Queremos, então, determinar, para cada $j=1,2, \ldots, k$, as aplicações $R_{j}$ que satisfazem $T_{1} R x=R T x$ para todo $x \in \mathbb{R}^{s}$. Isso será feito indutivamente, isto é, uma vez determinados $R_{1}, \ldots, R_{j-1}$ queremos definir $R_{j}$. Explicitamente, queremos resolver:

$$
\begin{aligned}
& T_{1}\left(x+R_{2} x^{2}+R_{3} x^{3}+\ldots\right)= \\
& \left(T_{1} x+T_{2} x^{2}+\ldots\right)+R_{2}\left(T_{1} x+T_{2} x^{2}+\ldots\right)^{2}+R_{3}\left(T_{1} x+T_{2} x^{2}+\ldots\right)^{3}+\ldots
\end{aligned}
$$

Comparando os coeficientes $x^{j}$, podemos escrever

$$
T_{1}\left(R_{j} x^{j}\right)=R_{j}\left(T_{1} x\right)^{j}+H_{j} x
$$

onde $H_{j}: \mathbb{R}^{s} \rightarrow \mathbb{R}^{s}$ é uma aplicação que depende de $R_{1}, \ldots, R_{j-1}$ e, portanto, pela hipótese de indução já está unicamente determinado. Observe que $H_{j} x=\left(h_{j}^{1}(x), \ldots, h_{j}^{s}(x)\right)$ é um polinômio homogêneo de ordem $j$, e podemos escrever

$$
h_{j}^{i}(x)=\sum_{\alpha \in \Gamma^{j}} f_{i, j, \alpha} x^{\alpha}, i=1,2, \ldots, s
$$

onde $f_{i, j, \alpha} \in \mathbb{R}$ são as constantes que definem $H_{j}$.

Suponha agora que $R_{j} x^{j}$ se escreve explicitamente da seguinte forma:

$$
\left(R_{j} x^{j}\right)_{i}=\sum_{\alpha \in \Gamma^{j}} r_{\alpha}^{i} x^{\alpha}, i=1, \ldots, s
$$

onde $r_{\alpha}^{i} \in \mathbb{R}$ são os coeficientes que determinam a aplicação $R_{j}$. Então, a equação (5.0.1) pode ser escrita como

$$
\begin{aligned}
\mu_{i} \sum_{\alpha \in \Gamma^{j}} r_{\alpha}^{i} x^{\alpha} & =\left(T_{1}\left(R_{j} x^{j}\right)\right)_{i}=\left(R_{j}\left(T_{1} x\right)^{j}\right)+\left(H_{j-1} x\right)_{i} \\
& =\sum_{\alpha \in \Gamma^{j}} r_{\alpha}^{i}(T x)^{\alpha}+h_{j}^{i}(x)=\sum_{\alpha \in \Gamma^{j}} r_{\alpha}^{i} \mu^{\alpha} x^{\alpha}+\sum_{\alpha \in \Gamma^{j}} f_{i, j, \alpha} x^{\alpha} .
\end{aligned}
$$


Comparando termo a termo, obtemos a equação $\mu_{i} r_{\alpha}^{i}=\mu^{\alpha} r_{\alpha}^{i}+f_{i, j, \alpha}$. Então,

$$
\left(\mu_{i}-\mu^{\alpha}\right) r_{\alpha}^{i}=f_{i, j, \alpha}, \forall j=2,3, \ldots, i=1, \ldots, s, \alpha \in \Gamma^{j} .
$$

Como $\mu_{i} \neq \mu^{\alpha}$, para quaiquer $i=1, \ldots, s$ e $\alpha \in \Gamma^{j}$, segue que a equação acima possui uma única solução $r_{\alpha}^{i}$. Provamos, assim, a existência e a unicidade de $R$ no caso em que $T_{1}$ é diagonalizável sobre $\mathbb{C}$. Além disso, segue da unicidade de $R$ que $R$ é real, pois $R T R^{-1}=T_{1} \Leftrightarrow \overline{R T R^{-1}}=\bar{T}_{1}$ e como $T$ e $T_{1}$ são reais, temos $\bar{R} T \overline{R^{-1}}=T_{1}$. Logo, $R=\bar{R}$.

Precisamos, agora, mostrar que o resultado continua válido quando $T_{1}$ não é diagonalizável. Denote por $\mathcal{L}\left(L_{\text {sym }}^{j}\left(\mathbb{R}^{s}\right)\right)$ o conjunto das transformações lineares $F$ : $L_{\text {sym }}^{j}\left(\mathbb{R}^{s}\right) \rightarrow L_{\text {sym }}^{j}\left(\mathbb{R}^{s}\right)$. Considere a seguinte aplicação

$$
\begin{aligned}
& \Phi: G L_{s}(\mathbb{R}) \rightarrow \mathcal{L}\left(L_{\text {sym }}^{j}\left(\mathbb{R}^{s}\right)\right) \\
& A \mapsto \Phi(A): L_{\text {sym }}^{j}\left(\mathbb{R}^{s}\right) \rightarrow L_{\text {sym }}^{j}\left(\mathbb{R}^{s}\right) \\
& R_{j} \mapsto\left(\Phi(A) R_{j}\right) x^{j}=A\left(R_{j} x^{j}\right)-R_{j}(A x)^{j} .
\end{aligned}
$$

Note que podemos reescrever (5.0.1) da seguinte forma

$$
\left(\Phi\left(T_{1}\right) R_{j}\right) x^{j}=H_{j}(x)
$$

Suponha $A \in G L_{s}(\mathbb{R})$ diagonal, com autovalores $\mu_{1}, \ldots, \mu_{s}$, e seja $\lambda$ autovalor de $\Phi(A)$. Então, existe $R_{j} \in L_{\text {sym }}^{j}\left(\mathbb{R}^{s}\right)$ não nulo, $\operatorname{com}\left(R_{j} x^{j}\right)_{i}=\sum_{\alpha \in \Gamma^{j}} r_{\alpha}^{i} x^{\alpha}$, tal que

$$
\left((\lambda-\Phi(A)) R_{j}\right) x^{j}=0 \quad, \forall x \in \mathbb{R}^{s}
$$

$\log 0$,

$$
\lambda \cdot \sum_{\alpha \in \Gamma^{j}} r_{\alpha}^{i} x^{\alpha}=\left(\lambda \cdot\left(R_{j} x^{j}\right)\right)_{i}=\left(\left(\Phi(A) R_{j}\right) x^{j}\right)_{i}=\mu_{i} \sum_{\alpha \in \Gamma^{j}} r_{\alpha}^{i} x^{\alpha}-\sum_{\alpha \in \Gamma^{j}} r_{\alpha}^{i} \mu^{\alpha} x^{\alpha}
$$

Portanto, os autovalores de $\Phi(A)$ são da forma $\lambda=\mu_{i}-\mu^{\alpha}$, com $i=1, \ldots, s$ e $\alpha \in \Gamma^{j}$. Seja $A \in G L_{s}(\mathbb{R})$, com autovalores $\mu_{1}, \ldots, \mu_{s}$. Vamos denotar por $\Lambda^{j}(A)=\{\lambda \in \mathbb{C} ; \lambda=$ $\left.\mu_{i}-\mu^{\alpha} ; i=1, \ldots, s ; \alpha \in \Gamma^{j}\right\}$. Denotemos ainda por $\mathcal{P}_{k}$ o espaço dos polinômios de grau $k$ e seja $d$ a dimensão do espaço vetorial real $L_{\text {sym }}^{j}\left(\mathbb{R}^{s}\right)$. Finalmente, se $L: V \rightarrow V$ é uma transformação linear, denotemos por $p_{L}(x)$ seu polinômio característico. Considere, 
agora, a seguinte aplicação contínua ${ }^{2}$

$$
\begin{aligned}
g: G L_{s}(\mathbb{R}) & \rightarrow \mathcal{P}_{d} \\
A & \mapsto g(A)=p_{\Phi(A)}(x)-\prod_{\lambda \in \Lambda^{j}(A)}(x-\lambda) .
\end{aligned}
$$

Observe que se $A$ é diagonalizável, então $\sigma(\Phi(A)$ ) (o espectro de $\Phi(A)$ ) é o conjunto $\Lambda^{j}(A)$, e neste caso temos que $g(A)=0^{3}$. Lembrando que o conjunto das matrizes $s \times s$ diagonalizáveis é denso em $G L_{s}(\mathbb{R})$, segue da continuidade de $g$ que $g(A)$ é constante para toda $A \in G L_{s}(\mathbb{R})$. Assim, $\sigma(\Phi(A))=\Lambda^{j}(A)$ para todo $A \in G L_{s}(\mathbb{R})$. Por hipótese, temos que $0 \notin \Lambda^{j}(A)$ e, portanto, $\Phi(A)$ é sempre inversível. Assim, a equação (5.0.2) possui única solução $R_{j}$.

Consideremos, agora, $B$ uma vizinhança aberta da origem e um campo de vetores $X: B \rightarrow \mathbb{R}^{s}$. Seja $\varphi(t, x)=U(t) x$ o fluxo local deste campo de vetores. Se $R$ é um difeomorfismo local que fixa a origem então podemos definir o campo $Y=D_{*} X$ por

$$
Y(y)=D R\left(R^{-1} y\right) \cdot X\left(R^{-1} y\right)
$$

com fluxo local dado por $\left(R_{*} \varphi\right)(t, y)=R\left(\varphi\left(t, R^{-1} y\right)\right)=R U(t) R^{-1} y$.

Lema 5.3. Sejam $X$ campo de vetores $C^{k}$ e $\varphi_{t}(x)=\varphi(t, x)$ seu fluxo. Suponha que, $\varphi(t, 0)=0$ para $t$ suficientemente pequeno, digamos $|t|<\varepsilon$, suponha ainda que

$$
D^{j} \varphi_{t}(0) x^{j}=0 \quad j=1, \ldots, k \quad|t|<\varepsilon
$$

Então, $X(0)=0$ e $D^{j} X(0)=0$ para $j=1, \ldots, k$.

Demonstração: Observe que, $X(0)=0$ trivialmente, pois $\left.\frac{d \varphi}{d t}(0, x)\right|_{x=0}=0$ uma vez que $\varphi(t, 0)=0$ para $|t|<\varepsilon$. Assim, o lema é verdadeiro para $k=0$. Suponha por indução, que o lema seja verdadeiro para $k-1$. Daí, definindo $\psi(t, x)=D \varphi_{t}(x)$, consideramos o

\footnotetext{
${ }^{2}$ Para provar a continuidade de $g$, observe que a aplicação $\Phi$ é contínua e a aplicação que associa uma matriz a seu polinômio característico é contínua, de modo que o termo $p_{\Phi(A)}(x)$ é uma função contínua de $A$. Além disso, as raízes de um polinômio variam continuamente em função dos coeficientes do polinômio, de modo que os autovalores de uma matriz variam continuamente em função dos termos da matriz. Assim, o produtório $\prod_{\lambda \in \Lambda^{j}(A)}(x-\lambda)$ também é uma função contínua de $A$, pois ele não faz distinção entre os autovalores de $A$.

${ }^{3}$ Note que $g(A)$ é na verdade a diferença de dois polinômios mônicos do mesmo grau, e no caso em que $A$ é diagonalizável, então estes polinômios possuem exatamente as mesmas raizes, logo são iguais.
} 
campo determinado por

$X^{\prime}\left(\begin{array}{c}x \\ \xi\end{array}\right)=\left.\frac{d}{d t}\right|_{t=0}\left(\begin{array}{c}\varphi(t, x) \\ \psi(t, x) \cdot \xi\end{array}\right)=\left(\begin{array}{c}X(\varphi(t, x)) \\ D X(\varphi(t, x)) \cdot \psi(t, x) \cdot \xi\end{array}\right)_{t=0}=\left(\begin{array}{c}X(x) \\ D X(x) \cdot \xi\end{array}\right)$.

Temos que $X^{\prime}$ é um campo $C^{k-1}$ e com fluxo $\Phi_{t}(x, \xi)$ dado por $\Phi_{t}(x, \xi)=$ $\left(\begin{array}{c}\varphi(t, x) \\ \psi(t, x) \cdot \xi\end{array}\right)$. Por hipótese, temos que

$$
D^{j} \Phi_{t}(0,0)\left(\begin{array}{c}
x \\
\xi
\end{array}\right)^{j}=\left(\begin{array}{c}
D^{j} \varphi_{t}(0) \\
D^{j+1} \varphi_{t}(0) \cdot \xi
\end{array}\right)\left(\begin{array}{l}
x \\
\xi
\end{array}\right)^{j}=0 \quad j=1, \ldots, k-1
$$

Segue da hipótese de indução que $D^{j} X^{\prime}(0)=0$ para $j=1, \ldots, k-1$, mas

$$
X^{\prime}\left(\begin{array}{l}
x \\
\xi
\end{array}\right)=\left(\begin{array}{c}
X(x) \\
D X(x) \cdot \xi
\end{array}\right)
$$

logo, $D^{k-1} X^{\prime}(0)=0$ implica que $D^{k} X(0)=0$, como queríamos demonstrar.

Teorema 5.4. Seja $X$ um campo de vetores $C^{k}, k=1,2, \ldots, \infty$, definido em uma vizinhança da origem em $\mathbb{R}^{s}$, com $X(0)=0$. Sejam ainda $\lambda_{1}, \ldots, \lambda_{s}$ autovalores de $D X(0)$ e suponha que eles satisfaçam a condição

$$
\lambda_{i} \neq \xi \cdot \lambda, \quad \forall \xi \in \mathbb{Z}_{+}^{s} ; 2 \leq|\xi| \leq k
$$

onde $\lambda=\left(\lambda_{1}, \ldots, \lambda_{s}\right) e^{\prime \prime}$ " representa o produto escalar usual em $\mathbb{R}^{s}$ e $|\xi|=\xi_{1}+\cdots+\xi_{s}$

Então, existe um $C^{k}$ difeormorfismo local $R$ em torno da origem, com $R(0)=0$ e $D R(0)=1$ tal que

$$
\left(R_{*} X\right) x=D X(0) x+o\left(x^{k}\right) .
$$

Demonstração: Seja $X_{0}$ o campo de vetores linear dado por $X_{0} x=D X(0) x$ e sejam $U(t)$ e $U_{0}(t)$ os fluxos locais gerados pelos campos $X$ e $X_{0}$, respectivamente. Como $X$ é $C^{k}$, segue que $U(t) \in \mathcal{S}^{k}$ para todo $t$ e como $X_{0}$ é linear então $U_{0}(t) \in \mathcal{S}^{\infty} \subset \mathcal{S}^{k}$. Sejam $\tilde{U}(t)$ e $\tilde{U}_{0}(t)$ as imagens em $\tilde{\mathcal{A}}^{k}$ de $U(t)$ e $U_{0}(t)$, respectivamente. Então, $\tilde{U}_{0}(t)$ é a parte linear de $\tilde{U}(t)$ e $U_{0}(t)=e^{t D X(0)}$. Logo, os autovalores de $U_{0}(t)$ são simplesmente $e^{t \lambda_{1}}, \ldots, e^{t \lambda_{s}} \mathrm{e}$, portanto, estamos nas hipóteses do teorema anterior. Assim, para cada $t$ 
existe $\tilde{R} \in \tilde{\mathcal{A}}^{k}$ com $D \tilde{R}(0)=I d$ tal que $\tilde{R} \tilde{U}(t) \tilde{R}^{-1}=\tilde{U}_{0}(t)$. Observe porém, que o mesmo $\tilde{R}$ que serve para $t$ funciona também para $2 t$; de fato, se $\tilde{R} \tilde{U}(t) \tilde{R}^{-1}=\tilde{U}_{0}(t)$, então

$$
\tilde{R} \tilde{U}(2 t) \tilde{R}^{-1}=\tilde{R} \tilde{U}(t) \tilde{U}(t) \tilde{R}^{-1}=\tilde{R} \tilde{U}(t) \tilde{R}^{-1} \tilde{R} \tilde{U}(t) \tilde{R}^{-1}=\tilde{U}_{0}(t) \tilde{U}_{0}(t)=\tilde{U}_{0}(2 t) .
$$

Portanto, da unicidade de $\tilde{R}$, segue que $\tilde{R}$ independe de $t$.

Temos ainda que $\mathcal{S}^{k} \rightarrow \tilde{\mathcal{A}}^{k}$ é sobrejetiva e, portanto, existe $R \in \mathcal{S}^{k}$, cuja imagem é $\tilde{R}$. Portanto, $R(0)=0, D R(0)=1 \mathrm{e}$

$$
R U(t) R^{-1} y=U_{0}(t) y+o\left(y^{k}\right)
$$

sendo $o\left(y^{k}\right)$ dependendo de $t$.

Seja, agora, $\psi(t, x)=R U(t) R^{-1} x$ e $Y=R_{*} X$, de modo que $Y$ gere o fluxo $\psi(t, x)$. Denotemos ainda $\varphi(t, x)=U_{0}(t) x$. Observe que, por construção, o polinômio de Taylor de orden $k$ de $\psi(t, x)$ e $\varphi(t, x)$ coincidem em uma vizinhança de $t_{0}=0$. Precisamos então mostrar que os campos $Y$ e $X_{0}$ possuem o mesmo polinômio de Taylor de ordem $k$ em torno de $x_{0}=0$. Mas isso segue do Lema 5.3.

Observação 5.5. Dizemos que uma matriz $M$ satisfaz a Primeira Condição de não Ressonância (CNR 1), quando seus autovalores $\lambda_{1}, \ldots, \lambda_{s}$ satisfazem a condição (5.0.3), isto é,

$$
\lambda_{i} \neq \xi \cdot \lambda, \quad \forall \xi \in \mathbb{Z}_{+}^{s} ; 2 \leq|\xi| \leq k \quad, \quad(\text { CNR 1) }
$$

onde $\lambda=\left(\lambda_{1}, \ldots, \lambda_{s}\right)$, "." representa o produto escalar usual em $\mathbb{R}^{s}$ e $|\xi|=\xi_{1}+\cdots+\xi_{s}$.

Analisemos um pouco a ideia básica por trás das demonstrações nos resultados a seguir. Sejam $U(t)$ e $U_{0}(t)$ dois grupos a um parâmetro de transformações em um espaço, por exemplo, fluxos campos de vetores em uma variedade. E suponha que exista o limite

$$
\lim _{t \rightarrow \infty} U(t) U_{0}(-t)=W
$$


note que

$$
\begin{aligned}
U(s) W U_{0}(-s) & =U(s)\left(\lim _{t \rightarrow \infty} U(t) U_{0}(-t)\right) U_{0}(-s) \\
& =\lim _{t \rightarrow \infty} U(s) U(t) U_{0}(-t) U_{0}(-s) \\
& =\lim _{t \rightarrow \infty} U(t+s) U_{0}(-t-s)=W .
\end{aligned}
$$

Se $W$ for inversível, temos então que $W^{-1} U(s) W=U_{0}(s)$ e portanto, os dois grupos a um parâmetro são conjugados. No caso particular em que eles são fluxos gerados por campos $X$ e $X_{0}$ respectivamente, isso significa que $X_{0}=W_{*}^{-1} X$.

Vamos, agora, demonstrar um lema técnico, que estabelece estimativas úteis na demonstração da existência do limite $W$.

Lema 5.6. Sejam $U \subset \mathbb{R}^{s}$ um aberto e $X: U \rightarrow \mathbb{R}^{s}$ aplicação Lipschitz, com constante de Lipschitz $k$. Seja $\varphi$ o fluxo local gerado pelo campo $X$. Então, para $x, y \in U$ e $t>0$ temos>

$$
\|\varphi(t, x)-\varphi(t, y)\| \leq e^{k t}\|x-y\| .
$$

Suponha ainda que $X=X_{0}+X_{1}$, com $X_{0}$ e $X_{1}$ localmente Lipschitz, e seja $\varphi_{0}$ o fluxo local gerado por $X_{0}$. Então, para $x \in U$ e $t>0$

$$
\left\|\varphi(t, x)-\varphi_{0}(t, x)\right\| \leq \int_{0}^{t} e^{k(t-s)}\left\|X_{1}\left(\varphi_{0}(s, x)\right)\right\| d s
$$

Demonstração: Da definição de fluxo, segue que

$$
\begin{aligned}
& \varphi(t, x)=x+\int_{0}^{t} X(\varphi(s, x)) d s \\
& \varphi(t, y)=y+\int_{0}^{t} X(\varphi(s, y)) d s .
\end{aligned}
$$

Logo,

$$
\begin{aligned}
\|\varphi(t, x)-\varphi(t, y)\| & \leq\|x-y\|+\int_{0}^{t}\|X(\varphi(s, x))-X(\varphi(s, y))\| d s \\
& \leq\|x-y\|+\int_{0}^{t} k\|\varphi(s, x)-\varphi(s, y)\| d s .
\end{aligned}
$$


Sejam, então, $f(t)=\|\varphi(t, x)-\varphi(t, y)\|$, e $\Phi$ dada por

$$
(\Phi g)(t)=\|x-y\|+\int_{0}^{t} k g(s) d s .
$$

Note que $f \leq \Phi f$. Além disso, supondo que $\Phi^{j-1} f \leq \Phi^{j} f$, temos

$$
\Phi^{j+1} f(t)-\Phi^{j} f(t)=\int_{0}^{t} k\left(\Phi^{j} f(s)-\Phi^{j-1} f(s)\right) d s \geq 0,
$$

logo, demonstramos por indução que $f \leq \Phi f \leq \Phi^{2} f \leq \Phi^{3} f \leq \ldots$

Considere ainda a equação

$$
F(t)=\|x-y\|+\int_{0}^{t} k F(s) d s
$$

que pode ser reescrita como a equação diferencial: $F^{\prime}(t)=k F(t)$, com $F(0)=\|x-y\|$. Note que, do teorema de Picard, as iteradas $\Phi^{j} f$ convergem uniformemente para uma solução desta EDO, $\log f \leq F$, mas $F$ é dada por $F(t)=e^{k t}\|x-y\|$, provando a primeira afirmação do teorema.

Para provar a segunda afirmação, consideremos

$$
\begin{aligned}
\varphi(t, x) & =x+\int_{0}^{t}\left(X_{0}+X_{1}\right)(\varphi(s, x)) d s \\
\varphi_{0}(t, x) & =x+\int_{0}^{t} X_{0}\left(\varphi_{0}(s, x)\right) d s .
\end{aligned}
$$

Então,

$$
\varphi(t, x)-\varphi_{0}(t, x)=\int_{0}^{t}\left(\left(X_{0}+X_{1}\right) \varphi(s, x)-\left(X_{0}+X_{1}\right) \varphi_{0}(s, x)\right) d s+\int_{0}^{t}\left(X_{1} \varphi(s, x)\right) d s .
$$

Logo

$$
\left\|\varphi(t, x)-\varphi_{0}(t, x)\right\| \leq \int_{0}^{t} k\left\|\varphi(s, x)-\varphi_{0}(s, x)\right\| d s+\int_{0}^{t}\left\|X_{1} \varphi(s, x)\right\| d s .
$$

De forma análoga à demonstração da afirmação anterior, vamos considerar $f(t)=$ 
$\left\|\varphi(t, x)-\varphi_{0}(t, x)\right\|$ e a aplicação $\Psi$ dada por

$$
\Psi g(t)=\int_{0}^{t}\left\|X_{1} \varphi(s, x)\right\| d s+\int_{0}^{t} k g(s) d s .
$$

Novamente, por indução, mostramos que $\Psi^{j} f \leq \Psi^{j+1} f$ para todo $j$. Consideramos ainda a equação diferencial

$$
F^{\prime}(t)=k F(t)+\left\|X_{1}\left(\varphi_{0}(t, x)\right)\right\|, \quad F(0)=0
$$

Novamente, observamos que as funções $\Psi^{j} f(f)$ são iteradas de Picard deste PVI e, portanto, do teorema de Picard, elas convergem uniformemente para a solução $F(t)=e^{k t} \int_{0}^{t} e^{-k s}\left\|X_{1}\left(\varphi_{0}(s, x)\right)\right\| d s$, finalizando a demonstração.

Vamos, agora, provar um caso particular do teorema de linearização de Sternberg. Posteriormente, usaremos isso para demonstrar o caso geral. Para auxiliar a notação, iremos denotar $f \sim g$ em $N \subseteq \mathbb{R}^{s}$ se $f-g$ é flat em $N$.

Teorema 5.7. Seja $X$ um campo de vetores $C^{\infty}$ definido em uma vizinhança da origem $0 \in \mathbb{R}^{s} \operatorname{com} X(0)=0$. Seja ainda $X_{0}=D X(0)$ e suponha que

$$
X(x)=X_{0}(x)+o\left(x^{\infty}\right)
$$

Suponha ainda que todos os autovalores $\lambda$ de $X_{0}$ satisfaçam $\mathfrak{R e} \lambda<0$. Sejam $U(t) e$ $U\left(t_{0}\right)$ os fluxos associados aos campos $X$ e $X_{0}$ respectivamente. Então,

$$
W(x)=\lim _{t \rightarrow \infty} U(-t) U_{0}(t) x
$$

existe e é um difeomorfismo $C^{\infty}$ local em torno da origem tal que

$$
W_{*}^{-1} X=X_{0}
$$

em uma vizinhança da origem.

Demonstração: Observe que só estamos interessados em campos definidos localmente, no entanto, é sempre possível estender este campo, mais ainda, é possível estendê-lo controlando os valores de sua derivada, de forma que ele seja globalmente Lipschitz. Podemos assim, sem perda de generalidade, supor que o campo $X$ é definido em todo $\mathbb{R}^{s}$ e é globalmente Lipschitz, com constante de Lipschitz $k$. Assim, o fluxo $U(t)$, 
também é globalmente definido.

Como já vimos anteriormente (Lema 4.3), o fato de que todos os autovalores $\lambda$ de $X_{0}$ possuírem parte real negativa implica que existem constante $C>0$ e $\mu>0$ tais que

$$
\left\|U_{0}(t)\right\| \leq C e^{-\mu t} ; \forall t \geq 0
$$

Defina $X_{1}$ pela equação $X=X_{0}+X_{1}$ e para $x$ fixado, seja $y=U_{0}(t) x$. Então, do Lema 5.6

$$
\begin{aligned}
\left\|U(-t) U_{0}(t) x-x\right\| & =\left\|U(-t) y-U_{0}(-t) y\right\| \leq \int_{0}^{t} e^{k(t-s)}\left\|X_{1}\left(U_{0}(-s) y\right)\right\| d s \\
& =\int_{t}^{0} e^{k r}\left\|X_{1}(\underbrace{U_{0}(r-t) y}_{U_{0}(s) \text { é um grupo }})\right\|(-d r)=\int_{0}^{t} e^{k r}\left\|X_{1}\left(U_{0}(r) U_{0}(-t) y\right)\right\| d r \\
& =\int_{0}^{t} e^{k r}\left\|X_{1}\left(U_{0}(r) x\right)\right\| d r
\end{aligned}
$$

Seja agora $t_{1}=t_{2}+t$, com $t_{1}, t_{2} \geq 0$. Novamente, pelo Lema 5.6

$$
\begin{aligned}
\left\|U\left(-t_{1}\right) U_{0}\left(t_{1}\right) x-U\left(-t_{2}\right) U_{0}\left(t_{2}\right) x\right\| & =\left\|U\left(-t_{2}-t\right) U_{0}\left(t_{2}+t\right) x-U\left(-t_{2}\right) U_{0}\left(t_{2}\right) x\right\| \\
& =\left\|U\left(-t_{2}\right) U(-t) U_{0}\left(t_{2}\right) U_{0}(t) x-U\left(-t_{2}\right) U_{0}\left(t_{2}\right) x\right\| \\
& \leq e^{k t_{2}}\left\|U(-t) U_{0}\left(t_{2}\right) U_{0}(t) x-U_{0}\left(t_{2}\right) x\right\| .
\end{aligned}
$$

Juntando as equações (5.0.4) e (5.0.7), obtemos

$$
\begin{aligned}
\left\|U\left(-t_{1}\right) U_{0}\left(t_{1}\right) x-U\left(-t_{2}\right) U_{0}\left(t_{2}\right) x\right\| & \leq e^{k t_{2}} \int_{0}^{t} e^{k s}\left\|X_{1}\left(U_{0}(s) U_{0}\left(t_{2}\right) x\right)\right\| d s \\
& =e^{k t_{2}} \int_{0}^{t} e^{k s}\left\|X_{1}\left(U_{0}\left(s+t_{2}\right) x\right)\right\| d s
\end{aligned}
$$

Por outro lado, lembrando que $X \sim X_{0}$ na origem, temos que $X_{1}$ é $o\left(x^{\infty}\right)$. Assim, 
tomando $g(x)=X_{1}(x), N=\{0\}, h(t, x)=U_{0}(t) x$ e $D(h)=\mathbb{R}^{s}$, segue do Lema 4.4 que

$$
\begin{aligned}
\left\|U\left(-t_{1}\right) U_{0}\left(t_{1}\right) x-U\left(-t_{2}\right) U_{0}\left(t_{2}\right) x\right\| & \leq e^{k t_{2}} \int_{0}^{t} e^{k s}\left\|X_{1}\left(U_{0}\left(s+t_{2}\right) x\right)\right\| d s \\
& \leq e^{k t_{2}}(\frac{K_{m} C^{m}\|x\|^{m} e^{-\mu m t_{2}}}{m \mu-k} \underbrace{\left(1-e^{(k-m \mu) t}\right)}_{<1}) \\
& \leq K_{m} C^{m}\|x\|^{m} e^{-(\mu m-k) t_{2}} \frac{1}{m \mu-k}
\end{aligned}
$$

para todo $x$ com $\|x\|<R$, para algum $R>0$ arbitrário (as constantes $K_{m}$ dependem de $R)$.

Observamos que, se $m$ for suficientemente grande $(\mu m-k>0$ ), a rede (do inglês "net") $\left(U(-t) U_{0}(t) x\right)_{t>0}$ é uma rede de Cauchy em um espaço de Banach e, portanto, o operador $W_{-}$definido por

$$
W_{-}(x)=\lim _{t \rightarrow-\infty} U(t) U_{0}(-t) x
$$

existe e está bem definido. Além disso, para $x$ em conjuntos limitados, a convergência é uniforme, de modo que $W_{-}$é contínuo.

Queremos agora provar que $W_{-}$é suave. Sejam $\psi(t, x)=D U(t) x \in \mathcal{L}\left(\mathbb{R}^{s}\right)$ e, analogamente $^{4}, \psi_{0}(t, x)=D_{x} U_{0}(t) x=U_{0}(t) \in \mathcal{L}\left(\mathbb{R}^{s}\right)$, e defina o campo $X^{\prime}: \mathbb{R}^{s} \times \mathcal{L}\left(\mathbb{R}^{s}\right) \rightarrow$ $\mathbb{R}^{s} \times \mathcal{L}\left(\mathbb{R}^{s}\right)$ da seguinte forma:

$$
X^{\prime}\left(\begin{array}{l}
x \\
\xi
\end{array}\right)=\left.\frac{d}{d t}\right|_{t=0}\left(\begin{array}{c}
\varphi(t, x) \\
\psi(t, x) \cdot \xi
\end{array}\right)=\left(\begin{array}{c}
X(\varphi(t, x)) \\
D X(\varphi(t, x)) \cdot \psi(t, x) \cdot \xi
\end{array}\right)_{t=0}=\left(\begin{array}{c}
X(x) \\
D X(x) \cdot \xi
\end{array}\right)
$$

e analogamente

$$
X_{0}^{\prime}\left(\begin{array}{c}
x \\
\xi
\end{array}\right)=\left.\frac{d}{d t}\right|_{t=0}\left(\begin{array}{c}
\varphi_{0}(t, x) \\
\psi_{0}(t, x) \xi
\end{array}\right)=\left.\frac{d}{d t}\right|_{t=0}\left(\begin{array}{c}
U_{0}(t) x \\
U_{0}(t) \cdot \xi
\end{array}\right)=\left(\begin{array}{c}
X_{0}(x) \\
X_{0} \cdot \xi
\end{array}\right)
$$

Observe que $X^{\prime}$ e $X_{0}^{\prime}$ satisfazem as hipóteses iniciais, isto é: $X^{\prime}(0)=0, X_{0}^{\prime}=D X^{\prime}(0)$ com $X^{\prime} \sim X_{0}^{\prime}$ na origem, e a parte real dos autovalores de $X_{0}^{\prime}$ é sempre estritamente

\footnotetext{
${ }^{4}$ Como $X_{0}$ é linear, segue que $U_{0}(t) x$ é linear em $x$.
} 
negativa ${ }^{5}$. Os fluxos de $X^{\prime}$ e $X_{0}^{\prime}$ são dados por

$$
\begin{aligned}
U^{\prime}(t)\left(\begin{array}{l}
x \\
\xi
\end{array}\right) & =\left(\begin{array}{c}
U(t) x \\
D U(t) x \cdot \xi
\end{array}\right) \\
U_{0}^{\prime}(t)\left(\begin{array}{l}
x \\
\xi
\end{array}\right) & =\left(\begin{array}{c}
U_{0}(t) x \\
D U_{0}(t) x \cdot \xi
\end{array}\right)=\left(\begin{array}{c}
U_{0}(t) x \\
U_{0}(t) \cdot \xi
\end{array}\right),
\end{aligned}
$$

então,

$$
U^{\prime}(-t) U_{0}^{\prime}(t)\left(\begin{array}{l}
x \\
\xi
\end{array}\right)=\left(\begin{array}{c}
U(-t)\left(U_{0}(t) x\right) \\
(D U(-t))\left(U_{0}(t) x\right) \cdot\left(U_{0}(t) \cdot \xi\right)
\end{array}\right)=\left(\begin{array}{c}
U(-t) U_{0}(t) x \\
D\left(U(-t) U_{0}(t)\right) x \cdot \xi
\end{array}\right)
$$

Mas, nos já provamos que nas condições do teorema $U^{\prime}(-t) U_{0}^{\prime}(t)$ converge, e para $(x, \xi)$ limitados, a convergência é uniforme e, portanto, o limite é contínuo. Segue então que $D\left(U(-t) U_{0}(t)\right)$ converge para uma aplicação contínua e, portanto, $W_{-}$é uma aplicação $C^{1}$. Por indução, concluímos que $W_{-} \in C^{\infty}$.

Por outro lado, fazendo $t_{2}=0$ na equação (5.0.10), segue que

$$
\left\|W_{-}(x)-x\right\| \leq \frac{K_{m}}{c m-k} C^{m}\|x\|^{m}, \forall m \in \mathbb{Z}_{+} .
$$

Logo, $W_{-}(x)=x+o\left(x^{\infty}\right)$. Portanto, $D W_{-}(0)=1$ e, consequentemente, $W_{-}$é um difeomorfismo local em torno da origem. Como observamos anteriormente, temos que $U_{0}(t)=W_{-} U(t) W_{-}^{-1}$ e, portanto, $X_{0}=\left(\left(W_{-}\right)^{-1}\right)_{*} X$.

Segue dos teoremas 5.4 e 5.7, o seguinte corolário:

Corolário 5.8. Seja $X$ um campo de vetores suave em uma vizinhança da origem em $\mathbb{R}^{s}$, com $X(0)=0$, e $D X(0)$ satisfazendo (CNR 1). Suponha ainda que cada autovalor $\lambda$ de $D X(0)$ satisfaça ainda a condição $\mathfrak{R e} \lambda<0$. Então, existe um difeomorfismo local $R$ em torno da origem, tal que $R_{*} X$ é linear em uma vizinhança da origem.

Lema 5.9. Seja $X$ um campo de vetores suave em $\mathbb{R}^{s} \operatorname{com} X(0)=0$. Sejam $X_{0}$ e $X_{1}$ os campos definido por $X_{0}(x)=D X(0) x$ e $X_{1}=X-X_{0}$ e sejam ainda $U(t)$ e $U_{0}(t)$ os fluxos gerados pelos campos $X$ e $X_{0}$, respectivamente. Suponha que, para $j=0,1,2, \ldots$, $D^{j} X$ seja globalmente Lipschitz. Suponha ainda que existem um subspaço vetorial $N$ em

\footnotetext{
${ }^{5}$ Observe que na verdade os autovalores de $X_{0}$ e $X_{0}^{\prime}$ coincidem, a menos de multiplicidade.
} 
$\mathbb{R}^{s}$ que seja invariante por $X_{0}$ e um inteiro positivo $l$ tal que, para todo $m \geq 0$ inteiro e $j=0,1,2, \ldots$, existem $\delta>0$ e $M>0$ com

$$
\|z-N\|<\delta \Rightarrow\left\|D^{j} X_{1}(z)\right\| \leq M\|z-N\|^{m}\|z\|^{l} .
$$

Seja $E$ o subspaço vetorial formado por todo $x \in \mathbb{R}^{s}$ que satisfaz

$$
\lim _{t \rightarrow \infty}\left\|U_{0}(t) x-N\right\|=0 .
$$

Então, para todo $j=0,1,2, \ldots$ e $x \in E$,

$$
D^{j}\left(U(-t) U_{0}(t)\right) x
$$

converge quando $t \rightarrow \infty$ e o limite é contínuo em $x$ para $x \in E$. Defina assim,

$$
W_{-}(x)=\lim _{t \rightarrow \infty} U(-t) U_{0}(t) x \quad ; x \in E,
$$

então, $W_{-}$possui uma extensão $C^{\infty} G: \mathbb{R}^{s} \rightarrow \mathbb{R}^{s}$ tal que $G(x)=x+o\left(x^{\infty}\right)$ em $N$ e, em uma vizinhança da origem em $E,\left(G^{-1}\right)_{*} X X_{0}$.

Demonstração: Como já vimos na demonstração do Lema 4.9, o limite

$$
\lim _{t \rightarrow \infty}\left\|U_{0}(t) x-N\right\|=0
$$

na verdade implica que existem constantes $C>0$ e $\mu>0$ tais que

$$
\left\|U_{0}(t) x-N\right\| \leq C e^{-\mu t}\|x-N\|
$$

para todo $t \geq 0, x \in E$. Seja $k$ uma constante de Lipschitz global para $X_{1}$ (essa constante existe, pois $X_{1}$ é a soma de mapas globalmente Lipschitz, em particular, podemos escolher essa constante, de modo que $k$ seja também constante de Lipschitz global para $X_{0}$ e $X$ ) e tome $m \in \mathbb{Z}_{+}$tal que $m \mu-k-l k>0$. Seja $B_{r}=\left\{x \in \mathbb{R}^{s} ;\|x-N\|<r\right\}$. Então, para todo $r>0$, existe $t_{r} \geq 0$ tal que

$$
\left\|U_{0}(s) x-N\right\| \leq \delta \quad ; \forall x \in B_{r} \cap E ; t \geq t_{r} .
$$

Observe que se $r=\frac{\delta}{C}$, então podemos tomar $t_{r}=0$. 
Seja $t_{1}=t_{2}+t, \operatorname{com} t, t_{2} \geq t_{r}$. Então, de maneira similar à demonstração anterior

$$
\begin{aligned}
\left\|U\left(-t_{1}\right) U_{0}\left(t_{1}\right) x-U\left(-t_{2}\right) U_{0}\left(t_{2}\right) x\right\| & =\left\|U\left(-t_{2}\right) U(-t) U_{0}(t) U_{0}\left(t_{2}\right) x-U\left(-t_{2}\right) U_{0}\left(t_{2}\right) x\right\| \\
& \leq e^{k t_{2}}\left\|U(-t) U_{0}(t) U_{0}\left(t_{2}\right) x-U_{0}\left(t_{2}\right) x\right\| \\
& \leq e^{k t_{2}} \int_{0}^{t} e^{k s}\left\|X_{1}\left(U_{0}\left(t_{2}+s\right) x\right)\right\| d s
\end{aligned}
$$

Lembrando que $\left\|X_{1}(z)\right\| \leq M\|z-N\|^{m}\|z\|^{l}$ se $\|z-N\|<\delta$, então para $x \in B_{r} \cap E$ e $t_{2} \geq t_{r}$

$$
\begin{aligned}
e^{k t_{2}} \int_{0}^{t} e^{k s}\left\|X_{1}\left(U_{0}\left(t_{2}+s\right) x\right)\right\| d s & \leq e^{k t_{2}} \int_{0}^{\infty} e^{k s} M\left\|U_{0}\left(t_{2}+s\right) x-N\right\|^{m}\left\|U_{0}\left(t_{2}+s\right) x\right\|^{l} d s \\
& \leq M e^{k t_{2}} \int_{0}^{\infty} e^{k s}\left(C e^{-\mu\left(s+t_{2}\right)}\|x-N\|\right)^{m}\left\|U_{0}\left(t_{2}+s\right) x\right\|^{l} d s
\end{aligned}
$$

Note que

$$
\left\|U_{0}(t) x\right\| \leq\left\|U_{0}(t)\right\|\|x\| \leq e^{\|D X(0)\| t}\|x\| \leq e^{k t}\|x\| \quad ; \quad \forall x \in E
$$

logo, para $x \in B_{r} \cap E$, e $t \geq t_{r}$ e definindo $V=V\left(t_{1}, t_{2}, x\right)=\| U\left(-t_{1}\right) U_{0}\left(t_{1}\right) x-$ $U\left(-t_{2}\right) U_{0}\left(t_{2}\right) x \|$, temos:

$$
\begin{aligned}
V & \leq M e^{k t_{2}} \int_{0}^{\infty} e^{k s}\left(C e^{-\mu\left(s+t_{2}\right)}\|x\|\right)^{m}\left\|U_{0}\left(t_{2}+s\right) x\right\|^{l} d s \\
& \leq M C^{m}\|x-N\|^{m} e^{(k-m \mu) t_{2}} \int_{0}^{\infty} e^{(k-m \mu) s}\left(e^{k\left(t_{2}+s\right)}\|x\|\right)^{l} d s \\
& \leq M C^{m} e^{(k-m \mu) t_{2}}\|x-N\|^{m} \int_{0}^{\infty} e^{(k-m \mu) s}\left(e^{k\left(t_{2}+s\right)}\|x\|\right)^{l} d s \\
& =\left(M C^{m}\|x-N\|^{m}\|x\|^{l} e^{-(m \mu-k-k l) t_{2}}\right) \frac{1}{(m \mu-k-k l)} \leq \frac{M C^{m} r^{m}\|x\|^{l} e^{-(m \mu-k-k l) t_{2}}}{(m \mu-k-k l)} \stackrel{t_{2} \rightarrow \infty}{\longrightarrow} 0
\end{aligned}
$$

Isso prova que $W_{-}$existe e, definindo $\tilde{B}_{R}=\left\{x \in \mathbb{R}^{s} ;\|x\|<R\right\}$, temos que a convergência é uniforme para $x \in \tilde{B}_{R} \cap B_{r} \cap E$. Portanto, $W_{-}$é contínua em $\tilde{B}_{R} \cap B_{r} \cap E$. Da arbitrariedade de $r$ e $R$, segue que $W_{-}$existe e é contínua em $E$. Por outro lado, se $W_{-}$ for $C^{\infty}$ (e de fato, demonstraremos isso logo adiante), então, tomando $r=\frac{\delta}{C}$, podemos fazer $t_{2}=0$ obtendo a seguinte desigualdade

$$
V\left(t_{1}, 0, x\right)=\left\|U\left(-t_{1}\right) U_{0}\left(t_{1}\right) x-x\right\| \leq \frac{M C^{m}\|x-N\|^{m}\|x\|^{l}}{(m \mu-k-k l)},
$$


para todo $x \in B_{r} \cap E$ e $t_{1} \geq 0$. Fazendo $t_{1} \rightarrow \infty$ obtemos

$$
\left\|W_{-}(x)-x\right\| \leq \frac{M C^{m}\|x-N\|^{m}\|x\|^{l}}{(m \mu-k-k l)} ; \forall x \in B_{r} \cap E
$$

e, portanto, da arbitrariedade de $m$, segue que $W_{-}$é a identidade módulo flat em $N$, isto é, $\left(W_{-}(x)-x\right)$ é $o\left(x^{\infty}\right)$ em $N$.

Para provarmos que $W_{-}$é suave, vamos repetir as contas feitas na demonstração do Teorema 5.7. Defina novamente em $\mathbb{R}^{s} \times \mathcal{L}\left(\mathbb{R}^{s}\right)$ os campos

$$
X^{\prime}\left(\begin{array}{l}
x \\
\xi
\end{array}\right)=\left(\begin{array}{c}
X(x) \\
D X(x) \cdot \xi
\end{array}\right) \text { e } X_{0}^{\prime}\left(\begin{array}{l}
x \\
\xi
\end{array}\right)=\left(\begin{array}{c}
X_{0}(x) \\
X_{0} \cdot \xi
\end{array}\right)
$$

e seja $N^{\prime} \subseteq \mathbb{R}^{s} \times \mathcal{L}\left(\mathbb{R}^{s}\right)$ o espaço de todos os $\left(\begin{array}{l}x \\ \xi\end{array}\right)$ tais que $x \in N$. Observe que $N^{\prime}$ é invariante por $X_{0}^{\prime}$. Lembrando que os fluxos de $X^{\prime}$ e $X_{0}^{\prime}$ são dados por

$$
U^{\prime}(t)\left(\begin{array}{l}
x \\
\xi
\end{array}\right)=\left(\begin{array}{c}
U(t) x \\
D U(t) x \cdot \xi
\end{array}\right) \quad e \quad U_{0}^{\prime}(t)\left(\begin{array}{l}
x \\
\xi
\end{array}\right)=\left(\begin{array}{c}
U_{0}(t) x \\
U_{0}(t) \cdot \xi
\end{array}\right)
$$

respectivamente. Defina também $X_{1}^{\prime}$ por $X^{\prime}=X_{0}^{\prime}+X_{1}^{\prime}$. Então,

$$
X_{1}^{\prime}\left(\begin{array}{l}
x \\
\xi
\end{array}\right)=\left(\begin{array}{c}
X_{1}(x) \\
D X_{1}(x) \cdot \xi
\end{array}\right)
$$

e, assim, para $(z, \eta) \in \mathbb{R}^{s} \times \mathcal{L}\left(\mathbb{R}^{s}\right)$ temos:

$$
\left\|D^{j} X_{1}^{\prime}(z, \eta)\right\| \leq\left\|D^{j} X_{1}(z)\right\|+\left\|D^{j+1} X_{1}(z) \cdot \eta\right\| \leq\left\|D^{j} X_{1}(z)\right\|+\left\|D^{j+1} X_{1}(z)\right\|\|(z, \eta)\| .
$$

Por hipótese, para cada $m$ e para cada $j$ existem $\delta_{0}, \delta_{1}, M_{0}, M_{1}$ tais que $\left\|D^{j} X_{1}(z)\right\| \leq$ $M_{0}\|z-N\|^{m}\|z\|^{l}$ e $\left\|D^{j+1} X_{1}(z)\right\| \leq M_{1}\|z-N\|^{m}\|z\|^{l}$ se $\|z-N\|$ for menor que $\delta_{0}$ e $\delta_{1}$ respectivamente. Tomando $\delta=\min \left\{\delta_{0}, \delta_{1}\right\}$ então $\left\|(z, \eta)-N^{\prime}\right\|=\|z-N\|<\delta$ implica

$$
\begin{aligned}
\left\|D^{j} X_{1}(z, \eta)\right\| & \leq M_{0}\|z-N\|^{m}\|z\|^{l}+M_{1}\|z-N\|^{m}\|z\|^{l}\|(z, \eta)\| \\
& \leq M\|z-N\|^{m}\|(z, \eta)\|^{l+1}=M\left\|(z, \eta)-N^{\prime}\right\|^{m}\|(z, \eta)\|^{l+1} .
\end{aligned}
$$


Finalmente, observando que

$$
\lim _{t \rightarrow \infty}\left\|U_{0}^{\prime}(t)(x, \xi)-N^{\prime}\right\|=0 \Leftrightarrow \lim _{t \rightarrow \infty}\left\|U_{0}(t) x-N\right\|=0
$$

temos que se $E^{\prime} \subseteq \mathbb{R}^{s} \times \mathcal{L}\left(\mathbb{R}^{s}\right)$ é o espaço formado por todos os $\left(\begin{array}{l}x \\ \xi\end{array}\right)$ tais que $x \in E$, então, $X^{\prime}$ e $X_{0}^{\prime}$ estão nas condições do teorema e neste caso concluímos que

$$
U^{\prime}(-t) U_{0}^{\prime}(t)\left(\begin{array}{l}
x \\
\xi
\end{array}\right)=\left(\begin{array}{c}
U(-t) U_{0}(t) x \\
D\left(U(-t) U_{0}(t)\right) x \cdot \xi
\end{array}\right)
$$

converge a uma função contínua para todo $x \in E$. Portanto, $D\left(U(-t) U_{0}(t)\right)$ converge, quando $t \rightarrow \infty$, a uma função contínua em $x$, para todo $x \in E$. Mostramos assim que $W_{-}$é $C^{1}$ em $E$. Por indução, mostramos que $D^{j}\left(U(-t) U_{0}(t)\right)$ converge a uma função contínua de $x \in E$. De modo que $W_{-}$é suave.

Precisamos, agora, encontrar uma maneira de estender (suavemente) $W_{-}$a uma aplicação $G: \mathbb{R}^{s} \rightarrow \mathbb{R}^{s}$ que é a identidade modulo flat em uma vizinhança da origem em $N$. Considere as seguintes aplicações

$$
G_{j}(x)=\frac{1}{j !} \lim _{t \rightarrow \infty} D^{j}\left(U(-t) U_{0}(t)\right) x
$$

para cada $x \in E$. Como já vimos, $G(x)$ está bem definida. Seja agora $\rho: \mathbb{R} \rightarrow \mathbb{R}$ uma função suave, positiva, de suporte compacto tal que $0 \leq \rho \leq 1, \rho \equiv 1$ em uma vizinhança da origem, $\operatorname{e} \operatorname{supp}(\rho) \subseteq[-1,1]$. Seja ainda $E^{\perp}$ o complemento ortogonal de $E$, de modo que $\mathbb{R}^{s}=E \oplus E^{\perp}$. Agora, de maneira análoga à demonstração de 4.11, defina

$$
\tilde{G}(x \oplus y)=\sum_{j=0}^{\infty} G_{j}(x) y^{j} \rho\left(\left\|G_{j}(x)\right\|^{2}\|y\|_{E^{\perp}}^{2}\right),
$$

onde $\|y\|_{E^{\perp}}=\|y-E\|$ e $\left\|G_{j}(x)\right\|$ é a norma euclidiana usual. Essa função não está definida sempre, mas ela é bem definida em uma vizinhança $B$ de $E$. De fato, observe que se $\left\|G_{j}(x)\right\|^{2}\|y\|^{2} \geq 1$, então o $j=$ ésimo termo da série se anula. Assim, existe uma subsequencia de $\mathbb{Z}_{+}$, denotada por $\left(j_{n}\right)_{n \in \mathbb{Z}_{+}}$tal que

$$
\tilde{G}(x \oplus y)=\sum_{n=0}^{\infty} G_{j_{n}}(x) y^{j_{n}} \rho\left(\left\|G_{j_{n}}(x)\right\|^{2}\|y\|_{E^{\perp}}^{2}\right)
$$


com $\left\|G_{j_{n}}(x)\right\|\|y\|_{E^{\perp}} \leq 1$ para todo $n \in \mathbb{Z}_{+}$. Assim,

$$
\begin{aligned}
& \|\tilde{G}(x \oplus y)\| \leq \sum_{n=0}^{\infty}\left\|G_{j_{n}}(x)\right\|\|y\|_{E^{\perp}}^{j_{n}} \rho\left(\left\|G_{j_{n}}\right\|\|y\|_{E^{\perp}}^{2}\right) \\
& \leq \sum_{n=0}^{\infty}\left\|G_{j_{n}}(x)\right\|\|y\|_{E^{\perp}}^{j_{n}} \\
& =\left\|G_{j_{0}}(x)\right\|\|y\|_{E^{\perp}}^{j_{0}}+\sum_{n=1}^{\infty} \underbrace{\left\|G_{j_{n}}(x)\right\|\|y\|_{E^{\perp}} \|}_{<1}\|y\|_{E^{\perp}}^{j_{n}-1}<\left\|G_{j_{0}}(x)\right\|\|y\|_{E^{\perp}}^{j_{0}}+\sum_{n=1}^{\infty}\|y\|_{E^{\perp}}^{j_{n}-1}
\end{aligned}
$$

Definindo $B=\left\{x \oplus y \in E \oplus E^{\perp} \equiv \mathbb{R}^{s} ;\|y-E\|<r<1\right\}$, temos que a soma acima converge uniformemente em $B$ e, portanto, $\tilde{G}(x \oplus y)$ está bem definida em $B$. Além disso, nesta vizinhança de $E$, temos que $\tilde{G}$ satisfaz as seguintes propriedades:

- $\tilde{G}$ é $C^{\infty}$. Para provar isso, derivamos a série termo a termo e repetimos a conta acima, para provar que e série das derivadas é uniformemente convergente em em B.

- $D^{j} \tilde{G}(x)=j ! G_{j}(x)$ para todo $x \in E$. Em particular, $D^{j}(\tilde{G}(x)-x)=0$ para todo $x \in N$, de modo que $G$ é a identidade módulo flat em $N$.

- $\tilde{G}$ é uma extensão suave de $W_{-}$.

Escolhendo uma função suave $\alpha: \mathbb{R}^{s} \rightarrow R$ com suporte nessa vizinhança e tal que $\alpha \equiv 1$ em uma vizinhança (um pouco menor) de $E$, podemos definir $G=\alpha \tilde{G}$. Observemos que, como $\alpha$ vale um em uma vizinhança de $E$, então $G$ possui as mesmas propriedades de $\tilde{G}$ listadas acima.

Como $G$ é uma extensão de $W_{-}$, segue que $U(-t) G U_{0}(t) x=G(x)$, para $x$ em uma vizinhança de 0 em $E$. Além disso, para cada $s$ fixado, temos que

$$
D^{j}\left(U(-s) U(-t) U_{0}(t) U_{0}(s)\right) x=D^{j}\left(U(-t-s) U_{0}(t+s)\right) x \underset{t \rightarrow \infty}{\longrightarrow} D^{j} G(x),
$$

mas

$$
D^{j}\left(U(-s) U(-t) U_{0}(t) U_{0}(s)\right) x \underset{t \rightarrow \infty}{\longrightarrow} D^{j}\left(U(-s) G U_{0}(s)\right)(x) .
$$

Logo, $U(-s) G U_{0}(s)=G$ módulo flat em uma vizinhança da origem em $E$. Assim, $U_{0}(s)=G U(s) G^{-1}$ em $E$ a menos de uma função flat. Segue do Lema 5.3 que 
$\left(G^{-1}\right)_{*} X=X_{0}+o\left(x^{\infty}\right)$ em uma vizinhança da origem em $E$

Finalmente, vamos ao teorema de linearização de Sternberg

Teorema 5.10. Seja $X$ um campo suave em uma vizinhança da origem $0 \in \mathbb{R}^{s}$, com $X(0)=0$. Suponha que $D X(0)$ satisfaça a condição (CNR 1). Então, existe um difeomorfismo local $F$ em torno da origem, tal que $F_{*} X$ é linear em uma vizinhança da origem.

Demonstração: Segue do Teorema 5.4, que existe um difeomorfismo local $R$ tal que $R_{*} X=D X(0)+o\left(x^{\infty}\right)$, de modo que podemos, sem perda de generalidade assumir que $X \sim X_{0}$ na origem, onde $X_{0}(x)=D X(0) x$.

Seja $U_{0}(t)$ o fluxo associado ao campo $X_{0}$ e considere $E^{-}$e $E^{+}$os auto-espaços de $\mathbb{R}^{s}$ associados aos autovalores com partes reais negativas e positivas (respcetivamente) de $X_{0}$, de modo que

$$
x \in E^{-} \Leftrightarrow \lim _{t \rightarrow \infty} U_{0}(t) x=0 \quad ; \quad x \in E^{+} \Leftrightarrow \lim _{t \rightarrow \infty} U_{0}(-t) x=0 .
$$

Seja $f \in C_{c}^{\infty}\left(\mathbb{R}^{s}\right)$, com $f \equiv 1$ em uma vizinhança da origem e com suporte dentro da vizinhança onde $X$ está definida. Seja $X^{\prime}=-f X$. Como $X^{\prime}$ tem suporte compacto, segue que $D^{j} X^{\prime}$ é globalmente Lipschitz. Tomando $N=\{0\}$, temos que $N$ é invariante por $-X_{0}=D X^{\prime}(0)$. Além disso, como $X_{1}=X^{\prime}-D X^{\prime}(0) \sim 0$ na origem, segue que $X_{1}$ satisfaz a estimativa (5.0.13). Finalmente, tomando $E=E^{+}$, temos que

$$
\lim _{t \rightarrow \infty}\left\|U_{0}(-t) x-N\right\|=0 \Leftrightarrow x \in E
$$

Estamos, então, em condições de aplicar o Lema 5.9 e concluimos que existe um difeomeorfismo local $G$, tal que $G_{*} X^{\prime}-D X^{\prime}(0)$ é flat em uma vizinhança da origem em $E$. Vamos, novamente sem perda de generalidade, supor que este seja o caso. Isto é, $X$ é um campo suave em torno da origem, com $X \sim D X(0)$ em uma vizinhança $B$ da origem em $E^{+}$.

Seja $\alpha: \mathbb{R}^{s} \rightarrow \mathbb{R}$ uma função suave de suporte compacto tal que $\alpha \equiv 1$ em uma vizinhança da origem e $\operatorname{supp}(\alpha) \cap E^{+} \subseteq B$.

Seja $\tilde{X}=\left(X_{0}+\alpha\left(X-X_{0}\right)\right) f$, onde $f \in C_{c}^{\infty}\left(\mathbb{R}^{s}\right)$ é como vimos anteriormente. Temos 
que $\tilde{X}=X_{0}$ fora de um compacto e $\tilde{X} \sim X_{0}$ em $E^{+}$. Observe que $D \tilde{X}(0)=X_{0}$. Além disso, como $\tilde{X}$ tem suporte compacto, então $D^{j} \tilde{X}$ é globalmente Lipschitz para todo $j \in \mathbb{Z}_{+}$.

Considere $N=E^{+}$. Temos que $N$ é invariante por $X_{0}$. Definindo $X_{1}$ por $X_{1}=\tilde{X}-X_{0}$, temos que $X_{1}$ é flat em $N$ e, portanto, $X_{1}$ satisfaz a estimativa (5.0.13). Além disso, escrevendo $x \oplus y \in E^{-} \oplus E^{+}=R^{s}$, temos

$$
\left\|U_{0}(t)(x \oplus y)-N\right\|=\|U_{0}(t)(x)+\underbrace{U_{0}(t)(y)-N}_{\in N}\|=\left\|U_{0}(t)(x)+N\right\| .
$$

Como $U_{0}(t) x \stackrel{t \rightarrow \infty}{\longrightarrow} 0$, para todo $x \in E^{-}$e $0 \in N$ temos

$$
\left\|U_{0}(t)(x \oplus y)-N\right\|=\left\|U_{0}(t)(x)+N\right\| \leq\left\|U_{0}(t) x\right\| \rightarrow 0 ; \forall x \oplus y \in E^{-} \oplus E^{+}=R^{s} .
$$

Logo, tomando $E=\mathbb{R}^{s}$, podemos aplicar o Lema 5.9 obtendo que existe um difeomorfismo local $\tilde{G}$ em torno da origem, tal que $\tilde{G}_{*} \tilde{X} \sim X_{0}$ em uma vizinhança da origem em $E=\mathbb{R}^{s}$ e, em particular, que nesta vizinhança $\tilde{G}_{*} \tilde{X}=X_{0}$. Como $\tilde{X}=X$ em uma vizinhança (possivelmente menor) da origem, segue o resultado. 


\section{Capítulo}

Resolubilidade local de campos vetoriais reais com singularidade isolada

\subsection{Resultado Principal}

Seja $L$ um campo vetorial com coeficientes lineares reais e $c$ uma constante arbitrária. O teorema que apresentamos do Guillemin-Schaeffer estabelece condições para que a equação $(L+c) u=f$ admita solução $u \in C^{\infty}\left(\mathbb{R}^{s}\right)$ quando $f$ é flat em torno da origem; a condição de que $f$ seja flat é bastante restritiva, limitando o alcance do teorema. Gostariamos, então, de estender o resultado, e obter condições para que $(L+c) u=f$ seja localmente resolúvel para $f \in C^{\infty}\left(\mathbb{R}^{s}\right)$. O resultado final que demonstraremos nesta sessão (obtido em [Silva, dos Santos Filho, 2006]) vai além, e, considerando o caso em que $c$ não é mais constante (e sim uma função em $C^{\infty}\left(\mathbb{R}^{s}\right)$ ), estabelece uma condição de não ressonância adicional em $c(0)$, que garante a resolubilidade local de $L+c$. A demonstração que apresentaremos segue de perto a demonstração feita por Fronza em sua tese de doutorado [Silva, 2006].

Lema 6.1. Seja $L$ um campo vetorial real em $\mathbb{R}^{s}$ e seja $x_{0}=0$ um ponto crítico isolado de $L$. Sejam $\lambda_{1}, \ldots, \lambda_{s^{\prime}}, \lambda_{s^{\prime}+1}, \ldots, \lambda_{s}$ os autovalores de $D L(0)$, onde $\lambda_{1}, \ldots, \lambda_{s^{\prime}}$ são os autovalores reais e $\lambda_{s^{\prime}+1}, \ldots, \lambda_{s}$ são os autovalores com parte imaginárias não nulas de $D L(0)$, sendo $\lambda_{s^{\prime}+2 j-1}$ e $\lambda_{s^{\prime}+2 j}$ autovalores conjugados. Seja ainda $c \in C^{\infty}\left(\mathbb{R}^{s}\right)$. Então, são equivalentes: 
(a): c satisfaz a seguinte condição de não ressonância (denotada por (CNR 2)),

$$
-c(0) \neq \sum_{j=1}^{s^{\prime}} m_{j} \lambda_{j}+2 \sum_{l=1}^{\left(s-s^{\prime}\right) / 2} m_{l}^{\prime} \mathfrak{R e}\left(\lambda_{s^{\prime}+2 l}\right)+\sum_{l=1}^{\left(s-s^{\prime}\right) / 2} i K(l) \mathfrak{I m}\left(\lambda_{s^{\prime}+2 l}\right)
$$

$\operatorname{com} m_{j}, m_{l}^{\prime} \in \mathbb{Z}_{+}, K(l) \in \mathcal{M}\left(m_{l}\right)$ e $\mathcal{M}(m)=\{-m, 2-m, \ldots, m-2, m\}$

(b): Para todo $f \in C^{\infty}\left(\mathbb{R}^{s}\right)$ existe $u \in C^{\infty}\left(\mathbb{R}^{s}\right)$ tal que $(L+c) u-f$ é uma função flat na origem.

Demonstração: Considere $L=\sum_{j=1}^{s} a_{j}(x) \frac{\partial}{\partial x^{j}}$ e denote $P=L+c$. Temos que $P u \sim f$ na origem, significa que todas as derivadas, de todas as ordens de $P u$ e de $f$ coincidem. Para evitar que a notação fique carregada, escreveremos $\partial^{\alpha}=\frac{\partial^{|\alpha|}}{\partial x^{\alpha}}$. Assim,

$$
P u \sim f \text { na origem } \Leftrightarrow \partial^{\alpha}(P u)(0)=\partial^{\alpha} f(0) \quad \forall \alpha \in \mathbb{Z}_{+}^{s}
$$

Temos

$$
\begin{aligned}
\partial^{\alpha}(L u)(0) & =\sum_{j=1}^{s} \partial^{\alpha}\left(a_{j} \partial^{e_{j}} u\right)(0)=\sum_{j=1}^{s} \sum_{\beta \leq \alpha}\left(\begin{array}{c}
\alpha \\
\beta
\end{array}\right)\left(\partial^{\beta} a_{j}(0) \partial^{e_{j}+\alpha-\beta} u(0)\right) \\
& =R_{1}^{\alpha}+\sum_{j=1}^{s} \sum_{k=1}^{s}\left(\begin{array}{c}
\alpha \\
e_{k}
\end{array}\right)\left(\partial^{e_{k}} a_{j}(0) \partial^{\alpha+e_{j}-e_{k}} u(0)\right)+\sum_{j=1}^{s} a_{j}(0) \partial^{e^{j}+\alpha} u(0) \\
& =R_{1}^{\alpha}+\sum_{j=1}^{s} \sum_{k=1}^{s} \alpha_{k} \partial^{e_{k}} a_{j}(0) \partial^{\alpha+e_{j}-e_{k}} u(0)
\end{aligned}
$$

onde

$$
R_{1}^{\alpha}=\sum_{j=1}^{s} \sum_{\substack{\beta \leq \alpha \\
|\beta|>1}}\left(\begin{array}{c}
\alpha \\
\beta
\end{array}\right)\left(\partial^{\beta} a_{j}(0) \partial^{e_{j}+\alpha-\beta} u(0)\right)
$$

Similarmente, temos

$$
\partial^{\alpha}(c u)(0)=\sum_{\gamma \leq \alpha}\left(\begin{array}{l}
\alpha \\
\gamma
\end{array}\right)\left(\partial^{\alpha-\gamma} c(0) \partial^{\gamma} u(0)\right)=c(0) \partial^{\alpha} u(0)+R_{2}^{\alpha},
$$

onde

$$
R_{2}^{\alpha}=\sum_{\gamma<\alpha}\left(\begin{array}{c}
\alpha \\
\gamma
\end{array}\right)\left(\partial^{\alpha-\gamma} c(0) \partial^{\gamma} u(0)\right)
$$


Assim, temos $P u \sim f$ se, e somente se,

$$
\sum_{j=1}^{s} \sum_{k=1}^{s} \alpha_{k} \partial^{e_{k}} a_{j}(0) \partial^{\alpha+e_{j}-e_{k}} u(0)+c(0) \partial^{\alpha} u(0)+R_{1}^{\alpha}+R_{2}^{\alpha}=\partial^{\alpha} f(0)
$$

para todo $\alpha \in \mathbb{Z}_{+}^{s}$. Observe que $R_{1}^{\alpha}$ e $R_{2}^{\alpha}$ só dependem das derivadas de $u$ de ordem menor ou igual a $|\alpha|-1$. Defina $\Lambda_{s}^{m}=\left\{\alpha \in \mathbb{Z}_{+}^{s} ;|\alpha|=m\right\}$ e $M=\sharp \Lambda_{s}^{m}$. Fixe uma ordenação em $\Lambda_{s}^{m}$, digamos $\left(\alpha^{1}, \alpha^{2}, \ldots, \alpha^{M}\right)$. Denote $\partial^{m}=\left(\partial^{\alpha^{1}} u(0), \ldots, \partial^{\alpha^{M}} u(0)\right)$ e $f^{m}=\left(\partial^{\alpha^{1}} f(0), \ldots, \partial^{\alpha^{M}} f(0)\right)$. Podemos reescrever (6.1.2) na forma

$$
\left(B^{m}+c(0) I\right) \partial^{m}=f^{m}+v^{m-1},
$$

para todo $m \in \mathbb{Z}_{+}$, sendo $B^{m}$ uma matriz quadrada de ordem $M$, cujas entradas dependem de $D L(0)$ e da ordenação de $\Lambda_{s}^{m}$ e $v^{m-1} \in \mathbb{C}^{M}$ satisfaz $^{1}$

$$
\left\{\partial^{\alpha} u(0)=0 \forall \alpha \in \mathbb{Z}_{+}^{s} \operatorname{com}|\alpha| \leq m-1\right\} \Rightarrow v^{m-1}=0
$$

Lema 6.2. Existe uma ordenação de $\Lambda_{s}^{m}$ tal que

$\operatorname{Spec} B^{m}=\left\{\sum_{j=1}^{s^{\prime}} m_{j} \lambda_{j}+2 \sum_{l=1}^{\left(s-s^{\prime}\right) / 2} m_{l}^{\prime} \mathfrak{R e}\left(\lambda_{s^{\prime}+2 l}\right)+\sum_{l=1}^{\left(s-s^{\prime}\right) / 2} i K(l) \mathfrak{I m}\left(\lambda_{s^{\prime}+2 l}\right), K(l) \in \mathcal{M}\left(m_{l}^{\prime}\right)\right\}$, sendo $m_{1}+\ldots m_{s}^{\prime}+m_{1}^{\prime}+m_{\left(s-s^{\prime}\right) / 2}^{\prime}=m$ e $\mathcal{M}(m)=\{-m,-m+2, \ldots, m-2, m\}$

Suponha que este lema seja verdadeiro. Temos que, se vale a condição (CNR 2), então (6.1.3) pode ser reescrito da seguinte forma:

$$
\partial^{m}=\left(B^{m}+c(0) I\right)^{-1}\left(f^{m}+v^{m-1}\right),
$$

assim, é possivel construir, a partir dos valores (determinados unicamente e indutivamente em $m$ pelos sistemas (6.1.3)) $\partial^{\alpha}(u)$, uma função suave $u$, que satisfaz (b).

Reciprocamente, suponha que (a) não seja válido. Então, $c(0) \in \operatorname{Spec} B^{m_{0}}$ para algum $m_{0} \in \mathbb{Z}_{+}$. Suponhamos $m_{0}$ minimal para esta propriedade. Vamos provar que existe $f \in C^{\infty}\left(\mathbb{R}^{s}\right)$ tal que o sistema (6.1.3) não possui solução (e, portanto, não é possível construir a $u$ desejada). Se $m_{0}=0$, basta tomar $f \operatorname{com} f(0) \neq 0$. Se $m_{0}>0$ então, como

\footnotetext{
${ }^{1}$ Para $m=0$, não existem derivadas de ordem menor e, portanto, definimos $v^{-1}=0 \in \mathbb{R}$.
} 
$c(0) \in \operatorname{Spec} B^{m_{0}}$, segue que existe $w \in \mathbb{C}^{M_{0}}$ tal que:

$$
\left(B^{m_{0}}+c(0) I\right) u^{m_{0}} \neq w ; \forall u^{m_{0}} \in \mathbb{C}^{M_{0}} .
$$

Basta escolher, então, $f \in C^{\infty}\left(\mathbb{R}^{s}\right)$ tal que $\partial^{\alpha} f(0)=0$ se $|\alpha|<m_{0}$ e $f^{m_{0}}=w$. Segue de (6.1.4) que

$$
\left(B^{m_{0}}+c(0) I\right) u^{m_{0}} \neq f^{m_{0}}+v^{m_{0}-1} ; \forall u^{m_{0}} \in \mathbb{C}^{M_{0}} .
$$

Resta, agora, demonstrar o Lema 6.2. Isso será feito na próxima sessão

Teorema 6.3. Considere o operador $P=L+c$, sendo $L$ um campo vetorial com singularidade isolada na origem e c uma função qualquer. Suponha que as condições de não ressonância (CNR 1) e (CNR 2) sejam satisfeitas. Então, para todo $f \in C^{\infty}\left(\mathbb{R}^{s}\right)$, existe $u \in C^{\infty}\left(\mathbb{R}^{s}\right)$ tal que $P u=f$ em uma vizinhança da origem.

Lembrando que $L$ satisfaz a condição de não ressonância (CNR 1) se os autovalores $\lambda_{1}, \ldots, \lambda_{s}$ de $D L(0)$ satisfazem

$$
\lambda_{i} \neq \xi \cdot \lambda, \quad \forall \xi \in \mathbb{Z}_{+}^{s} ; 2 \leq|\xi| \leq k \quad, \quad(\text { CNR 1) }
$$

onde $\lambda=\left(\lambda_{1}, \ldots, \lambda_{s}\right)$.

Demonstração: Basta provarmos que, dada $g \in C^{\infty}\left(\mathbb{R}^{s}\right)$ flat na origem, existe $u \in C^{\infty}\left(\mathbb{R}^{s}\right)$ tal que $P u=g$. De fato, sabemos do Lema 6.1 que, nas condições do teorema, dada $f \in C^{\infty}\left(\mathbb{R}^{s}\right)$, existe $v \in C^{\infty}\left(\mathbb{R}^{s}\right)$ tal que $g=f-P v$ é uma função flat. Assim $P(u+v)=P u+P v=g+(f-g)=f$.

A condição da não ressonância (CNR 2) implica que $c(0) \neq 0$. Assim, $P_{1}=\frac{1}{c} P$ está bem definido em uma vizinhança da origem. Temos que $P_{1}=L_{1}+1$, sendo $L_{1}=\frac{1}{c} L$. Além disso,

$$
D L_{1}(0)=\frac{1}{c(0)} D L(0)+\left.D\left(\frac{1}{c(x)}\right)\right|_{x=0} L(0) \text {. }
$$

Como $L(0)=0$, segue que

$$
D L_{1}(0)=\frac{1}{c(0)} D L(0) .
$$


Então, se $\lambda_{1}, \ldots, \lambda_{s}$ são autovalores de $D L(0)$, temos que $\tilde{\lambda}_{i}=\frac{1}{c(0)} \lambda_{i}$ é autovalor de $D L_{1}(0)$. Logo, $L_{1}$ também satisfaz a condição de não ressonância (CNR 1). O teorema de Sternberg nos garante que existe uma mudança de coordenadas $F$, tal que

$$
P_{2}=F_{*} P_{1}=\frac{1}{c_{0}} D L(0)+1 .
$$

Segue do teorema do Guillemin-Schaeffer (Teorema 4.11) que, dada $g \in C^{\infty}\left(\mathbb{R}^{s}\right)$ flat na origem, existe $u \in C^{\infty}\left(\mathbb{R}^{s}\right)$ tal que $P_{2} u=g$, como queríamos.

\subsection{Demonstração do Lema 6.2}

\subsubsection{Sobre ordenação de conjuntos}

Definição 6.4. Seja $\Lambda$ um conjunto finito, com $|\Lambda|=\sharp \Lambda$. Uma ordenação de $\Lambda$ é uma bijeção $h:\{1,2, \ldots,|\Lambda|\} \rightarrow \Lambda$. Dizemos que $h(k)$ sucede $h(j)$ quando $j<k$. Dizemos ainda que $h(j+1)$ é o sucessor de $h(j)$. Quando $k_{1}<j<k_{2}$ ou $k_{2}<j<k_{1}$ dizemos que $h(j)$ está entre $h\left(k_{1}\right)$ e $h\left(k_{2}\right)$.

Definição 6.5. Dados dois conjuntos finitos $\Lambda_{1}$ e $\Lambda_{2}$, com ordenações $h_{1}$ e $h_{2}$ respectivamente, definimos em $\Lambda_{1} \times \Lambda_{2}$ a ordenação produto $h_{1} \times h_{2}$ da seguinte forma. Para cada $k=1,2, \ldots,\left|\Lambda_{1}\right|$, defina

$$
A_{k}=\{(k-1)\left|\Lambda_{2}\right|+1,(k-1)\left|\Lambda_{2}\right|+2, \ldots, \underbrace{(k-1)\left|\Lambda_{2}\right|+\left|\Lambda_{2}\right|}_{k\left|\Lambda_{2}\right|}\} .
$$

Note que $k_{1} \neq k_{2}$ implica que $A_{k_{1}}$ e $A_{k_{2}}$ são disjuntos, mais precisamente, se $n \in A_{k_{1}}$ e $m \in A_{k_{2}}$, então $k_{1}<k_{2} \Rightarrow n<m$. Além disso,

$$
A_{1} \cup A_{2} \cup \cdots \cup A_{\left|\Lambda_{1}\right|}=\left\{1, \ldots,\left|\Lambda_{1}\right|\left|\Lambda_{2}\right|\right\}=\left\{1, \ldots,\left|\Lambda_{1} \times \Lambda_{2}\right|\right\} .
$$

Definimos, então,

$$
\left(h_{1} \times h_{2}\right)(j)=\left(h_{1}(k), h_{2}\left(j-(k-1)\left|\Lambda_{2}\right|\right)\right) ; \quad \text { se } j \in A_{k} .
$$

Note que se $\alpha_{1}=h_{1}\left(k_{1}\right)$ e $\alpha_{2}=h_{1}\left(k_{2}\right) \in \Lambda_{1}$ são tais que $\alpha_{2}$ sucede $\alpha_{1}$, então $\left(\alpha_{2}, \beta\right)$ sucede $\left(\alpha_{1}, \beta\right)$. De fato, escrevendo $\left(\alpha_{2}, \beta\right)=\left(h_{1} \times h_{2}\right)(m)$ e $\left(\alpha_{1}, \beta\right)=\left(h_{1} \times h_{2}\right)(n)$, temos que $m \in A_{k_{2}}$ e $n \in A_{k_{1}}$. Como $\alpha_{2}$ sucede $\alpha_{1}$, temos $k_{1}<k_{2}$ e, portanto, $n<m$. 
Além disso, entre $\left(\alpha_{2}, \beta\right)$ e $\left(\alpha_{1}, \beta\right)$, existem exatamente, $\left(k_{2}-k_{1}\right)\left|\Lambda_{2}\right|-1$ elementos. De fato, se $\beta=h_{2}(l)$, então

$$
\left\{\begin{array}{ll}
\left(\alpha_{1}, \beta\right)=\left(h_{1} \times h_{2}\right)(n)= & \left.\left(h_{1} \times h_{2}\right)\left(\left(k_{1}-1\right)\left|\Lambda_{2}\right|+l\right)\right) \\
\left(\alpha_{2}, \beta\right)=\left(h_{1} \times h_{2}\right)(m)= & \left.\left(h_{1} \times h_{2}\right)\left(\left(k_{2}-1\right)\left|\Lambda_{2}\right|+l\right)\right)
\end{array} .\right.
$$

Portanto, entre $\left(\alpha_{2}, \beta\right)$ e $\left(\alpha_{1}, \beta\right)$, existem exatamente $m-n-1=\left(k_{2}-k_{1}\right)\left|\Lambda_{2}\right|-1$ elementos. Em particular, se $k_{2}$ é o sucessor de $k_{1}$, então entre $\left(\alpha_{2}, \beta\right)$ e $\left(\alpha_{1}, \beta\right)$ existem exatamente $\left|\Lambda_{2}\right|-1$ elementos. É simples verificar também que a ordenação produto é associativa no seguinte sentido: se $\Lambda_{1}, \Lambda_{2}$ e $\Lambda_{3}$ são conjuntos finitos com ordenações $h_{1}$, $h_{2}$ e $h_{3}$ respectivamente, então $\left(\left(h_{1} \times h_{2}\right) \times h_{3}\right)(j)=\left(h_{1} \times\left(h_{2} \times h_{3}\right)\right)(j)$, para qualquer $j \in\left\{1, \ldots,\left|\Lambda_{1} \times \Lambda_{2} \times \Lambda_{3}\right|\right\}$; assim, faz sentido falar da ordem produto de um número finito de conjuntos ordenados (também finitos) qualquer.

Considere, agora, $\Lambda_{s}^{m}=\left\{\alpha \in \mathbb{Z}_{+}^{s} ;|\alpha|=m\right\}$. Defina neste conjunto a ordem lexicográfica usual ${ }^{2}$. Então, $\Lambda_{s}^{m}$ munido desta ordem satisfaz:

P0: $\forall \alpha \in \Lambda_{s}^{m}$, temos que $\alpha+e_{j}-e_{k}$ sucede $\alpha$ se $j<k$ e $\alpha_{k} \neq 0$.

Em $\Lambda_{2}^{m}$ a ordenação lexicográfica é escrita de maneira explicita como:

$$
h(j+1)=(j, m-j) ; j=0,1,2, \ldots, m .
$$

Dados $m_{1}, \ldots, m_{n} \in \mathbb{Z}_{+}$, considere o conjunto $\Lambda_{2}^{m_{1}} \times \cdots \times \Lambda_{2}^{m_{n}}$ com a ordenação produto. Então, das observações que fizemos acerca da ordenação produto obtemos a seguinte propriedade:

P1: Se $\alpha \in \Lambda_{2}^{m_{1}} \times \cdots \times \Lambda_{2}^{m_{n}}$ e $\alpha_{2 k} \neq 0, k=1, \ldots, n$, então, $\alpha+e_{2 k-1}-e_{2 k}$ sucede $\alpha$ e entre $\alpha$ e $\alpha+e_{2 k-1}-e_{2 k}$ existem exatamente $\left|\Lambda_{2}^{m_{k}+1} \times \cdots \times \Lambda_{2}^{m_{n}}\right|-1$ elementos.

Analogamente, temos

P1': Se $\alpha \in \Lambda_{2}^{m_{1}} \times \cdots \times \Lambda_{2}^{m_{n}}$ e $\alpha_{2 k_{-} 1} \neq 0, k=1, \ldots, n$, então, $\alpha$ sucede $\alpha-e_{2 k-1}+e_{2 k}$ e entre $\alpha$ e $\alpha-e_{2 k-1}+e_{2 k}$ existem exatamente $\left|\Lambda_{2}^{m_{k}+1} \times \cdots \times \Lambda_{2}^{m_{n}}\right|-1$ elementos.

Em particular, se $\alpha_{2 n} \neq 0$, então $\alpha+e_{2 n-1}-e_{2 n}$ é o sucessor de $\alpha$ e se $\alpha_{2 n-1} \neq 0$ então $\alpha$ é o sucessor de $\alpha-e_{2 n-1}+e_{2 n}$.

\footnotetext{
${ }^{2} \mathrm{Na}$ ordem lexicográfica, $\beta=\left(\beta_{1}, \ldots, \beta_{s}\right)$ sucede $\alpha=\left(\alpha_{1}, \ldots, \alpha_{s}\right)$ se, e somente se, $\alpha_{i}=\beta_{i}$ para $i=1,2, \ldots, l-1$ e $\beta_{l}>\alpha_{l}$ para algum $l \leq s$.
} 


\subsection{2 uma matriz especial}

Para cada $n \geq 1$, sejam $A \in M_{n \times n}(\mathbb{R})$ e $b \in \mathbb{R}$. Defina $T(A, 0, b)=A$ e, para $m \in \mathbb{Z}_{+}$, $m \geq 1$, defina

$$
T(A, m, b)=\left(\begin{array}{cccccc}
A & m b I_{n} & 0 & \ldots & 0 & 0 \\
-b I_{n} & A & (m-1) b I_{n} & \ldots & 0 & 0 \\
0 & -2 b I_{n} & A & \ldots & 0 & 0 \\
\vdots & \vdots & \vdots & \ddots & \vdots & \vdots \\
0 & 0 & 0 & \ldots & A & b I_{n} \\
0 & 0 & 0 & \ldots & -m b I_{n} & A
\end{array}\right)
$$

onde $I_{n} \in M_{n \times n}(\mathbb{R})$ denota a matriz identidade. Considere $T_{0}(m ; b)=T(0, m, b)$ com $0 \in$ $\mathbb{R}$. Dados $m_{1}, m_{2} \in \mathbb{Z}_{+}$e $b_{1}, b_{2} \in \mathbb{R}$ defina então $T_{0}\left(m_{1}, m_{2} ; b_{1}, b_{2}\right)=T\left(T_{0}\left(m_{1} ; b_{1}\right) ; m_{2} ; b_{2}\right)$ e indutivamente, defina

$$
T_{0}\left(m_{1}, \ldots, m_{r} ; b_{1}, \ldots, b_{r}\right)=T\left(T_{0}\left(m_{1}, \ldots m_{r-1} ; b_{1}, \ldots, b_{r-1}\right) ; m_{r} ; b_{r}\right) .
$$

O seguinte resultado será crucial para a demonstração da afirmação, e sua demonstração se encontra no Apêndice:

Teorema 6.6. Sejam $m \in \mathbb{Z}_{+} \backslash\{0\}$ e $b \in \mathbb{R}$. Então $\operatorname{Spec} T_{0}(m: b)=\{i b K ; K \in \mathcal{M}(m)\}$

Demonstração: No Apêndice.

Estamos interessados em estudar o espectro das matrizes (6.2.1). Antes disso, vamos obter uma expressão conveniente para o polinômio caracteristico de $T_{0}(m, b)$. Mais geralmente, vamos considerar o polinômio característico de uma matriz tridiagonal $A$ cuja diagonal principal é nula, isto é, se $A=\left(v_{i, j}\right)_{i, j=1, \ldots, m+1}$ temos $v_{i, j}=0$ se $|i-j| \neq 1$. Temos

$$
p(\lambda)=\operatorname{det}(\lambda-A)=\sum_{\sigma \in S_{m}}\left(\operatorname{sgn}(\sigma) \prod_{i=1}^{m+1}\left[\lambda \delta_{i, \sigma(i)}-v_{i, \sigma(i)}\right]\right) .
$$

Note que da forma como são distribuidos os zeros em $A$, segue que $v_{i, j}=0$ se $|i-j|>1$.

Se $\sigma$ não é formada (exclusivamente) por transposições disjuntas, então, existe $1 \leq$ $j \leq m+1$ tal que $|j-\sigma(j)|>1$. Então, no cálculo do polinômio característico, podemos supor que $\sigma=\left(\begin{array}{ll}j_{1} & j_{1}+1\end{array}\right) \ldots\left(\begin{array}{ll}j_{s} & j_{s}+1\end{array}\right)$, sendo $j_{1}, j_{1}+1, \ldots, j_{s}, j_{s}+1$ todos distintos. 
Assim, reescrevemos:

$$
\begin{aligned}
p(\lambda) & =\operatorname{det}(\lambda-A)=\sum_{\left(j_{1}, \ldots, j_{s}\right)}\left((-1)^{s} \prod_{i=1}^{m+1}\left[\lambda \delta_{i, \sigma(i)}-v_{i, \sigma(i)}\right]\right) \\
& =\sum_{\left(j_{1}, \ldots, j_{s}\right)}\left((-1)^{s} \prod_{i=1}^{s}\left[v_{j_{i}, j_{i}+1}\right]\left[v_{j_{i}+1, j_{i}}\right]\right) \lambda^{m+1-2 s},
\end{aligned}
$$

onde $\left(j_{1}, \ldots, j_{s}\right)$ satisfazem $j_{1}, j_{1}+1, \ldots, j_{s}, j_{s}+1$ todos disjuntos. Note que a expressão acima significa que todos os termos do polinômio característico tem a mesma paridade. Em particular, considere a matriz $\tilde{A}=\left(a_{i, j}\right)_{i, j}$, onde invertemos o sinal dos termos da diagonal abaixo da diagonal principal:

$$
a_{i, j}= \begin{cases}-v_{i, j} & \text { se } i-j=1 \\ v_{i, j} & \text { caso contrário. }\end{cases}
$$

Lema 6.7. Se $\lambda$ é autovalor de $A$, temos que $i \lambda$ é autovalor de $\tilde{A}$.

Demonstração: De fato, suponha que $\lambda$ seja autovalor de $A$, então,

$$
\begin{aligned}
\operatorname{det}(i \lambda-\tilde{A}) & =\sum_{\left(j_{1}, \ldots, j_{s}\right)}\left((-1)^{s} \prod_{i=1}^{s}\left[a_{j_{i}, j_{i}+1}\right]\left[a_{j_{i}+1, j_{i}}\right]\right)(i \lambda)^{m+1-2 s} \\
& =\sum_{\left(j_{1}, \ldots, j_{s}\right)}\left((-1)^{s} \prod_{i=1}^{s}\left[v_{j_{i}, j_{i}+1}\right]\left[-v_{j_{i}+1, j_{i}}\right]\right) i^{m+1} i^{-2 s} \lambda^{m+1-2 s} \\
& =i^{m+1} \sum_{\left(j_{1}, \ldots, j_{s}\right)}\left((-1)^{s} \prod_{i=1}^{s}\left[v_{j_{i}, j_{i}+1}\right]\left[v_{j_{i}+1, j_{i}}\right]\right)(-1)^{s}(-1)^{-s} \lambda^{m+1-2 s} \\
& =i^{m+1} \sum_{\left(j_{1}, \ldots, j_{s}\right)}\left((-1)^{s} \prod_{i=1}^{s}\left[v_{j_{i}, j_{i}+1}\right]\left[v_{j_{i}+1, j_{i}}\right]\right) \lambda^{m+1-2 s}=i^{m+1} \operatorname{det}(\lambda-A)=0 .
\end{aligned}
$$

A expressão (6.2.3), nos será útil na demonstração do lema a seguir.

Lema 6.8. Se, para quaisquer $m \in \mathbb{Z}_{+} \backslash\{0\}$ e $b \in \mathbb{R} \backslash\{0\}$ temos $\operatorname{Spec} T_{0}(m: b)=$ $\{i b K ; K \in \mathcal{M}(m)\}$, então $\operatorname{Spec} T_{0}\left(m_{1}, \ldots, m_{r} ; b_{1}, \ldots, b_{r}\right)=\left\{i \sum_{j=1}^{r} b_{j} K(j) ; K(j) \in\right.$ $\left.\mathcal{M}\left(m_{j}\right)\right\}$.

Demonstração: A demonstração é feita por indução em $r$, sendo o caso base verdadeiro pela hipótese do lema (na verdade o Teorema 6.6 vai nos garantir que a 
hipótese do lema é sempre verdadeira). Suponha, agora, que o resultado seja válido para $r$. Suponha ainda que $T_{0}\left(m_{1}, \ldots, m_{r} ; b_{1}, \ldots, b_{r}\right)$ seja diagonalizável. Neste caso, existe uma matriz $A$ tal que

$$
A T_{0}\left(m_{1}, \ldots, m_{r} ; b_{1}, \ldots, b_{r}\right) A^{-1}=D
$$

$\operatorname{com} D=\operatorname{diag}\left(\lambda_{1}, \ldots, \lambda_{l}\right)$ uma matriz diagonal. Considere a matriz $\tilde{A}=\operatorname{diag}(A, A, \ldots, A)$, com $m_{r+1}+1$ blocos na diagonal, e sua inversa $\tilde{A}^{-1}=\operatorname{diag}\left(A^{-1}, A^{-1}, \ldots, A^{-1}\right)$. Então,

$$
\tilde{A} T_{0}\left(m_{1}, \ldots, m_{r+1} ; b_{1}, \ldots, b_{r+1}\right) \tilde{A}^{-1}=T\left(D ; m_{r+1} ; b_{r+1}\right) .
$$

Vamos relembrar um resultado clássico da Algebra Linear³.

Denote por $M_{n}(K)$ o espaço das matrizes quadradas $n \times n$ com entradas em $K$. Defina o espaço $\mathfrak{M}_{n, k}=M_{n}\left(M_{k}(F)\right)$. Note que existe uma identificação canônica

$$
\mathfrak{M}_{n, k}=M_{n}\left(M_{k}(F)\right) \sim M_{k n}(F)
$$

sendo $F$ um corpo qualquer.

Podemos escrever $M_{n}\left(M_{k}(F)\right) \ni B=\left(B^{I, J}\right)_{I, J=1, \ldots, n}$, sendo $B^{I, J}$ a matrix $k \times k$ na $I$-ésima linha de $B$ e $J$-ésima coluna. Por outro lado, podemos escrever $M_{k n}(F) \ni B=$ $\left(b_{i, j}\right)_{i, j=1, \ldots, n k}$. Então, temos duas noções de determinante para $B$ :

$$
\operatorname{det}_{\mathfrak{M}_{n, k}}(B)=\sum_{\sigma \in S_{n}} \operatorname{sgn}(\sigma) B^{I, \sigma(I)}
$$

e

$$
\operatorname{det}_{M_{n k}(F)}(B)=\sum_{\tau \in S_{n k}} \operatorname{sgn}(\tau) b_{i, \tau(i)}
$$

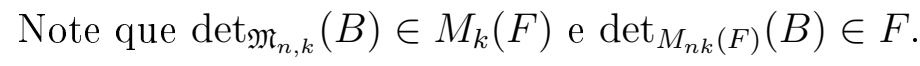

O resultado de algebra linear nos diz o seguinte: se as matrizes $B^{I, J}$ comutam entre si, então, vale que:

$$
\operatorname{det}_{M_{n k}(F)}(B)=\operatorname{det}_{M_{k}(F)}\left(\operatorname{det}_{\mathfrak{M}_{n, k}}(B)\right) .
$$

\footnotetext{
${ }^{3}$ Este resultado e sua demonstração se encontram em Kovacs [1999].
} 
Munido com este resultado, denote $D_{\lambda}=\lambda I_{l}-D=\operatorname{diag}\left(\lambda-\lambda_{1} \ldots, \lambda-\lambda_{l}\right)$. Sejam ainda $v_{i, j}$ os coeficientes da matriz $\left.T_{0}\left(m_{r+1} ; b_{r+1}\right)\right)$ e seja $p(\lambda)$ é o polinômio característico de $T_{0}\left(m_{r+1} ; b_{r+1}\right)$. Temos que

$$
\begin{aligned}
\operatorname{det}\left(\lambda-T\left(D ; m_{r+1} ; b_{r+1}\right)\right) & =\operatorname{det}\left(T\left(D_{\lambda} ; m_{r+1} ; b_{r+1}\right)\right) \\
& =\operatorname{det}_{M_{l}(\mathbb{C})}\left(\operatorname{det}_{\mathfrak{M}_{l, m_{r+1}}}\left(T\left(D_{\lambda} ; m_{r+1} ; b_{r+1}\right)\right)\right) \\
& =\operatorname{det}_{M_{l}(\mathbb{C})}\left(\operatorname{det}_{\mathfrak{M}_{l, m_{r+1}}}\left(\operatorname{diag}\left(D_{\lambda}, \ldots, D_{\lambda}\right)-T\left(0 \cdot I_{l} ; m_{r+1} ; b_{r+1}\right)\right)\right) \\
& =\operatorname{det}_{M_{l}(\mathbb{C})}\left(\sum_{\left(j_{1}, \ldots, j_{s}\right)}\left((-1)^{s} \prod_{i=1}^{s}\left[V^{j_{i}, j_{i}+1}\right]\left[V^{j_{i}+1, j_{i}}\right]\right) D_{\lambda}^{m_{r+1}+1-2 s}\right),
\end{aligned}
$$

onde $V^{i, j}$ uma matriz $l \times l$ que corresponde ao bloco na $i$-ésima linha e $j$-ésima coluna de $T\left(0 \cdot I_{l} ; m_{r+1} ; b_{r+1}\right)$ e $\left(j_{1} \ldots, j_{s}\right)$ satisfazem $j_{1}, j_{1}+1, \ldots, j_{s}, j_{s}+1$ são todos distintos.

Note que, se $i \neq j$ então $V^{i, j}=v_{i, j} I_{l}$. Além disso, $D_{\lambda}^{k}=\operatorname{diag}\left(\left(\lambda-\lambda_{1}\right)^{k}, \ldots,\left(\lambda-\lambda_{l}\right)^{k}\right)$. Daí, temos:

$$
\begin{aligned}
\operatorname{det}\left(\lambda-T\left(D ; m_{r+1} ; b_{r+1}\right)\right) & =\operatorname{det}_{M_{l}(\mathbb{C})}\left(\sum_{\left(j_{1}, \ldots, j_{s}\right)}\left((-1)^{s} \prod_{i=1}^{s}\left[v_{j_{i}, j_{i}+1}\right]\left[v_{j_{i}+1, j_{i}}\right]\right) D_{\lambda}^{m_{r+1}+1-2 s}\right) \\
& =\operatorname{det}_{M_{l}(\mathbb{C})}\left(\operatorname{diag}\left(p\left(\lambda-\lambda_{1}\right), \ldots, p\left(\lambda-\lambda_{l}\right)\right)\right) \\
& =\prod_{j=1}^{l} p\left(\lambda-\lambda_{l}\right) .
\end{aligned}
$$

Por hipótese do lema, $p(x)=0$ se, e somente se, $x \in\left\{i b_{r+1} K ; K \in \mathcal{M}\left(m_{r+1}\right)\right\}$. Assim, vale que

$$
\begin{aligned}
& \lambda \in \operatorname{Spec}_{0}\left(m_{1}, \ldots, m_{r+1} ; b_{1}, \ldots, b_{r+1}\right) \\
& \Uparrow \\
& \left(\lambda-\lambda_{l}\right) \in\left\{i b_{r+1} K ; K \in \mathcal{M}\left(m_{r+1}\right)\right\} \text { para algum } \lambda_{l} \in \operatorname{Spec}_{0}\left(m_{1}, \ldots, m_{r} ; b_{1}, \ldots, b_{r}\right) \\
& \mathbb{1} \\
& \lambda \in\left\{i \sum_{j=1}^{r+1} b_{j} K(j) ; K(j) \in \mathcal{M}\left(m_{j}\right)\right\}
\end{aligned}
$$

Resta concluirmos que $T_{0}\left(m_{1}, \ldots, m_{r+1} ; b_{1}, \ldots, b_{r+1}\right)$ é diagonalizável. Para isso, note que podemos escrever:

$$
\begin{aligned}
\tilde{A} T_{0}\left(m_{1}, \ldots, m_{r+1} ; b_{1}, \ldots, b_{r+1}\right) \tilde{A}^{-1} & =T\left(D ; m_{r+1} ; b_{r+1}\right) \\
& =\operatorname{diag}(D, \ldots, D)+T\left(0 \cdot I_{l} ; m_{r+1} ; b_{r+1}\right) .
\end{aligned}
$$


Como $T_{0}\left(m_{r+1} ; b_{r+1}\right)$ é diagonalizável (da hipótese do lema), segue que $T(0$. $\left.I_{l} ; m_{r+1} ; b_{r+1}\right)$ também é diagonalizável; assim, $T\left(D ; m_{r+1} ; b_{r+1}\right)$ é a soma de duas matriz diagonalizáveis que comutam ${ }^{4}$ e, portanto, elas podem ser simultaneamente diagonalizadas; logo a soma é diagonalizável.

Vamos, agora, provar o Lema 6.2.

Lema 6.9. Nas condições e notações do Lema 6.1 Existe uma ordenação de $\Lambda_{s}^{m}$ tal que

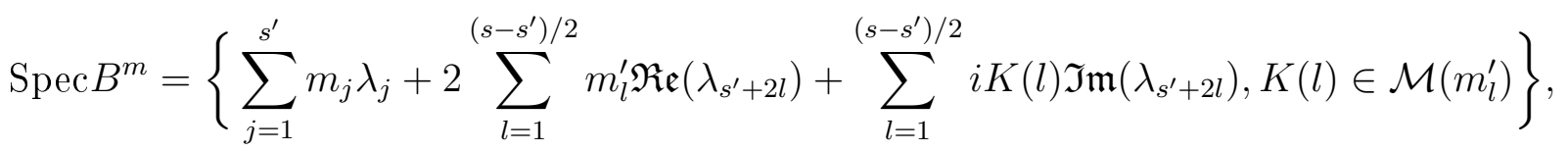
sendo $m_{1}+\ldots m_{s}^{\prime}+m_{1}^{\prime}+m_{\left(s-s^{\prime}\right) / 2}^{\prime}=m$.

Demonstração: Relembrando, temos que, para cada $m$, a matriz $B^{m}$ acima surge quando consideramos o sistema de equações

$$
\sum_{j=1}^{s} \sum_{k=1}^{s} \alpha_{k} \partial^{e_{k}} a_{j}(0) \partial^{\alpha+e_{j}-e_{k}} u(0)+c(0) \partial^{\alpha} u(0)=\partial^{\alpha} f(0)+R^{\alpha}, \quad \alpha \in \Lambda_{s}^{m}
$$

Podemos supor, sem perda de generalidade, que $D L(0)=\left(\partial^{e_{i}} a_{j}(0)\right)_{i, j=1, \ldots, s}$ esteja em sua forma canônica de Jordan. Mais ainda, podemos supor que, sendo $\lambda_{s^{\prime}+1}^{\prime}, \ldots, \lambda_{s}$ os autovalores complexos de $D L(0)$, então $\lambda_{s^{\prime}+2 j}$ e $\lambda_{s^{\prime}+2 j-1}$ sejam autovalores conjugados. Para facilitar a notação, defina $2 r=s-s^{\prime}$ (note que $r$ é inteiro, pois os autovalores complexos sempre aparecem aos pares) e denote $a_{j}=\mathfrak{R e}\left(\lambda_{s^{\prime}+2 j-1}\right)$ e $b_{j}=\operatorname{Im}\left(\lambda_{s^{\prime}+2 j-1}\right)$. Queremos reescrever (6.2.4) de maneira mais conveniente. Para isso, observe que, restringindo $D L(0)=\left(\partial^{e_{k}} a_{j}(0)\right)_{k, j}$ à $1 \leq j, k \leq s^{\prime}$, obtemos uma matriz do tipo

$$
\left(\begin{array}{ccccccc}
\lambda_{1} & \mu_{1} & 0 & 0 & \ldots & 0 & 0 \\
0 & \lambda_{2} & \mu_{2} & 0 & \ldots & 0 & 0 \\
0 & 0 & \lambda_{3} & \mu_{3} & \ldots & 0 & 0 \\
\vdots & \vdots & \ddots & \ddots & \ddots & \vdots & \vdots \\
0 & 0 & \ldots & \ldots & 0 & \lambda_{s^{\prime}-1} & \mu_{s^{\prime}-1} \\
0 & 0 & \ldots & \ldots & 0 & 0 & \lambda_{s^{\prime}}
\end{array}\right)
$$

\footnotetext{
${ }^{4}$ Este resultado se encontra em "GOLAN, J.S. The Linear Algebra a Beginning Graduate Student Ought to Know. 2nd Edition, Springer"( Proposição 12.18)
} 
onde $\mu_{j} \in\{0,1\}$. Assim:

$$
\sum_{j=1}^{s^{\prime}} \sum_{k=1}^{s^{\prime}} \alpha_{k} \partial^{e_{k}} a_{j}(0) \partial^{\alpha+e_{j}-e_{k}} u(0)=\sum_{j=1}^{s^{\prime}} \alpha_{j} \lambda_{j} \partial^{\alpha} u(0)+\sum_{j=1}^{s^{\prime}-1} \mu_{j} \alpha_{j+1} \partial^{\alpha+e_{j}-e_{j+1}} u(0) .
$$

Analogamente, restringindo $D L(0)=\left(\partial^{e_{k}} a_{j}(0)\right)_{k, j}$ à $s^{\prime}+1 \leq j, k \leq s$, obtemos uma matriz do tipo

$$
\left(\begin{array}{ccccccc}
J_{1} & \epsilon_{1} I_{2} & 0 & 0 & \ldots & 0 & 0 \\
0 & J_{2} & \epsilon_{2} I_{2} & 0 & \ldots & 0 & 0 \\
0 & 0 & J_{3} & \epsilon_{3} I_{2} & \ldots & 0 & 0 \\
\vdots & \vdots & \ddots & \ddots & \ddots & \vdots & \vdots \\
0 & 0 & \ldots & \ldots & 0 & J_{r-1} & \epsilon_{r-1} I_{2} \\
0 & 0 & \ldots & \ldots & 0 & 0 & J_{r}
\end{array}\right)
$$

sendo $J_{l}=\left(\begin{array}{cc}a_{l} & b_{l} \\ -b_{l} & a_{l}\end{array}\right)$ e $\epsilon_{j} \in\{0,1\}, j=1, \ldots, r-1$. Para facilitar a notação, vamos estabelecer as seguintes convenções:

- $\tilde{\alpha}_{k}=\alpha_{s^{\prime}+k}$

- $\tilde{e}_{j}=e_{s^{\prime}+j}$.

Daí,

$$
\begin{aligned}
\sum_{j=s^{\prime}+1}^{s} & \sum_{k=s^{\prime}+1}^{s} \alpha_{k} \partial^{e_{k}} a_{j}(0) \partial^{\alpha+e_{j}-e_{k}} u(0)=\sum_{j=1}^{2 r} \sum_{k=1}^{2 r} \tilde{\alpha}_{k} \partial^{e_{s^{\prime}+k}} a_{s^{\prime}+j}(0) \partial^{\alpha+\tilde{e}_{j}-\tilde{e}_{k}} u(0) \\
= & \sum_{j=1}^{r}\left(\tilde{\alpha}_{2 j-1}+\tilde{\alpha}_{2 j}\right) a_{j} \partial^{\alpha} u(0)+ \\
& +\sum_{j=1}^{r} \tilde{\alpha}_{2 j} b_{j} \partial^{\alpha+\tilde{e}_{2 j-1}-\tilde{e}_{2 j}} u(0)-\sum_{j=1}^{r} \tilde{\alpha}_{2 j-1} b_{j} \partial^{\alpha+\tilde{e}_{2 j}-\tilde{e}_{2 j-1}} u(0)+ \\
& +\sum_{j=1}^{r-1} \epsilon_{j}\left(\tilde{\alpha}_{2 j+1} \partial^{\alpha+\tilde{e}_{2 j-1}-\tilde{e}_{2 j+1}} u(0)+\tilde{\alpha}_{2 j+2} \partial^{\left.\alpha+\tilde{e}_{2 j}-\tilde{e}_{2 j+2} u(0)\right) .}\right.
\end{aligned}
$$


Assim, podemos reescrever (6.2.4) da seguinte forma:

$$
\begin{aligned}
\sum_{j=1}^{s^{\prime}} \alpha_{j} \lambda_{j} \partial^{\alpha} u(0) & +\sum_{j=1}^{r}\left(\tilde{\alpha}_{2 j-1}+\tilde{\alpha}_{2 j}\right) a_{j} \partial^{\alpha} u(0)+ \\
& +\sum_{j=1}^{r} b_{j} B_{j}^{\alpha}+\sum_{j=1}^{s^{\prime}-1} \mu_{j} M_{j}^{\alpha}+\sum_{j=1}^{r-1} \epsilon_{j} E_{j}^{\alpha}+c(0) \partial^{\alpha} u(0)=\partial^{\alpha} f(0)+R^{\alpha}
\end{aligned}
$$

com $\mu_{j}$ e $\epsilon_{j}$ valendo 0 ou 1 e sendo

$$
\begin{aligned}
M_{j}^{\alpha} & =\alpha_{j+1} \partial^{\alpha+e_{j}-e_{j+1}} u(0) \\
B_{j}^{\alpha} & =\tilde{\alpha}_{2 j} \partial^{\alpha+\tilde{e}_{2 j-1}-\tilde{e}_{2 j}} u(0)-\tilde{\alpha}_{2 j-1} \partial^{\alpha+\tilde{e}_{2 j}-\tilde{e}_{2 j-1}} u(0) \\
E_{j}^{\alpha} & =\tilde{\alpha}_{2 j+1} \partial^{\alpha+\tilde{e}_{2 j-1}-\tilde{e}_{2 j+1}} u(0)+\tilde{\alpha}_{2 j+2} \partial^{\alpha+\tilde{e}_{2 j}-\tilde{e}_{2 j+2}} u(0) .
\end{aligned}
$$

Queremos, agora, estabelecer em $\Lambda_{s}^{m}$ uma ordem de modo que o determinante de $B^{m}$ possa ser calculado por blocos e, em cada bloco, as constantes $\mu_{j}$ e $\epsilon_{j}$ sejam nulas.

Note que:

$$
\Lambda_{s}^{m}=\bigcup_{m_{0}+m_{1}+\cdots+m_{r}=m} \Lambda_{s^{\prime}}^{m_{0}} \times \Lambda_{2}^{m_{1}} \times \cdots \times \Lambda_{2}^{m_{r}}
$$

Vamos, agora, ordenar esta união colocando a ordem lexicográfica nos índices, isto é:

$$
\left\{\Lambda_{s^{\prime}}^{m_{0}} \times \Lambda_{2}^{m_{1}} \times \cdots \times \Lambda_{2}^{m_{r}} ; m_{0}+m_{1}+\cdots+m_{r}=m\right\}
$$

sucede

$$
\left\{\Lambda_{s^{\prime}}^{k_{0}} \times \Lambda_{2}^{k_{1}} \times \cdots \times \Lambda_{2}^{k_{r}} ; k_{0}+k_{1}+\cdots+k_{r}=m\right\}
$$

se, e somente se, $m_{j}=k_{j}$ para $0 \leq j \leq l$ e $m_{l+1}>k_{l+1}$ para algum $l$. Em particular, se $m_{j+1} \neq 0$, então

$$
\Lambda_{s^{\prime}}^{m_{0}} \times \Lambda_{2}^{m_{1}} \times \ldots \Lambda_{2}^{m_{j}+1} \times \Lambda_{2}^{m_{j+1}-1} \times \Lambda_{2}^{m_{r}}
$$

sucede

$$
\Lambda_{s^{\prime}}^{m_{0}} \times \Lambda_{2}^{m_{1}} \times \ldots \Lambda_{2}^{m_{j}} \times \Lambda_{2}^{m_{j+1}} \times \Lambda_{2}^{m_{r}} .
$$


Agora, dote $\Lambda_{s^{\prime}}^{m_{0}} \times \Lambda_{2}^{m_{1}} \times \cdots \times \Lambda_{2}^{m_{r}}$ com a ordem produto, e cada parcela com a ordem lexicográfica. Com está ordem temos:

Fato 1 A matriz $B^{m}$ é triangular por blocos. Além disso, cada bloco da diagonal está em correspondência bionívoca com os conjuntos da forma $\left\{\Lambda_{s^{\prime}}^{m_{0}} \times \Lambda_{2}^{m_{1}} \times \cdots \times \Lambda_{2}^{m_{r}} ; m_{0}+\right.$ $\left.m_{1}+\cdots+m_{r}=m\right\}$. Em particular, no cálculo do espectro de $B^{m}$ podemos considerar $\epsilon_{j}=0$.

\section{De fato:}

Vamos denotar por $B_{\alpha, \beta}$ o elemento de $B^{m}$ na $\alpha$-ésima linha e $\beta$-ésima coluna. Temos que $B_{\alpha, \beta}$ é o termo que multiplica $\partial^{\beta} u(0)$ na equação (6.2.4). Seja $\alpha \in A_{m_{0}, \ldots, m_{r}}:=$ $\Lambda_{s^{\prime}}^{m_{0}} \times \Lambda_{2}^{m_{1}} \times \cdots \times \Lambda_{2}^{m_{r}}$. Vamos mostrar que se $\beta \in A_{k_{0}, \ldots, k_{r}}:=\Lambda_{s^{\prime}}^{k_{0}} \times \Lambda_{2}^{k_{1}} \times \cdots \times \Lambda_{2}^{k_{r}} \mathrm{e}$ $A_{m_{0}, \ldots, m_{r}}$ sucede $A_{k_{0}, \ldots, k_{r}}$, então $B_{\alpha, \beta}=0$.

Diremos que um termo $a \partial^{\nu} u(0)$ pertence ao bloco $A_{k_{0}, \ldots, k_{r}}$ se $\nu \in A_{k_{0}, \ldots, k_{r}}$.

Note que se $\alpha_{j+1} \neq 0$, para $j=1, \ldots, s^{\prime}-1$, então $\alpha+e_{j}-e_{j+1} \in A_{m_{0}, \ldots, m_{r}}$; isto é, os termos $M_{j}^{\alpha}$ em (6.2.5) pertencem ao bloco $A_{m_{0}, \ldots, m_{r}}$.

Note ainda que se $\tilde{\alpha}_{2 j} \neq 0, j=1, \ldots, r$, então $\alpha+\tilde{e}_{2 j-1}-\tilde{e}_{2 j} \in A_{m_{0}, \ldots, m_{r}}$; analogamente, se $\tilde{\alpha}_{2 j-1} \neq 0$, então $\alpha+\tilde{e}_{2 j}-\tilde{e}_{2 j-1} \in A_{m_{0}, \ldots, m_{r}}$. De modo que os termos em $B_{j}^{\alpha}$ pertencem a $A_{m_{0}, \ldots, m_{r}}$.

Finalmente, note que se $\tilde{\alpha}_{2 j+1} \neq 0, j=1, \ldots, r-1$, então $\alpha+\tilde{e}_{2 j-1}-\tilde{e}_{2 j+1} \in$ $A_{m_{0}, \ldots, m_{j}+1, m_{j}-1, \ldots, m_{r}}$ que sucede $A_{m_{0}, \ldots, m_{r}}$. Analogamente, se $\tilde{\alpha}_{2 j+2} \neq 0$, então $\alpha+\tilde{e}_{2 j}-\tilde{e}_{2 j+2} \in A_{m_{0}, \ldots, m_{j}+1, m_{j}-1, \ldots, m_{r}}$ que sucede $A_{m_{0}, \ldots, m_{r}}$. Portanto, os termos em $E_{j}^{\alpha}$ não pertence a $A_{k_{0}, \ldots, k_{r}}$.

A correspondencia biunívoca entre os blocos da diagonal e os conjuntos

$$
\left\{\Lambda_{s^{\prime}}^{m_{0}} \times \Lambda_{2}^{m_{1}} \times \cdots \times \Lambda_{2}^{m_{r}} ; m_{0}+m_{1}+\cdots+m_{r}=m\right\}
$$

é imediata.

Para verificar que no cálculo dos autovalores de $B^{m}$ podemos considerar $\epsilon_{j}=0$ basta observar que se $\alpha \in A_{m_{0}, \ldots, m_{r}}$ então os termos em $E_{j}^{\alpha}$ não pertencem a $A_{m_{0}, \ldots, m_{r}}$; isto é, eles não interferem nos blocos da diagonal.

Fato 2: Seja $B^{\prime}$ um bloco da diagonal de $B^{m}$ associado à $A_{m_{0}, \ldots, m_{r}}$. Então, $B^{\prime}$ é triangular 
superior em blocos e, para o cálculo dos autovalores de $B$, podemos considerar que $\mu_{j}=0$.

\section{De fato:}

Seja $\alpha \in A_{m_{0}, \ldots, m_{r}}$ e $\alpha_{j+1}, j=1, \ldots, s^{\prime}-1$. Então, $\alpha+e_{j}-e_{j+1}$ sucede $\alpha$. Daí, $B^{\prime}$ é triangular por blocos.

Escrevendo $\alpha=\left(\alpha_{0}, \tilde{\alpha}\right) \in A_{m_{0}, \ldots, m_{r}}, \alpha_{0} \in \Lambda_{s^{\prime}}^{m_{0}}$ estamos associando cada bloco da diagonal a um conjunto da forma $\left\{\alpha_{0}\right\} \times \Lambda_{2}^{m^{1}} \times \cdots \times \Lambda_{2}^{m_{r}}$.

Podemos, então, considerar $\mu_{j}=0$ no cálculo dos autovalores de $B^{\prime}$.

Fato 3: Seja $B$ bloco diagonal da matriz $B^{\prime}$ acima. Então, $B$ é da forma

$$
\lambda I+T_{0}\left(m_{1}, \ldots, m_{r} ; b_{1}, \ldots, b_{r}\right)
$$

sendo

$$
\lambda=\sum_{j=1}^{s^{\prime}} \alpha_{j} \lambda_{j}+\sum_{j=1}^{r}\left(\tilde{\alpha}_{2 j-1}-\tilde{\alpha}_{2 j}\right) a_{j} .
$$

\section{De fato:}

Escrevendo $\alpha=\left(\alpha_{0}, \tilde{\alpha}\right)$ como acima, os passos anteriores nos dizem que o bloco $B^{\prime}$ é obtido a partir das equações (6.2.5) quando consideramos $\alpha_{0}$ fixado, $\mu_{j}=\epsilon_{k}=0 \mathrm{e}$ $\tilde{\alpha} \in \Lambda_{2}^{m^{1}} \times \cdots \times \Lambda_{2}^{m_{r}}$.

Observe que se $m_{j} \neq 0, j=1, \ldots, r$, então, para $\alpha_{0}, \tilde{\alpha}_{1}, \tilde{\alpha}_{2}, \ldots, \tilde{\alpha}_{2 j-3}, \tilde{\alpha}_{2 j-2}$ fixados, temos

$$
B_{\alpha, \alpha+\tilde{e}_{2 j-1}-\tilde{e}_{2 j}}=\tilde{\alpha}_{2 j}
$$

se $\tilde{\alpha}_{2 j} \neq 0$, e

$$
B_{\alpha, \alpha+\tilde{e}_{2 j}-\tilde{d e}_{2 j-1}}=-\tilde{\alpha}_{2 j-1}
$$

se $\tilde{\alpha}_{2 j-1} \neq 0$. Lembre-se que a notação $B_{\alpha, \beta}$ indica um elemento na matriz $B^{m}$. Agora, observando que vale [P1] e [P1'] segue o Fato 3. 
Finalmente, segue do Teorema 6.6 que a hipótese do Lema 6.8 é satisfeita. Portanto, segue o resultado. 
Apêndice

$\mathcal{A}$

\section{Geodésicas}

Este apêndice segue de perto o livro de geometria Riemanniana de Manfredo do Carmo [Carmo, 1988]. Entre parênteses, nas proposições apresentadas, colocamos o capítulo e o número da proposição no livro de Manfredo, por exemplo:

Proposição A.? (Cap. I - Prop 1.3)

significa que a proposição enunciada é na verdade a proposição 1.3 do Capítulo I no livro do Manfredo.

Definição A.1. Seja $M$ uma variedade, então uma Métrica Riemanniana em $M$ é um produto interno $g_{p}: T_{p} M \times T_{p} M \rightarrow \mathbb{R}$ definido em cada espaço tangente de $M$, de modo que, para qualquer sistema de coordenadas $x_{1}, \ldots, x_{n}$, e para quaisquer $i, j$, a aplicação abaixo é suave:

$$
M \ni p \mapsto g_{p}\left(\frac{\partial}{\partial x_{i}}(p), \frac{\partial}{\partial x_{j}}(p)\right)
$$

A dupla $(M, g)$ é chamada de variedade Riemanniana.

Sejam $\left(M_{1}, g_{1}\right)$ e $\left(M_{2}, g_{2}\right)$ variedades Riemannianas, então, é possivel definir, de maneira natural, uma métrica Riemanniana em $M_{1} \times M_{2}$. Considere $\pi_{j}: M_{1} \times M_{2} \rightarrow M_{j}$ a projeção usual e defina em $M_{1} \times M_{2}$ a seguinte métrica:

$$
\left(g_{1} \times g_{2}\right)_{(p, q)}(u, v)=\left(g_{1}\right)_{p}\left(d \pi_{1} \cdot u, d \pi_{1} \cdot v\right)+\left(g_{2}\right)_{q}\left(d \pi_{2} \cdot u, d \pi_{2} \cdot v\right)
$$

É facil ver que $g_{1} \times g_{2}$ de fato é uma métrica Riemanniana. Observe que, na métrica produto, se $u \in T_{(p, q)} M_{1}$ e $v \in T_{(p, q)} M_{2}$ então $g_{1} \times g_{2}(u, v)=0$. Em particular, se $u, v \in T_{(p, q)} M_{j}$ então $g_{1} \times g_{2}(u, v)=g_{j}(u, v)$. 
Quando consideramos $\mathbb{S}^{1}$ com a métrica induzida por $\mathbb{R}^{2}$ e o toro $\mathbb{T}^{n}=\mathbb{S}^{1} \times \cdots \times \mathbb{S}^{1}$ com a métrica produto, dizemos que este é o toro plano.

Definição A.2. Denotemos por $\mathfrak{X}^{\infty}(M)$ o espaço dos campos de vetores suaves de $M$. Uma conexão afim em uma variedade diferenciável $M$ é uma aplicação bilinear

$$
\nabla: \mathfrak{X}^{\infty}(M) \times \mathfrak{X}^{\infty}(M) \rightarrow \mathfrak{X}^{\infty}(M) \quad, \quad \nabla(X, Y)=\nabla_{X} Y
$$

tal que, para quaisquer $X, Y, Z \in \mathfrak{X}^{\infty}(M)$ e $f, g \in C^{\infty}(M), \nabla$ satisfaz as seguintes propriedades

- $\nabla_{f X} Z=f \nabla_{X} Z$

- $\nabla_{X}(f Y)=f \nabla_{X} Y+X(f) Y$.

Fixando um sistema de coordenadas locais e escrevendo

$$
X=\sum_{i} a_{i}(x) X_{i} \quad \text { e } \quad Y=\sum_{j} b_{j}(x) X_{j}
$$

onde $X_{i}=\frac{\partial}{\partial x_{i}}$, temos

$$
\begin{aligned}
\nabla_{X} Y & =\nabla_{\sum_{i} a_{i}(x) X_{i}}\left(\sum_{j} b_{j}(x) X_{j}\right)=\sum_{i} a_{i}(x) \nabla_{X_{i}}\left(\sum_{j} b_{j}(x) X_{j}\right) \\
& =\sum_{i} \sum_{j} a_{i}(x)\left(b_{j}(x) \nabla_{X_{i}} X_{j}+X_{i}\left(b_{j}(x)\right) X_{j}\right)
\end{aligned}
$$

daí, escrevendo $\nabla_{X_{i}} X_{j}=\sum_{k} \Gamma_{i j}^{k} X_{k}$, obtemos a seguinte fórmula explicita para a conexão:

$$
\nabla_{X} Y=\sum_{k}\left(\sum_{i, j} a_{i}(x) b_{j}(x) \Gamma_{i j}^{k}+X\left(b_{k}(x)\right)\right) X_{k}
$$

Ou seja, $\nabla_{X} Y(p)$ depende apenas de $a_{i}(p), b_{k}(p)$ e das derivadas $X\left(b_{k}\right)(p)$. Em particular, podemos considerar $X$ e $Y$ campos ao longo de uma curva. $\Gamma_{i j}^{k}$ são chamados de Símbolos de Christoffel e, claramente, eles determinam a conexão afim.

Proposição A.3 (Cap. II - Prop. 2.2). Seja $M$ uma variedade Riemanniana com uma conexão afim $\nabla$. Existe uma única correspondencia entre um campo vetorial $V$ ao longo de uma curva suave $c: I \rightarrow M$ e um outro campo vetorial, chamado derivada covariante, $\frac{D V}{d t}$ ao longo de $c$ tal que:

- $\frac{D}{d t}(V+W)=\frac{D V}{d t}+\frac{D W}{d t}$ 
- $\frac{D}{d t}(f V)=\frac{d f}{d t} V+f \frac{D V}{d t}$, onde $f: I \rightarrow \mathbb{R}$ é uma função suave

- Se $V$ é induzido por um campo $Y \in \mathfrak{X}^{\infty}(M)$, isto é, se $V(t)=Y(c(t))$, então $\frac{D V}{d t}=\nabla_{\dot{c}} Y$, onde $\dot{c}=\frac{d c}{d t}$

Definição A.4. Seja $M$ uma variedade Riemanniana com conexão afim $\nabla$. Um campo $V$ ao longo de $c: I \rightarrow M$ é chamado de paralelo quando $\frac{D V}{d t}=0$

Proposição A.5 (Cap. II - Prop. 2.6). Sejam $M$ uma variedade Riemanniana com conexão afim $\nabla, c: I \rightarrow M$ uma curva suave em $M$ e $V_{0} \in T_{c\left(t_{0}\right)} M$. Então, existe um único campo de vetores paralelo $V$ ao longo de $c$, tal que $V\left(t_{0}\right)=V_{0}$. $V(t)$ é chamado o transporte paralelo de $V_{0}$

Definição A.6. Uma conexão afim $\nabla$ em uma variedade Riemanniana $(M, g)$ é dita compatível com a métrica $g$ quando, quaisquer dois campos de vetores ao longo de uma curva $c$ satisfazem:

$$
\frac{d}{d t} g(V, W)=g\left(\frac{D V}{d t}, W\right)+g\left(V, \frac{D W}{d t}\right)
$$

ou, equivalentemente

$$
X\langle Y, Z\rangle=\left\langle\nabla_{X} Y, Z\right\rangle+\left\langle Y, \nabla_{X} Z\right\rangle, \forall X, Y, Z \in \mathfrak{X}^{\infty}(M)
$$

A conexão $\nabla$ é dita simétrica quando $\nabla_{X} Y-\nabla_{Y} X=[X, Y]$, para todo $X, Y \in$ $\mathfrak{X}^{\infty}(M)$.

Teorema A.7 (Teorema de Levi-Civita). Dada uma variedade Riemanniana $(M,\langle\cdot, \cdot\rangle)$, existe uma única conexão afim $\nabla$ que é simétrica e compativel com a métrica. Esta é chamada de conexão de Levi-Civita ou simplesmente a conexão da variedade Riemanniana $M$

Demonstração: Faremos aqui apenas um esboço da demonstração. Os detalhes se resumem a verificar manualmente que as identidades são satisfeitas.

Suponha a existência desta conexão $\nabla$, então, dados campos $X, Y, Z \in \mathfrak{X}^{\infty}(M)$ segue da compatibilidade com a métrica que

$$
\left\{\begin{array}{l}
X\langle Y, Z\rangle=\left\langle\nabla_{X} Y, Z\right\rangle+\left\langle Y, \nabla_{X} Z\right\rangle \\
Y\langle Z, X\rangle=\left\langle\nabla_{Y} Z, X\right\rangle+\left\langle Z, \nabla_{Y} X\right\rangle \\
Z\langle X, Y\rangle=\left\langle\nabla_{Z} X, Y\right\rangle+\left\langle X, \nabla_{Z} Y\right\rangle
\end{array}\right.
$$


daí, usando a simetria temos

$$
\begin{aligned}
X\langle Y, Z\rangle & +Y\langle Z, X\rangle-Z\langle X, Y\rangle \\
& =\langle[X, Z], Y\rangle+\langle[Y, Z], X\rangle+\langle[X, Y], Z\rangle+2\left\langle Z, \nabla_{Y} X\right\rangle
\end{aligned}
$$

e, portanto, se $\nabla$ existe segundo as condições do teorema ela satisfaz a expressão acima; portanto, é única. Definindo $\nabla$ pela expressão acima, verifica-se que $\nabla$ de fato é uma conexão e satisfaz as propriedades desejadas.

A expressão que obtivemos na demonstração anterior é útil quando queremos calcular os símbolos de Christoffel da conexão de Levi-Civita em coordenadas locais. De fato, sejam $X_{i}=\partial_{x_{i}}$ em um determinado sistema de coordenadas locais fixado. Então:

$$
\left\langle\nabla_{X_{i}} X_{j}, X_{l}\right\rangle=\left\langle\sum_{k} \Gamma_{i j}^{k} X_{k}, X_{l}=\sum_{k} \Gamma_{i j}^{k} g_{k l}\right.
$$

onde $g_{k l}=\left\langle X_{k}, X_{l}\right\rangle$. Além disso, $\left[X_{i}, X_{j}\right]=0$ para quaisquer $i, j$ e, portanto, segue de (A.0.1) que

$$
\begin{aligned}
2 \sum_{k} \Gamma_{i j}^{k} g_{k l} & =X_{j}\left\langle X_{i}, X_{l}\right\rangle+X_{i}\left\langle X_{l}, X_{j}\right\rangle-X_{l}\left\langle X_{j}, X_{i}\right\rangle \\
& =\frac{\partial}{\partial x_{j}} g_{i l}+\frac{\partial}{\partial x_{i}} g_{l j}-\frac{\partial}{\partial x_{l}} g_{i j}
\end{aligned}
$$

como $\left(g_{k l}\right)_{k, l}$ é uma matriz invertível ${ }^{1}$, com inversa $\left(g^{k l}\right)_{k, l}$, temos que

$$
\Gamma_{i j}^{k}=\frac{1}{2} \sum_{l}\left(\frac{\partial}{\partial x_{j}} g_{i l}+\frac{\partial}{\partial x_{i}} g_{l j}-\frac{\partial}{\partial x_{l}} g_{i j}\right) g^{l k}
$$

Observação A.8. Seja $M$ a circunferência de raio $r$ e centrada na origem em $\mathbb{R}^{2}$, com a métrica Riemanniana induzida pelo produto interno usual em $\mathbb{R}^{2}$. Considere a carta local $I \ni \theta \mapsto(r \cos \theta, r \sin \theta) \in M \subseteq \mathbb{R}^{2}$, para um intervalo apropriado. Temos que $\partial_{\theta}=\frac{\partial}{\partial \theta}=(-r \sin \theta, r \cos \theta)$ e $\left\langle\partial_{\theta}, \partial_{\theta}\right\rangle=r^{2}$. Como $M$ é unidimensional, existe um único simbolo de Christoffel, dado por:

$$
\nabla_{\partial_{\theta}} \partial_{\theta}=\Gamma_{\theta \theta}^{\theta} \partial_{\theta}=\frac{1}{2}\left(\frac{\partial}{\partial \theta}\left\langle\partial_{\theta}, \partial_{\theta}\right\rangle+\frac{\partial}{\partial \theta}\left\langle\partial_{\theta}, \partial_{\theta}\right\rangle-\frac{\partial}{\partial \theta}\left\langle\partial_{\theta}, \partial_{\theta}\right\rangle\right)\left\langle\partial_{\theta}, \partial_{\theta}\right\rangle \partial_{\theta}=0
$$

\footnotetext{
${ }^{1}\left(g_{k l}\right)_{k, l}$ define um produto interno e, portanto, é uma matriz positiva definida, ou seja, todos os autovalores são reais positivos. Em particular, ela não possui autovalor nulo, e assim tem Kernel trivial.
} 
Assim, dados dois campos $X=f(\theta) \partial_{\theta}$ e $Y=g(\theta) \partial_{\theta}$ temos que

$$
\nabla_{X} Y=f \nabla_{\partial_{\theta}}\left(g \partial_{\theta}\right)=f g \nabla_{\partial_{\theta}} \partial_{\theta}+f \partial_{\theta}(g) \partial_{\theta}=f(\theta) g^{\prime}(\theta) \partial_{\theta}
$$

Proposição A.9. Sejam $M_{j}, j=1, \ldots, m$, variedades riemannianas com conexões de Levi-Civita $\nabla^{j}$. Então, a conexão de Levi-Civita de $M_{1} \times \cdots \times M_{n}$ satisfaz

$$
\nabla_{X^{1}+\cdots+X^{n}}\left(Y^{1}+\ldots Y^{n}\right)=\sum_{j}^{n} \nabla_{X^{j}}^{j} Y^{j}, X^{j}, Y^{j} \in(X)^{\infty}\left(M_{j}\right)
$$

Demonstração: Basta provar o resultado para $n=2$. Sabemos que, localmente, um campo em $M_{1} \times M_{2}$ é localmente da forma

$$
Z=\sum_{l} f_{l} X_{l}+\sum_{t} g_{t} Y_{t}
$$

onde $X_{j}=\partial_{x_{j}}, Y_{k}=\partial_{y_{k}}$ e $f_{j}^{1}, f_{k}^{2}$ são funções suaves definidas em $M_{1} \times M_{2}$. Note que, quando restringimos $\nabla$ a campos tangentes a $M_{j}$ temos que $\nabla$ define uma conexão em $M_{j}$ e, portanto, da unicidade da conexão de Levi-Civita, segue que

$$
\left\{\begin{array}{l}
\nabla_{X_{j}} X_{k}=\nabla_{X_{j}}^{1} X_{k} \\
\nabla_{Y_{j}} Y_{k}=\nabla_{X_{j}}^{2} X_{k}
\end{array}\right.
$$

logo, $\nabla_{X_{j}} X_{k}$ é tangente à $M_{1}$ e $\nabla_{Y_{j}} Y_{k}$ é tangente à $M_{2}$. Vamos denotar por $\langle\cdot, \cdot\rangle$ a métrica em $M_{1} \times M_{2}$ e por $\langle\cdot, \cdot\rangle_{j}$ a métrica em $M_{j}$. Como a conexão é compatível com a métrica, temos:

$$
\left\langle\nabla_{X_{j}} Y_{k}, Z\right\rangle=X_{j}\left\langle Y_{k}, Z\right\rangle-\left\langle Y_{k}, \nabla_{X_{j}} Z\right\rangle
$$

mas $\langle\cdot, \cdot\rangle$ é a métrica produto, $\log 0\left\langle Y_{k}, X_{j}\right\rangle=0$ e assim

$$
\begin{aligned}
\left\langle\nabla_{X_{j}} Y_{k}, Z\right\rangle & =X_{j}\left\langle Y_{k}, \sum_{l} f_{l} X_{l}+\sum_{t} g_{t} Y_{t}\right\rangle-\left\langle Y_{k}, \nabla_{X_{j}}\left(\sum_{l} f_{l} X_{l}+\sum_{t} g_{t} Y_{t}\right)\right\rangle \\
& =\sum_{t} X_{j}\left\langle Y_{k}, g_{t} Y_{t}\right\rangle_{2}-\sum_{l}\left\langle Y_{k}, \nabla_{X_{j}}\left(f_{l} X_{l}\right)\right\rangle-\sum_{t}\left\langle Y_{k}, \nabla_{X_{j}}\left(g_{t} Y_{t}\right)\right\rangle
\end{aligned}
$$


mas $\nabla_{X_{j}}\left(f_{l} X_{l}\right)$ é tangente à $M_{1}$, portanto, $\left\langle Y_{k}, \nabla_{X_{j}}\left(f_{l} X_{l}\right)\right\rangle=0$. Assim,

$$
\begin{aligned}
\left\langle\nabla_{X_{j}} Y_{k}, Z\right\rangle & =\sum_{t} X_{j}\left\langle Y_{k}, g_{t} Y_{t}\right\rangle_{2}-\sum_{t}\left\langle Y_{k}, \nabla_{X_{j}}\left(g_{t} Y_{t}\right)\right\rangle \\
& =\sum_{t}\left(X_{j}\left\langle Y_{k}, g_{t} Y_{t}\right\rangle-\left\langle Y_{k}, \nabla_{X_{j}}\left(g_{t} Y_{t}\right)\right\rangle\right) \\
& =\sum_{t}\left\langle\nabla_{X_{j}} Y_{k}, g_{t} Y_{t}\right\rangle=\left\langle\nabla_{X_{j}} Y_{k}, \sum_{t} g_{t} Y_{t}\right\rangle .
\end{aligned}
$$

Repetindo a conta anterior para $\left\langle\nabla_{Y_{k}} X_{j}, Z\right\rangle$ obtemos:

$$
\left\langle\nabla_{Y_{k}} X_{j}, Z\right\rangle=\left\langle\nabla_{Y_{k}} X_{j}, \sum_{l} f_{l} X_{l}\right\rangle
$$

Por outro lado, como a conexão é simétrica, temos:

$$
\nabla_{X_{j}} Y_{k}-\nabla_{Y_{k}} X_{j}=\left[X_{j}, Y_{k}\right]=0
$$

daí,

$$
\begin{aligned}
2\left\langle\nabla_{Y_{k}} X_{j}, Z\right\rangle & =\left\langle\nabla_{X_{j}} Y_{k}, Z\right\rangle+\left\langle\nabla_{Y_{k}} X_{j}, Z\right\rangle \\
& =\left\langle\nabla_{X_{j}} Y_{k}, \sum_{t} g_{t} Y_{t}\right\rangle+\left\langle\nabla_{Y_{k}} X_{j}, \sum_{l} f_{l} X_{l}\right\rangle \\
& =\left\langle\nabla_{Y_{k}} X_{j}, \sum_{t} g_{t} Y_{t}\right\rangle+\left\langle\nabla_{Y_{k}} X_{j}, \sum_{l} f_{l} X_{l}\right\rangle \\
& =\left\langle\nabla_{Y_{k}} X_{j}, \sum_{t} g_{t} Y_{t}+\sum_{l} f_{l} X_{l}\right\rangle=\left\langle\nabla_{Y_{k}} X_{j}, Z\right\rangle
\end{aligned}
$$

e, portanto, $\nabla_{X_{j}} Y_{k}=\nabla_{Y_{k}} X_{j}=0$

Finalmente, se $X^{j}$ e $Y^{j}$ são campos de vetores em $M_{j}$, segue que $\nabla_{X^{j}} Y^{k}=0$ se $j \neq k$. Assim,

$$
\nabla_{X^{1}+X^{2}}\left(Y^{1}+Y^{2}\right)=\nabla_{X^{1}} Y^{1}+\nabla_{X^{2}} Y^{2}=\nabla_{X^{1}}^{1} Y^{1}+\nabla_{X^{2}}^{2} Y^{2}
$$

Definição A.10. Seja $M$ uma variedade Riemanniana e $\nabla$ sua conexão de Levi-Civita. Uma curva suave $\gamma: I \rightarrow M$ é uma geodésica se $\nabla_{\dot{\gamma}(t)} \dot{\gamma}(t)=0$ para todo $t$.

Temos então o seguinte corolário da proposição anterior 
Corolário A.11. Sejam $M_{1}, \ldots, M_{n}$ variedades Riemanianas, e $\gamma_{j}: I \rightarrow M_{j}$ geodésicas. Então, $\gamma: I \rightarrow M_{1} \times \cdots \times M_{n}$ definida por $\gamma(t)=\left(\gamma_{1}(t), \ldots, \gamma_{n}(t)\right)$ é geodesica de $M_{1} \times \cdots \times M_{n}$ com a métrica produto.

Demonstração: De fato, se $\nabla^{j}$ é a conexão de $M_{j}$, então temos $\nabla_{\dot{\gamma}_{j}(t)}^{j} \dot{\gamma}_{j}(t)=0$. Então, basta notar que se $\nabla$ é a conexão de Levi-Civita do produto, temos

$$
\nabla_{\dot{\gamma}(t)} \dot{\gamma}(t)=\nabla_{\left(\dot{\gamma}_{1}(t), \ldots, \dot{\gamma}_{n}(t)\right)}\left(\dot{\gamma}_{1}(t), \ldots, \dot{\gamma}_{n}(t)\right)=\nabla_{\dot{\gamma}_{1}(t)}^{1} \dot{\gamma}_{1}(t)+\cdots+\nabla_{\dot{\gamma}_{n}(t)}^{n} \dot{\gamma}_{n}(t)=0
$$

Observação A.12. Seja $\gamma(t)=(r \cos \theta(t), r \sin \theta(t))$ uma curva sobre a circunferencia de raio $r$ em $\mathbb{R}^{2}$. Então, segue de (A.8) que

$$
\nabla_{\dot{\gamma}} \dot{\gamma}=\nabla_{\dot{\theta} \partial_{\theta}}\left(\dot{\theta} \partial_{\theta}\right)=\dot{\theta} \ddot{\theta} \partial_{\theta}
$$

Daí, $\gamma$ é geodésica se, e somente se, $\dot{\theta}$ é constante, isto é $\gamma$ percorre o círculo com velocidade constante. 


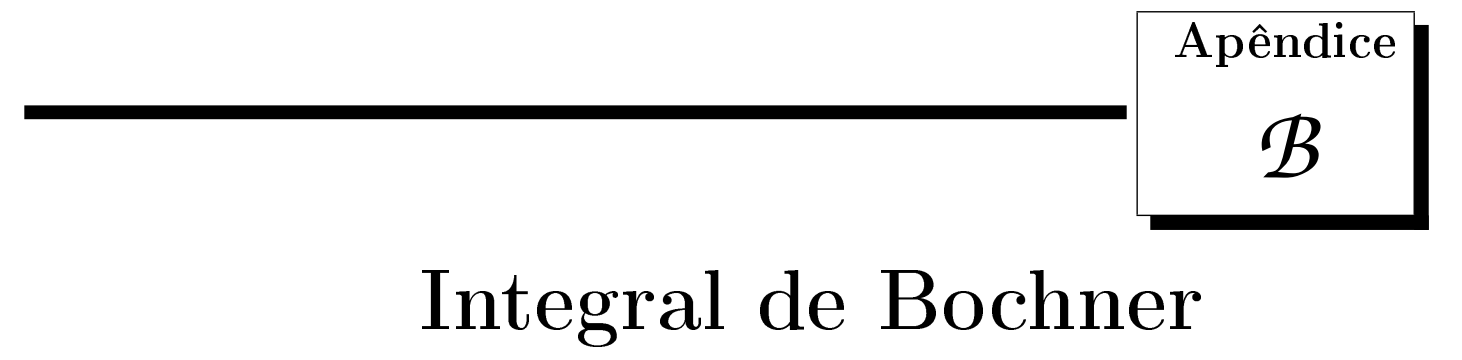

As funções a valores em espaços de Banach desempenham um importante papel dentro da análise matemática e, no desenvolvimento de diversos campos de estudos, tornou-se necessário integrar tais funções; para tal é necessário desenvolver uma teoria de integração apropriada. Escolhemos, pois, a abordagem de S. Bochner por se tratar, em certo sentido, de uma generalização da teoria de integração de Lebesgue.

Assim como no contexto da integral de Lebesgue, precisamos considerar inicialmente um espaço de medida $(\Omega, \mathfrak{U}, \mu)$, onde $\Omega$ é um espaço de Hausdorff, localmente compacto e segundo enumerável, equipado com uma sigma álgebra de borel $\mathfrak{U}$ e uma medida de borel regular $\mu$. Vamos ainda considerar que o nosso espaço seja $\sigma$-finito, isto é, ele pode ser considerado como a união de conjuntos de medida finita. Em particular, vamos considerar $\Omega \subset \mathbb{R}^{n}$ um conjunto de Borel equipado com a $\sigma$-álgebra de Borel usual e a medida de Lebesgue. Seja $X$ um espaço de Banach sobre $\mathbb{C}$ e $X^{*}$ seu dual topológico. Seja $M \subset X$, então a $\sigma$-álgebra de Borel sobre $M$, denotada por $\mathcal{B}(M)$, é a $\sigma$-álgebra gerada pelos conjuntos relativamente abertos de $M$.

Definição B.1. Sejam $A \in \mathfrak{U}$ e $f: A \rightarrow M$ um função. Então $f$ é dita (Borel -) mensurável se $f^{-1}(B) \in \mathcal{B}(A)$ para todo $B \in \mathcal{B}(X)$.

Note que esta é a definição de mensurabilidade usual, então, seguem alguns resultados da teoria usual de medida e integração: A soma de funções mensuráveis é mensurável, assim como o produto por escalar e a composição com funções contínuas. Em particular, se $f$ é mensurável, então $\|f\|$ é mensurável. Além disso, o limite pontual de funções mensurável é mensurável, assim como o sup e o inf de uma coleção enumeravel de funções mensurável é uma função mensurável. 
Definição B.2. Uma função $f: \Omega \rightarrow X$ é dita simples, quando sua imagem é finita e $f^{-1}(p)$ é um conjunto de Borel para todo $p \in X \backslash\{0\}$. Denotando $f(\Omega)=\left\{a_{1}, \ldots, a_{k}\right\}$ e escrevendo $E_{j}=f^{-1}\left(a_{j}\right)$, podemos escrever

$$
f(x)=\sum_{j=1}^{k} a_{j} \chi_{E_{j}}(x)
$$

Esta é chamada a representação padrão de $f$. Uma função simples pode ter diversas repesentações, e de modo geral, as funções simples possuem a seguinte forma:

$$
f(x)=\sum_{j=1}^{n} b_{j} \chi_{A_{j}}(x)
$$

onde cada $A_{j}$ é um conjunto de Borel, mas estes conjuntos não são necessariamente disjuntos. Claramente o espaço das funções simples é um espaço vetorial sobre $\mathbb{C}$.

Além disso, é imediato que as funções simples são mensuráveis.

Observação B.3. Note que, se $f$ é uma função simples com representação padrão $f(x)=$ $\sum_{j=1}^{k} a_{j} \chi_{E_{j}}(x)$, então $\|f\|$ é uma função simples e tem representação padrão

$$
\|f(x)\|=\sum_{j=1}^{k}\left\|a_{j}\right\| \chi_{E_{j}}(x)
$$

Definição B.4. Definimos a integral de uma função simples $f(x)=\sum b_{j} \chi_{A_{j}}$ por

$$
\int_{\Omega} f d \mu=\int_{\Omega} f(x) d \mu(x)=\sum_{j} \mu\left(A_{j}\right) b_{j}
$$

Observação B.5. Note que a definição acima independe da representação de $f$, e isto segue das propriedades aditivas da medida $\mu$. A demonstração desta afirmação é a mesma demonstração da proposição análoga para a integral de Lebesgue.

Note ainda que a integral é um operador linear no espaço das funções simples, isto é, se $f, g: \Omega \rightarrow X$ são funções simples e $\alpha, \beta \in \mathbb{C}$ então

$$
\int_{\Omega} \alpha f(x)+\beta g(x) d \mu(x)=\alpha \int_{\Omega} f(x) d \mu(x)+\beta \int_{\Omega} g(x) d \mu(x)
$$

Novamente, a demonstração deste fato é identica à demonstração para o caso da 
integral de Lebesgue.

Lema B.6. Se $f: \Omega \rightarrow X$ é uma função simples então

$$
\left\|\int_{\Omega} f(x) d \mu(x)\right\| \leq \int_{\Omega}\|f(x)\| d \mu(x)
$$

Demonstração: Suponha que $f$ tenha representação padrão $f(x)=\sum_{j} a_{j} \chi_{E_{j}}$. Então, $\|f\|=\sum_{j}\left\|a_{j}\right\| \chi_{E_{j}}$ e, portanto,

$$
\left\|\int_{\Omega} f(x) d \mu(x)\right\|=\left\|\sum_{j} a_{j} \mu\left(E_{j}\right)\right\| \leq \sum_{j}\left\|a_{j}\right\| \mu\left(E_{j}\right)=\int_{\Omega}\|f(x)\| d \mu(x) .
$$

Como no caso da integral de Lebesgue, queremos estender a noção de integrabilidade a uma classe maior de funções, e faremos isso tomando o limite de funções simples. Ocorre porém, que no caso da integral de Bochner, é necessária uma condição de separabilidade.

Lema B.7. Seja $f: \Omega \rightarrow X$ uma função, então são equivalentes

1. Existe uma sequência $\left(f_{k}\right)_{k \in \mathbb{Z}_{+}}$de funções simples $f_{k}: \Omega \rightarrow X$ tal que $f_{k}(x) \rightarrow f(x)$ $\mathrm{em} \Omega$.

2. $f$ é mensurável e $f(\Omega) \subset X$ é separavel.

Se uma destas afirmações for verdadeira, então podemos tomar a sequência $f_{k}$ tal que $\left\|f_{k}(x)\right\| \leq 2\|f(x)\|$.

Demonstração: Suponha que (1) seja válido, então $f$ é o limite pontual de funções mensuraveis e, portanto, é mensurável. Além disso, escrevendo cada $f_{k}$ em sua representação padrão

$$
f_{k}=\sum_{n=1}^{N_{k}} a_{k, n} \chi_{E_{k, n}}(x)
$$

temos que o espaço gerado pelas combinações lineares racionais dos valores $\left\{a_{k, n} ; n=1, \ldots, N_{k}\right\}$ é denso na imagem de $f$ e, portanto, $f(\Omega)$ é separavel.

Reciprocamente, seja $\left\{y_{1}, y_{2}, \ldots\right\}$ um subconjunto denso de $f(\Omega)$. Ampliando este conjunto por no máximo um número enumeravel de pontos, podemos supor que $y_{j} \neq 0$ 
para todo $j \in \mathbb{Z}_{+}$. Defina

$$
\tilde{A}_{k}^{N}=\left\{x \in \Omega ;\|f(x)\| \geq \frac{1}{N} \text { e }\left\|f(x)-y_{k}\right\|<\frac{1}{N}\right\}
$$

Como $f$ é mensurável, temos que $\{x ;\|f(x)\| \geq \varepsilon>0\}$ é um conjunto de Borel e, analogamente, $\{x ;\|f(x)-y\|<\varepsilon\}$ também o é, de modo que $\tilde{A}_{k}^{N}$ é a interseção de conjuntos de Borel, portanto, também é um conjunto de Borel. Definindo ainda

$$
A_{1}^{N}=\tilde{A}_{1}^{N} \quad \text { e } \quad A_{k}^{N}=\tilde{A}_{k}^{N} \backslash \bigcup_{j=1}^{k-1} A_{j}^{N}
$$

obtemos uma sequencia de conjuntos de Borel disjuntos tais que

$$
\bigcup_{k \in \mathbb{Z}_{+}} A_{k}^{N}=\bigcup_{k \in \mathbb{Z}_{+}} \tilde{A}_{k}^{N}=\left\{x \in \Omega ;\|f(x)\| \geq \frac{1}{N} \text { e }\left\|f(x)-y_{k}\right\|<\frac{1}{N} \text { para algum } k\right\} ;
$$

da densidade de $\left\{y_{1}, \ldots, y_{k}, \ldots\right\}$ segue que

$$
\bigcup_{k \in \mathbb{Z}_{+}} A_{k}^{N}=\left\{x \in \Omega ;\|f(x)\| \geq \frac{1}{N}\right\}
$$

Defina, então, as seguintes funções

$$
F_{N}(x)=\left\{\begin{array}{ll}
y_{k_{N, x}} & \text { se } x \in \bigcup_{j, M=1}^{N} A_{j}^{M} \\
0 & \text { caso contrário }
\end{array},\right.
$$

onde $k_{N, x}$ é determinado da seguinte maneira:

Seja $M_{n}(x)$ o maior inteiro em $\{1, \ldots, N\}$ tal que $x \in \bigcup_{j=1}^{N} A_{j}^{M_{N}(x)}$. Utilizando o fato de que os conjuntos $\left\{A_{j}^{M} ; j=1,2, \ldots\right\}$ são disjuntos para cada $M$ fixado, defina $k_{N, x}$ como o único inteiro em $\{1, \ldots, N\}$, tal que $x \in A_{k_{N, x}}^{M_{N}(x)}$.

Note que, para cada $N \in \mathbb{Z}_{+}, F_{M}$ tem imagem finita e, além disso, por construção, a imagem inversa de cada um destes pontos é um conjunto de Borel; logo, cada $F_{N}$ é uma função simples. Mais ainda, para $x \in A_{k_{N, x}}^{M_{N}(x)}$ temos

$$
\left\|f_{N}(x)\right\|=\left\|y_{k_{N, x}}\right\| \leq\left\|y_{k_{N, x}}-f(x)\right\|+\|f(x)\| \leq \frac{1}{M_{N}(x)}+\|f(x)\| \leq 2\|f(x)\| .
$$


Finalmente vamos mostrar que $F_{N}$ converge à $f$ pontualmente. Para isso, seja $x \in \Omega$ e suponha que $f(x) \neq 0$. Seja $\varepsilon>0$ e escolha $N_{0} \in \mathbb{Z}_{+}$tal que $\min \{\|f(x)\|, \varepsilon\}>\frac{1}{N_{0}}$. Segue de (B.0.1) que

$$
x \in \bigcup_{k \in \mathbb{Z}_{+}} A_{k}^{N_{0}}
$$

mas como os conjuntos $A_{k}^{N_{0}}$ são disjuntos, então existe um único $k_{0}$ tal que $x \in A_{k_{0}}^{N_{0}}$. Seja agora $N \geq \max \left\{N_{0}, k_{0}\right\}$, então,

$$
x \in \bigcup_{k, M=1}^{N} A_{k}^{M},
$$

pois $A_{k_{0}}^{N_{0}}$ aparece nesta união. Além disso, $N_{0} \leq M_{N}(x) \leq N$, pois $M_{N}(x)$ é definido como o maior inteiro em $\{1, \ldots, N\}$ tal que $x \in \cup_{k=1}^{N} A_{k}^{M_{N}(x)}$ e $N_{0}$ satisfaz esta propriedade. Por outro lado, como $x \in A_{k_{N, x}}^{M_{N}(x)}$ temos

$$
\left\|f(x)-F_{N}(x)\right\|=\left\|f(x)-y_{k_{N, x}}\right\| \leq \frac{1}{M_{N}(x)} \leq \frac{1}{N_{0}} \leq \varepsilon
$$

No caso em que $f(x)=0$ temos que $x$ não pertence a nenhum $\operatorname{dos} A_{k}^{N}$ de modo que $F_{N}(x)=0$ e, portanto, a convergência pontual é trivial, concluindo a demonstração do lema.

Este lema sugere a seguinte definição.

Definição B.8. Uma função $f: \Omega \rightarrow X$ é dita Bochner Mensurável (ou Mensurável segundo Bochner, ou ainda fortemente mensurável), quando existe uma sequência de funções simples $g_{n}: \Omega \rightarrow X$ tal que

$$
\left\|g_{n}(x)-f(x)\right\| \rightarrow 0 \quad \text { q.t.p em } \Omega
$$

onde q.t.p significa "exceto em um conjunto de medida nula", ou ainda "para quase todo ponto". Em particular, toda função simples é Bochner mensurável.

Observação B.9. Diversas propriedades acerca da mensurabilidade segundo Bochner das funções podem ser obtidas ao copiar as demonstrações equivalentes para a mensurabilidade segundo Lebesgue. Entre elas, destacamos as seguintes: se $f, g: \Omega \rightarrow X$ e $h: \Omega \rightarrow \mathbb{C}$ são Bochner Mensuráveis, então $f+g$ e $h \cdot g$ também o são. Além disso, 
se $k: X \rightarrow Y$ é uma função contínua entre espaços de Banach, então $k \circ f: \Omega \rightarrow Y$ é Bochner Mensurável. Em particular, $\|f\|$ é mensurável.

Lema B.10. Seja $f: \Omega \rightarrow X$ uma função mensurável segundo Bochner, e suponha que existam duas sequências $g_{n}, h_{n}: \Omega \rightarrow X$ de funções simples, tais que $\left\|f-g_{n}\right\|$ e $\left\|f-h_{n}\right\|$ são mensuráveis e

$$
\lim _{n \rightarrow \infty} \int_{\Omega}\left\|f(x)-g_{n}(x)\right\| d \mu(x)=\lim _{m \rightarrow \infty} \int_{\Omega}\left\|f(x)-h_{m}(x)\right\| d \mu(x)=0 .
$$

Então, para cada $E \in \mathfrak{U}$ temos

$$
\lim _{n \rightarrow \infty} \int_{E} g_{n}(x) d \mu(x)=\lim _{m \rightarrow \infty} \int_{E} h_{m}(x) d \mu(x) .
$$

\section{Demonstração:}

Sejam $g_{n}$ e $h_{n}$ as sequências do enunciado e $E \in \mathfrak{U}$, então

$$
\begin{aligned}
\left\|\int_{E} g_{n} d \mu-\int_{E} g_{m} d \mu\right\| & =\left\|\int_{E}\left(g_{n}-g_{m}\right) d \mu\right\| \\
& \leq \int_{E}\left\|g_{n}-g_{m}\right\| d \mu \leq \int_{\Omega}\left\|g_{n}-g_{m}\right\| d \mu \\
& \leq \int_{\Omega}\left\|g_{n}-f\right\| d \mu+\int_{\Omega}\left\|f-g_{m}\right\| d \mu \stackrel{n, m \rightarrow \infty}{\longrightarrow} 0
\end{aligned}
$$

de modo que $\left(\int_{E} g_{n} d \mu\right)_{n \in \mathbb{Z}_{+}}$é uma sequência de Cauchy em $X$ e, portanto, converge. Analogamente, mostramos que $\left(\int_{E} h_{m} d \mu\right)_{m \in \mathbb{Z}_{+}}$é convergente. Finalmente, a desigualdade

$$
\left\|\int_{E} g_{n} d \mu-\int_{E} h_{n} d \mu\right\| \leq \int_{\Omega}\left\|g_{n}-f\right\| d \mu+\int_{\Omega}\left\|f-h_{n}\right\|
$$

implica que

$$
\lim _{n \rightarrow \infty} \int_{E} g_{n}(x) d \mu(x)=\lim _{m \rightarrow \infty} \int_{E} h_{m}(x) d \mu(x)
$$

Definição B.11. Dizemos que uma função $f: \Omega \rightarrow X$ é Bochner integrável se existem 
funções simples $g_{n}$ tais que $g_{n}(x) \rightarrow f(x)$ q.t.p em $\Omega$ e

$$
\lim _{n \rightarrow \infty} \int_{\Omega}\left\|g_{n}(x)-f(x)\right\| d \mu(x)=0
$$

Quando $f$ for Bochner integravel, definimos sua integral por

$$
\int_{\Omega} f d \mu=\lim _{n \rightarrow \infty} \int_{\Omega} g_{n}(x) d \mu(x)
$$

Observação B.12. É imediato do lema anterior que a integral de Bochner de uma função Bochner integraável está bem definida. Além disso, podemos novamente copiar a demonstração no caso da integral de lebesgue para demonstrar que o espaço das funções Bochner integráveis é um espaço vetorial e a integral de Bochner age como um operador linear neste espaço.

Lema B.13. Uma função $f: \Omega \rightarrow X$ é Bochner integrável se, e somente se, $\|f\|: \Omega \rightarrow \mathbb{R}$ for mensurável e Lebesgue integravel.

Demonstração: Se $f$ é Bochner integrável, então existem funções simples $g_{n}$ tais que $g_{n}(x) \rightarrow f(x)$ q.t.p em $\Omega \mathrm{e}$

$$
\lim _{n \rightarrow \infty} \int_{\Omega}\left\|g_{n}(x)-f(x)\right\| d \mu(x)=0
$$

Como $f$ é mensurável (pois é o limite pontual de funções mensuráveis), segue que $\|f\|$ também é mensurável. Além disso,

$$
\int_{\Omega}\|f\| d \mu \leq \int_{\Omega}\left\|f-g_{n}\right\| d \mu+\int_{\Omega}\left\|g_{n}\right\| d \mu<\infty
$$

logo $\|f\|$ é integrável.

Reciprocamente, suponha que $f$ é mensurável e satisfaz $\int_{\Omega}\|f\| d \mu<\infty$. Segue do Lema B.7 que existe uma sequência de funções simples $f_{k}$ que converge pontualmente para $f$ e satisfaz $\left\|f_{k}(x)\right\| \leq 2\|f(x)\|$. Então, a sequência $g_{k}(x)=\left\|f_{k}(x)-f(x)\right\|$ é uma sequência de funções mensuráveis que converge pontualmente para zero e satisfaz $\left\|g_{k}(x)\right\| \leq 3\|f(x)\|$. Do teorema da convergência dominada de Lebesgue temos

$$
\lim _{k \rightarrow \infty} \int_{\Omega} g_{k}(x) d \mu(x)=0
$$


Finalmente, observe que as funções Bochner integráveis satisfazem

$$
\left\|\int_{\Omega} f(x) d \mu(x)\right\| \leq \int_{\Omega}\|f(x)\| d \mu(x) .
$$

De fato, suponha que $f$ seja Bochner integrável. Então, existe uma sequencia de funções simples $g_{n}$ que aproxima $f$. Logo,

$$
\begin{aligned}
\left\|\int_{\Omega} f(x) d \mu(x)\right\| & =\left\|\lim _{n \rightarrow \infty} \int_{\Omega} g_{n}(x) d \mu(x)\right\|=\lim _{n \rightarrow \infty}\left\|\int_{\Omega} g_{n}(x) d \mu(x)\right\| \\
& \leq \lim _{n \rightarrow \infty} \int_{\Omega}\left\|g_{n}(x)\right\| d \mu(x)=\int_{\Omega}\|f(x)\| d \mu(x) .
\end{aligned}
$$

Lema B.14. Seja $(E,\|\cdot\|)$ um espaço de Banach e $F: \mathbb{R}^{n} \rightarrow E$ função contínua com suporte compacto. Então,

$$
\left\|\int F(y) d y\right\| \leq \int\|F(y)\| d y .
$$

Demonstração: Basta observar que se $F$ é contínua e tem suporte compacto, então $\|F\|$ é mensurável e integrável. Portanto, $F$ é bochner integrável e segue o resultado. 


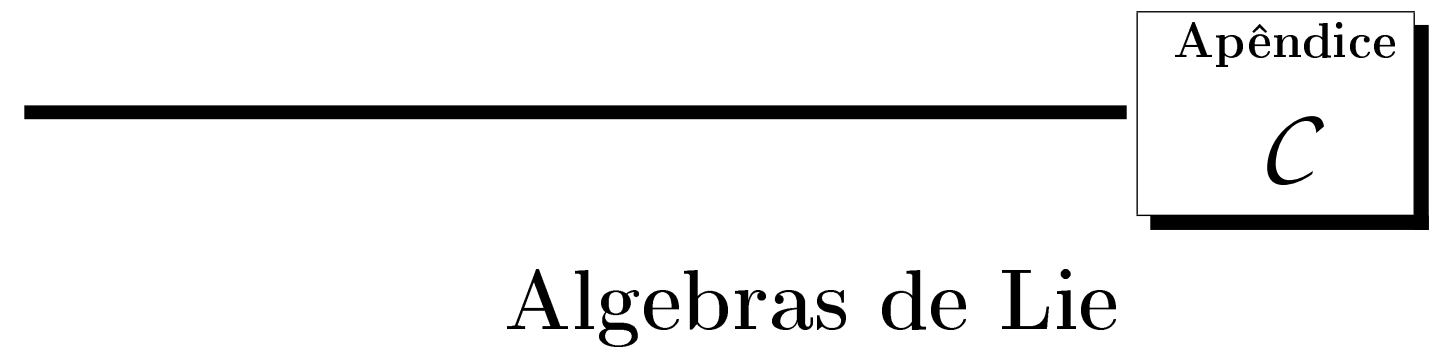

Queremos demonstrar neste apendice o Teorema 6.6. Para isso faremos uso de certos resultados da teoria de representação de álgebras de lie. No que se segue, faremos uma breve introdução aos conceitos básicos da teoria, a fim de enunciar os resultados necessários, a saber, o Teorema de Weyl, e a classificação das representações irredutiveis de $\mathfrak{s l}_{2}(\mathbb{C})$.

\section{C.1 Noções gerais}

Definição C.1. Uma algebra de Lie, é um espaço vetorial $\mathbb{L}$, munido de uma operação bilinear $[\cdot ; \cdot]: \mathbb{L} \times \mathbb{L} \rightarrow \mathbb{L}$, chamada de Colchete de Lie,que satisfaz as seguintes propriedades:

- $[x ; x]=0$ para todo $x \in \mathbb{L}$

- $[x,[y, z]]+[z,[x, y]]+[y,[z, x]]=0$

A segunda propriedade do Colchete de Lie é chamada Identidade de Jacobi.

Observação C.2. A primeira propriedade do Colchete de Lie e a bilinearidade implicam $[x, y]+[y, x]=0$ para quaisquer $x, y \in \mathbb{L}$.

Definição C.3. Um homomorfismo de Algebras de Lie é uma aplicação $\phi: \mathbb{L} \rightarrow \mathbb{L}^{\prime}$ entre algebras de Lie, que preservam o colchete, isto é:

$$
\phi\left([x, y]_{\mathbb{L}}\right)=[\phi(x), \phi(y)]_{\mathbb{L}^{\prime}}
$$


Um isomorfismo de Algebras de Lie, é um homomorfismo de algebras de lie bijetor.

Os mais importantes exemplos de Algebras de Lie são subespaços vetoriais de $M_{n}(\mathbb{F})$ (sendo $\mathbb{F}$ um corpo qualquer) com a operação bilinear dada pelo comutador $[A, B]=A B-B A$.

Vejamos algumas Álgebras de Lie de interesse. Seja $V$ um espaço vetorial sobre $\mathbb{F}$ com dimensão finita.

- $\mathfrak{g l}(V)$ : É a álgebra formada pelos endomorfismos de um espaço vetorial $V$. O colchete é dado por $[T, S]=T \circ S-S \circ T$. Note que $\mathfrak{g l}(V)$ também faz sentido quando $V$ tem dimensão infinta. Quando $V$ tem dimensão finita, $\mathfrak{g l}(V)$ pode ser canônicamente identificado com $M_{n}(\mathbb{F})$, sendo $\operatorname{dim} V=n$.

- $\mathfrak{s l}(V)$ : subalgebra de $\mathfrak{g l}(V)$ formada pelos endomorfismo de $V$ com traço zero.

Sejam $S, M$ subconjuntos de $\mathbb{L}$, iremos denotar $[S, M]=\{[s, m] ; s \in S ; m \in M\}$

Definição C.4. Um ideal $\mathbb{I}$ em uma Álgebra de Lie $\mathbb{L}$ é uma subalgebra que satisfaz $[\mathbb{I} ; \mathbb{L}] \subseteq \mathbb{I}$

Os mais importantes exemplos de ideais de uma álgebra $\mathbb{L}$ são:

- $Z(\mathbb{L})=\{x \in \mathbb{L} ;[x, \mathbb{L}]=0\}$, chamado o Centro de $\mathbb{L}$

- $[\mathbb{L}, \mathbb{L}]$, chamado a álgebra derivada de $\mathbb{L}$.

- Seja $\phi: \mathbb{L} \rightarrow \mathbb{L}^{\prime}$ um homomorfismo de Álgebras de Lie, então $\operatorname{Ker} \phi$ é um ideal de $\mathbb{L}$

Definição C.5. Dizemos que $\mathbb{L}$ é uma Álgebra de Lie simples se $\mathbb{L}$ não possui ideais não triviais.

No caso particular em que $V$ é um espaço vetorial sobre $\mathbb{C}$ de dimensão 2. Vamos denotar $\mathfrak{s l}(V)$ por $\mathfrak{s l}_{2}(\mathbb{C})$.

Lema C.6. $\mathfrak{s l}_{2}(\mathbb{C})$ é uma Álgebra de Lie simples

Demonstração: Note primeiramente que

$$
x=\left(\begin{array}{ll}
0 & 1 \\
0 & 0
\end{array}\right) ; y=\left(\begin{array}{cc}
0 & 0 \\
1 & 0
\end{array}\right) ; h=\left(\begin{array}{cc}
1 & 0 \\
0 & -1
\end{array}\right)
$$


formam uma base de $\mathfrak{s l}_{2}(\mathbb{C})$ e satisfazem as seguintes relações de comutatividade:

$$
[x, y]=h \quad ; \quad[h, x]=2 x ;[h, y]=-2 y
$$

Seja agora $\mathbb{I}$ um ideal não nulo de $\mathfrak{s l}_{2}(\mathbb{C})$. Note que $h \in \mathbb{I}$ implica que $[h, x]=2 x \in \mathbb{I}$ e $[y, h]=2 y \in \mathbb{I}$ e, portanto, $\mathbb{I}=\mathfrak{s l}_{2}(\mathbb{C})$. Vamos então mostrar que se $\mathbb{I}$ é ideal não nulo então $h \in \mathbb{I}$. Seja $A x+B y+C h$ um elemento não nulo qualquer de $\mathbb{I}$ e suponha que $A \mathrm{e}$ $B$ não sejam ambos nulos. Então:

$$
\begin{aligned}
& \mathbb{I} \ni[[A x+B y+C h, h], y]=[2 A x-2 B y, y]=2 A h \\
& \mathbb{I} \ni[[A x+B y+C h, h], x]=[2 A x-2 B y, x]=2 B h
\end{aligned}
$$

Logo $h \in \mathbb{I}$.

Definição C.7. Uma representação de uma Álgebra de Lie $\mathbb{L}$ é um homomorphismo de Álgebras de Lie $\varphi: \mathbb{L} \rightarrow \mathfrak{g l}(V)$ para algum espaço vetorial $V$. Iremos focar no caso em que $V$ tem dimensão finita.

Sejam $\mathbb{L}$ uma Álgebra de Lie, $V$ um espaço vetorial (sobre o mesmo corpo que $\mathbb{L}$ ) e $\psi: \mathbb{L} \times V \rightarrow V$ uma aplicação bilinear que satisfaça a seguinte propriedade:

$$
\psi([x, y], v)=\psi(x, \psi(y, v))-\psi(y, \psi(x, v))
$$

Então $V$ é dito um $\mathbb{L}$-módulo. Em geral, iremos denotar a aplicação bilinear $\psi$ por um ponto: $\psi(x, v)=x \cdot v$. Assim, a condição acima é reescrita por:

$$
[x, y] \cdot v=x \cdot(y \cdot v)-y \cdot(x \cdot v)
$$

Definição C.8. Uma aplicação $\Psi: V \rightarrow W$ é um homomorfismo de $\mathbb{L}$-módulos, se é uma aplicação linear que satisfaz $\Psi(x \cdot v)=x \cdot \Psi(v)$. Um isomorfismo de $\mathbb{L}$-módulos é um homomorfismo bijetor.

Um $\mathbb{L}$-módulo $V$ é dito irredutive, se ele não possui submódulo não trivial. Ele é dito completamente redutivel se pode ser escrito como a soma direta de submódulos irredutiveis. 
Note que podemos associar a cada $\mathbb{L}$-módulo $V$ uma representação de $\mathbb{L}$ definida por:

$$
\begin{aligned}
\varphi: \mathbb{L} & \rightarrow \mathfrak{g l}(V) \\
x & \mapsto \varphi(x)(v)=x \cdot v
\end{aligned}
$$

Reciprocamente, a cada representação $\varphi: \mathbb{L} \rightarrow \mathfrak{g l}(V)$ podemos associar um $\mathbb{L}$-módulo $V$, cuja operação bilinear é definida por $x \cdot v=\varphi(x)(v)$. Com isso, podemos falar de representações de $\mathbb{L}$ irredutiveis e completamente redutíveis.

Seja $\mathbb{L}$ uma álgebra de Lie, e defina a seguinte sequência de ideáis:

$$
\mathbb{L}^{0}=\mathbb{L} \quad ; \quad \mathbb{L}^{(i+1)}=\left[\mathbb{L}^{(i)} ; \mathbb{L}^{(i)}\right]
$$

Note que $\mathbb{L}^{(j+1)} \subset \mathbb{L}^{(j)}$. Diremos então que uma Álgebra de Lie $\mathbb{L}$ é solúvel quando $\mathbb{L}^{(j)}=0$ para algum $j$. $\mathbb{L}$ é dita semisimples se não possui ideal não trivial solúvel. Em particular, uma álgebra simples é semisimples.

Teorema C.9. Se $\sigma: \mathbb{L} \rightarrow \mathfrak{g l}(V)$ é uma representação finita de uma Álgebra de Lie semisimples $\mathbb{L}$, então $\sigma$ é completamente redutivel.

Em particular, toda representação finita de $\mathfrak{s l}_{2}(\mathbb{C})$ é completamente redutivel.

Consideremos agora um $\mathfrak{s l}_{2}(\mathbb{C})$-módulo $V(\operatorname{dim}(V)<\infty)$ qualquer e $\{x, y, h\}$ a base standard de $\mathfrak{s l}_{2}(\mathbb{C})$. É possivel mostrar que $h$ age diagonalmente sobre $V$. Podemos então considerar $V$ como a soma dos autoespaços de $h$ :

$$
V=\bigoplus V_{\lambda} \quad ; \quad V_{\lambda}=\{v \in V ; h \cdot v=\lambda v\}
$$

Teorema C.10. Considere $\mathbb{L}=\mathfrak{s l}_{2}(\mathbb{C})$ e $V$ um $\mathbb{L}$-módulo irredutivel. Seja ainda $V_{\lambda}$ definido acima. As seguintes afirmações são verdadeiras:

(a) $V$ se escreve como a soma direta dos autoespaços $V_{\mu}, \mu=m, m-2, \ldots, 2-m,-m$ $e \operatorname{dim}\left(V_{\mu}\right)=1, \operatorname{com} \operatorname{dim}(V)=m+1$.

(b) Seja $v_{0}$ autovetor associado a um autovalor maximal $m$ (maior parte real). Então $x \cdot v_{0}=0$ e $\left\{v_{0}, \ldots, v_{m}\right\}$ definidos por

$$
v_{j}=\frac{1}{j !} y^{j} \cdot v_{0} \forall j>0
$$


formam uma base de autovetoresde $V$ associada $a h$.

(c) A atuação de $\mathbb{L}$ em $V$ com relação à esta base de autovetores é dada explicitamente por

$$
\begin{aligned}
& x \cdot v_{j}=(m-j+1) v_{j-1} ; \quad\left(v_{-1}=0\right) \\
& h \cdot v_{j}=(m-2 j) v_{j} \\
& y \cdot v_{j}=(j+1) v_{j+1} ; \quad\left(v_{m+1}=0\right)
\end{aligned}
$$

Em particular, existe no máximo um (a menos de isomorfismo) $\mathbb{L}$-módulo irredutivel de dimensão $m+1$ para cada $m \geq 0$.

\section{C.2 Demonstração do Teorema 6.6}

Seja então $\rho: \mathfrak{s l}_{2}(\mathbb{C}) \rightarrow \mathfrak{g l}(V)$ a única representação irredutivel de dimensão $m+1 \mathrm{e}$ defina $\sigma: \mathfrak{s l}_{2}(\mathbb{C}) \rightarrow \mathfrak{g l}(V)$ por:

$$
\begin{aligned}
\sigma(x) & =\frac{1}{2}(\rho(h)-\rho(x)+\rho(y)) \\
\sigma(h) & =\rho(x)+\rho(y) \\
\sigma(y) & =\frac{1}{2}(\rho(h)+\rho(x)-\rho(y))
\end{aligned}
$$

Vejamos que $\sigma$ é uma representação. Basta mostrar que, para $a, b$ elementos da base de $\mathfrak{s l}_{2}(\mathbb{C})$, vale $\sigma([a, b])=[\sigma(a), \sigma(b)]$.

- $a=b=x$ (os caso $a=b=y$ e $a=b=h$ são análogos)

$$
\begin{aligned}
{[\sigma(x), \sigma(x)] } & =\frac{1}{4}[\rho(h-x+y), \rho(h-x+y)]=\frac{1}{4} \rho([h-x+y, h-x+y]) \\
& =\frac{1}{4} \rho(0)=0=\sigma(0)=\sigma([x, x])
\end{aligned}
$$

- $a=x, b=y(\mathrm{O}$ caso $a=y$ e $b=x$ é análogo $)$

$$
\begin{aligned}
{[\sigma(x), \sigma(y)] } & =\frac{1}{4}[\rho(h-x+y), \rho(h+x-y)]=\frac{1}{4} \rho([h-x+y, h+x-y]) \\
& =\frac{1}{4} \rho(2[h, x-y])=\frac{1}{4} \rho(2[h, x]+2[y, h])=\rho(x+y)=\sigma(h)=\sigma[x, y]
\end{aligned}
$$


- $a=x, b=h$ (os demais casos são análogos)

$$
\begin{aligned}
{[\sigma(x), \sigma(h)] } & =\frac{1}{2}[\rho(h-x+y), \rho(x+y)]=\frac{1}{2} \rho([h-x+y, x+y]) \\
& =\frac{1}{2} \rho([h, x+y]-2[x, y])=\frac{1}{2} \rho(2 x-2 y-2 h)=-\rho(h-x+y) \\
& =\sigma(-2 x)=\sigma([x, h])
\end{aligned}
$$

Seja agora $w_{0}=v_{0}+v_{1}+\cdots+v_{m}$, então:

$$
\begin{aligned}
& \rho(x) w_{0}=\sum_{j=0}^{m}(m-j+1) v_{j-1}=\sum_{k=0}^{m-1}(m-k) v_{k} \\
& \rho(y) w_{0}=\sum_{j=0}^{m}(j+1) v_{j+1}=\sum_{k=1}^{m} k v_{k}
\end{aligned}
$$

assim,

$$
\sigma(h) w_{0}=\rho(x) w_{0}+\rho(y) w_{0}=\left(\sum_{j=1}^{m-1}((m-j)+j) v_{j}+m v_{0}+m v_{m}\right)=m w_{0}
$$

Do teorema de Weyl (Teorema C.9) segue que $\sigma$ é completamente redutivel, logo existe um submódulo $W$ irredutivel que contem $w_{0}$. Do Teorema C.10 segue que, se $k$ é autovalor $h$ em um submódulo irredutivel, então $k, k-2, \ldots, 2-k,-k$ são autovalores de $h$, logo (C.2.1) implica que $\operatorname{dim}(W) \geq m+1$. Assim $W=V$ e, portanto, $\sigma$ é irredutivel, e $m, m-2, \ldots, 2-m,-m$ são autovalores de $\sigma(h)$. Mas $\sigma(h)$ é dada por:

$$
\sigma(h)=\left(\begin{array}{ccccccc}
0 & m & 0 & \ldots & 0 & 0 & 0 \\
1 & 0 & m-1 & \ldots & 0 & 0 & 0 \\
0 & 2 & 0 & \ddots & & & \\
\vdots & \vdots & \ddots & \ddots & \ddots & \vdots & \vdots \\
& & & \ddots & 0 & 2 & 0 \\
0 & 0 & 0 & \ldots & m-1 & 0 & 1 \\
0 & 0 & 0 & \ldots & 0 & m & 0
\end{array}\right)
$$

Segue então do Lema 6.7 que os autovalores de $T_{0}(m, 1)$ são $i m, i(m-2), \ldots, i(2-$ $m)$, -im. Como $T_{0}(m, b)=b T_{0}(m, 1)$, segue a demonstração do Teorema 6.6. 


\section{C.3 Álgebra de Heisenberg e Grupo de Heisenberg}

Definição C.11. Defina em $\mathbb{R}^{2 n+1}$ uma forma bilinear $[\cdot, \cdot]: \mathbb{R}^{2 n+1} \times \mathbb{R}^{2 n+1} \rightarrow \mathbb{R}^{2 n+1}$ definida por

$$
[(p, q, s),(x, y, t)]=(0,0, p \cdot y-q \cdot x) \quad ; p, q, x, y \in \mathbb{R}^{n}
$$

onde $\cdot$ denota o produto interno usual em $\mathbb{R}^{n}$. Para facilitar a notação, iremos suprimir o ponto e denotamos $a \cdot b=a b$.

Não é dificil verificar que $\left(\mathbb{R}^{2 n+1},[\cdot, \cdot]\right)$ é uma álgebra de Lie. Chamamos esta álgebra de Álgebra de Heisenberg e a denotamos por $\mathfrak{h}_{\mathfrak{n}}$.

Uma maneira mais natural de construir esta álgebra é da seguinte forma: considere os operadores $Q_{j}$ e $P_{j}$ atuando em $\mathcal{S}\left(\mathbb{R}^{n}\right)$

$$
Q_{j} f(x)=x_{j} f(x) \quad P_{j} f(x)=\frac{1}{2 \pi i} \frac{\partial}{\partial x_{j}} f(x)
$$

É fácil ver que $Q_{j}$ e $P_{k}, j, k=1, \ldots, n$ satisfazem as seguintes relações de comutatividade:

$$
\left[Q_{j}, Q_{k}\right]=\left[P_{j}, P_{k}\right]=0 \quad ; \quad\left[P_{j}, Q_{k}\right]=-i \delta_{j, k} I d
$$

Para $p, q \in \mathbb{R}^{n}$ e $t \in \mathbb{R}$ definimos os operadores

$$
p X=\sum_{j=1}^{n} p_{j} Q_{j} ; q D=\sum_{j=1}^{n} q_{j} P_{j} ; t U=-i t I d
$$

e identifiquemos $(p, q, s) \in \mathbb{R}^{n} \times \mathbb{R}^{n} \times \mathbb{R}$ com o operador $p X+q D+s U$. Então,

$$
\begin{aligned}
{[p X+q D+s U, x X+y D+t U] } & =[p X, y D]+[q D, x X]=\sum_{j, k=1}^{n}\left(p_{j} y_{k}\left[Q_{j}, P_{k}\right]+q_{j} x_{k}\left[P_{j}, Q_{k}\right]\right) \\
& =\sum_{j=1}^{n}\left(p_{j} y_{j}-q_{j} x_{j}\right)\left[Q_{j}, P_{j}\right]=-i(p y-q x) I d=(p y-q x) U
\end{aligned}
$$

Podemos então considerar a álgebra de Heisenberg como a ágebra formada pelos operadores da forma $(p X+q D+t U)$ atuando em $\mathcal{S}$. Na verdade, por dualidade, podemos considerar estes operadores atuando em $L^{2}\left(\mathbb{R}^{n}\right)$. 
Queremos encontrar o grupo associado à esta álgebra, e para isso, é conveniente escrever escrever um elemento $(p, q, s) \in \mathfrak{h}_{\mathfrak{n}}$ como uma matriz $(2 n+1) \times(2 n+1)$ :

$$
M(p, q, s)=I d+m(p, q, s)=I d+\left(\begin{array}{ccc}
0 & p & s \\
0 & 0 & q \\
0 & 0 & 0
\end{array}\right)
$$

Consideramos o comutador usual de matrizes temos que

$$
[m(p, q, s), m(x, y, t)]=m(0,0, p y-q x) \quad \text { e } \quad[M(p, q, s), M(x, y, t)]=M(0,0, p y-q x)
$$

Assim, não é difícil ver que $\mathfrak{h}_{\mathfrak{n}} \ni(p, q, s) \mapsto m(p, q, s)$ e $\mathfrak{h}_{\mathfrak{n}} \ni(p, q, s) \mapsto M(p, q, s)$ são na verdade isomorfismos de álgebras de Lie. Note que $m(p, q, s$,$) é nilpotente, mais$ precisamente $m(p, q, s)^{2}=m(0,0, p q)$ e $m(p, q, s)^{k}=0$ para $k \geq 3$

Vamos agora utilizar o mapa exponencial para identificar o grupo de Lie associado à $\mathfrak{h}_{\mathfrak{n}}$. De modo geral o mapa exponêncial é definido utilizando o fluxo de campos vetoriais invariantes pela esquerda, e mapa exponencial é então um diffeormorfismo entre uma vizinhança da origem na álgebra e uma vizinhança da identidade do grupo de Lie associado. Mas quando o mapa exponencial se refere a matrizes, ele coincide com a exponencial de matrizes usual. Como já vimos, podemos identificar $\mathfrak{h}_{\mathfrak{n}}$ com o espaço das matrizes $\left\{m(p, q, s),(p, q, s) \in \mathbb{R}^{s n+1}\right\}$, além disso, da nilpotência das matrizes neste espaço, concluímos que podemos reduzir a exponencial na seguinte soma finita:

$$
\exp (m(p, q, s))=I d+m(p, q, s)+\frac{1}{2} m(p, q, s)^{2}=M\left(p, q, s+\frac{1}{s} p q\right)
$$

de modo que a exponencial é na verdade um isomorfismo global entre $\mathfrak{h}_{\mathfrak{n}}$ e $\left\{M(p, q, s),(p, q, s) \in \mathbb{R}^{2 n+1}\right\}$, este últimmo com a operação de grupo dada pela multiplicação de matrizes usual:

$$
M(p, q, s) M(x, y, t)=M(p+x, q+y, s+t+p y)
$$

Temos então duas maneiras de definir o grupo de Heisenberg. Uma possivel maneira de definir o grupo de Heisenberg é identificar $\mathbb{R}^{2 n+1} \operatorname{com}\{M(A), A \in P\}$ e estabelecer a seguinte operação de grupo:

$$
(p, q, s)(x, y, t)=(p+x, q+y, s+t+p y)
$$


O grupo de Heisenberg realizado desta forma é chamado Grupo de Heisenberg Polarizado e é denotado por $\mathbb{H}_{n}^{\text {pol }}$. A outra maneira utiliza a operação de grupo induzida pela exponencial ${ }^{1}$

$$
(p, q, s)(x, y, t))=\left(p+x, q+y, s+t+\frac{1}{2}(p y-q x)\right)
$$

Este é chamado o Grupo de Heisenberg e é denotado por $\mathbb{H}_{n}$. Note que neste caso, o mapa exponencial é meramente a identidade, e as operações de grupo e de álgebra se relacionam da seguinte maneira:

$$
X Y=X+Y+[X, Y] \quad ; \quad \forall X, Y \in \mathbb{R}^{2 n+1}
$$

onde o produto é definido utilizando a regra (C.3.2) e o comutador é definido por (C.3.1). As realizações do grupo de Heisenberg se relacionam pelo seguinte isomorfismo

$$
\begin{aligned}
\varphi: \mathbb{H}_{n} & \rightarrow \mathbb{H}_{n}^{p o l} \\
(p, q, s) & \mapsto\left(p, q, s+\frac{1}{2} p, q\right)
\end{aligned}
$$

Consideremos agora, para cada $h \in \mathbb{R} \backslash\{0\}$ a representação $p i_{h}$ de $\mathbb{H}_{n}$ dada por $\pi_{h}(p, q, t)=e^{i(h t I d+p X+q h D)}$. Explicitamente,

$$
\begin{aligned}
& \pi_{h}: \mathbb{H}_{n} \rightarrow \operatorname{Aut}\left(L^{2}\left(\mathbb{R}^{n}\right)\right) \\
&(p, q, t) \mapsto \pi_{h}(p, q, t): L^{2} \rightarrow L^{2} \\
& \quad f \mapsto \pi_{h}(p, q, t) f(x)=e^{i\left(t h+p x+\frac{p q h}{2}\right)} f(x+h q)
\end{aligned}
$$

Esta representação recebe o nome de Representação de Schrodinger. Sua representação derivada ${ }^{2}$ também é denotada por $\pi_{h}$.

É facil observar que a representação de Schrodinger não é injetiva, para corrigir este fato, é usual considerarmos o grupo e Heisenberg reduzido

Definição C.12. O grupo quociente

$$
\mathbb{H}_{n}^{\text {red }}=\mathbb{H}_{n} /\{(0,0, k), k \in 2 \pi \mathbb{Z}\}
$$

e chamado de Grupo de Heisenberg Reduzido

\footnotetext{
${ }^{1} \exp (m(p, q, s)) \exp (m(x, y, t))=\exp \left(m\left(p+x, q+y, s+t+\frac{1}{2}(p y-q x)\right)\right)$

${ }^{2}$ Se $\rho$ é uma representação de um grupo de Lie $G$, sua representação derivada é uma representação da álgebra de Lie $g$ associada a $G$.
} 
Continuamos a denotar os seus elementos por $(p, q, t)$, mas estamos supondo agora que $t \in[0,2 \pi / h)$. Podemos então considerar $\pi_{h}$ como uma representação injetiva de $\mathbb{H}_{n}^{r e d}$. Uma simplificação útil e usual é omitir o termo $e^{i h t I d}$ e escrevemos

$$
\pi_{h}(p, q)=\pi_{h}(p, q, 0)=e^{i(p X+h q D)}
$$

Seja então $F \in L^{1}\left(\mathbb{H}_{n}^{r e d}\right)$, a representação integral de $\pi_{h}$ em $\mathbb{H}_{n}^{\text {red }}$ é definida por:

$$
\begin{aligned}
\pi_{h}(F): L^{2}\left(\mathbb{R}^{n}\right) & \rightarrow L^{2}\left(\mathbb{R}^{n}\right) \\
f & \mapsto \int_{\mathbb{H}_{n}^{r e d}} F(g) \pi(g) f d g=\int_{\mathbb{R}^{2 n} \times \mathbb{T}} F(p, q, t) \pi_{h}(p, q, t) f d p d q d t
\end{aligned}
$$

onde a integral acima é uma integral de Bochner. Note que $\pi_{h}(F)$ define um operador limitado em $L^{2}\left(\mathbb{R}^{n}\right)$ com $\left\|\pi_{h}(F)\right\| \leq\|F\|_{L^{1}\left(\mathbb{H}_{n}^{r e d}\right)}$. Isto segue do fato de que $\pi_{h}$ é unitário.

Expandindo $F$ em sua série de Fourier na variável $t$, temos

$$
F(p, q, t)=\sum_{k=-\infty}^{\infty} \hat{F}_{k}(p, q) e^{i k h t}
$$

Segue então do teorema da convergência dominada que podemos reescrever a representação integral da seguinte forma:

$$
\begin{aligned}
\pi_{h}(F) & =\int_{\mathbb{R}^{n}} \int_{\mathbb{R}^{n}} \int_{0}^{2 \pi / h} \sum_{k=-\infty}^{\infty} \hat{F}_{k}(p, q) e^{i k h t} \pi_{h}(p, q, t) d p d q d t \\
& =\int_{\mathbb{R}^{n}} \int_{\mathbb{R}^{n}} \int_{0}^{2 \pi / h} \sum_{k=-\infty}^{\infty} \hat{F}_{k}(p, q) e^{i k h t} e^{i h t} \pi_{h}(p, q) d p d q d t \\
& =\sum_{k=-\infty}^{\infty} \int_{\mathbb{R}^{n}} \int_{\mathbb{R}^{n}} \hat{F}_{k}(p, q) \pi_{h}(p, q) d p d q \int_{0}^{2 \pi / h} e^{i(k+1) h t} d t
\end{aligned}
$$

note que para $m \neq 0$ temos

$$
\int_{0}^{2 \pi / h} e^{i m h t} d t=0
$$

portanto

$$
\pi_{h}(F)=\frac{2 \pi}{h} \int_{\mathbb{R}^{n}} \int_{\mathbb{R}^{n}} \hat{F}_{-1}(p, q) \pi_{h}(p, q) d p d q
$$


de modo que na verdade, concluímos que na verdade apenas um dos coeficientes de Fourier de $F$ contribui para o valor de $\pi_{h}(F)$. Podemos assim considerar $\pi_{h}$ como uma representação de $L^{1}\left(\mathbb{R}^{2 n}\right)$

Definição C.13. Seja $F \in L^{1}\left(\mathbb{R}^{2 n}\right)$ e seja $\pi_{h}(p, q)=e^{i(p X+q h D)}: L^{2}\left(\mathbb{R}^{n}\right) \rightarrow L^{2}\left(\mathbb{R}^{n}\right)$. Então definimos a representação integral de $F$ como o operador

$$
\begin{aligned}
& \tilde{\pi}_{h}(F): L^{2}\left(\mathbb{R}^{n}\right) \rightarrow L^{2}\left(\mathbb{R}^{n}\right) \\
& f \mapsto \tilde{\pi}_{h}(F) f=\frac{2 \pi}{h} \int_{\mathbb{R}^{2 n}} F(p, q) \pi_{h}(p, q) f d p d q=\frac{2 \pi}{h} \int_{\mathbb{R}^{2 n}} F(p, q) e^{i(p X+q h D)} f d p d q \\
&=\frac{2 \pi}{h} \int_{\mathbb{R}^{2 n}} F(p, q) e^{i(p X+q h D)}\left(\frac{h}{2 \pi} \int_{0}^{2 \pi / h} e^{i h t} e^{-i h t} d t\right) f d p d q \\
&=\int_{\mathbb{R}^{2 n}} \int_{0}^{2 \pi / h} e^{-i h t} F(p, q) e^{i(h t+p X+q h D)} f d p d q d t=\pi_{h}(F \otimes \chi)
\end{aligned}
$$

sendo $\chi(t)=e^{-i h t}$

Claramente, $\pi_{h}(F)$ é um operador limitado e satisfaz $\left\|\pi_{h}(F)\right\| \leq\|F\|_{L^{1}\left(\mathbb{R}^{2 n}\right)}$. Escrevendo

$$
K_{F}(x, y)=\frac{2 \pi}{h} \mathcal{F}_{1}^{-1} F\left(\frac{x+y}{4 \pi}, \frac{y-x}{h}\right)
$$

sendo $\mathcal{F}_{j}$ a transformada parcial de Fourier na $j$-ésima variável. Temos:

$$
\begin{aligned}
\int_{\mathbb{R}^{n}} K_{F}(x, y) f(y) d y & =\int_{\mathbb{R}^{n}}\left(\frac{2 \pi}{h} \int_{\mathbb{R}^{n}} F\left(p, \frac{y-x}{h}\right) e^{2 \pi i p\left(\frac{x+y}{4 \pi}\right)} d p\right) f(y) d y \\
& =\frac{2 \pi}{h} \int_{\mathbb{R}^{2 n}} F\left(p, \frac{y-x}{h}\right) e^{2 \pi i \frac{p(y+x)}{4 \pi}} f(y) d p d y \\
& =\frac{2 \pi}{h} \int_{\mathbb{R}^{2 n}} F\left(p, \frac{y-x}{h}\right) e^{\left.i p x+i \frac{p(y-x)}{2}\right)} f(y) d p d y \\
& =\frac{2 \pi}{h} \int_{\mathbb{R}^{2 n}} F(p, q) e^{i\left(p x+\frac{p q h}{2}\right)} f(x+h q) d p d q=\tilde{\pi}_{h}(F) f(x)
\end{aligned}
$$

Ou seja, podemos entender o $\pi_{h}(F)$ como um operador integral, cujo Kernel é dado por $K_{F}$. Isso nos permite generalizar o mapa $\pi_{h}(F)$ de $L^{2}\left(\mathbb{R}^{n}\right)$ para $\mathcal{S}^{\prime}\left(\mathbb{R}^{n}\right)$ utilizando o teorema do Kernel de Schwartz e o fato de que as operações que definem $K_{F}$ podem ser extendidas à $\mathcal{S}^{\prime}\left(\mathbb{R}^{n}\right)$ por dualidade.

Definição C.14. Se $G$ é um grupo de Lie, então, sabemos sua álgebra de lie $g$ pode ser identificada com os campos de vetores em $G$ invariantes pela esquerda. É natural então, considerar operadores de ordens superiores. O conjunto destes operadores é denotado por 
$\mathfrak{U}(g)$ e recebe o nome de "álgebra envelopante universal". Mais precisamente

$\mathfrak{U}(g)=\{P(\partial)$; operador diferencial invariante pela esquerda atuando em $G\}$

O adjetivo "universal" segue da seguinte observação

Observação C.15. Se $A$ é uma álgebra associativa sobre $\mathbb{C}$ e $f: g \rightarrow A$ é uma aplicação $\mathbb{R}$-linear tal que

$$
f([X, Y])=f(X) f(Y)-f(Y) f(X)
$$

então existe um único homomorfismo $\tilde{f}: \mathfrak{U}(g) \rightarrow A$ de algebras complexas associativas, tal que o diagrama abaixo comuta

$$
\begin{aligned}
& g \hookrightarrow \mathfrak{U}(g) \\
& \mathrm{f} \searrow \downarrow \tilde{f} \\
& \text { A }
\end{aligned}
$$

Esta representação é extendida à "álgebra envelopante universal" denotada por $\mathfrak{U}\left(\mathfrak{h}_{\mathfrak{n}}\right)$. 


\section{Referências Bibliográficas}

ESCHRIG, H. Topology and Geometry for Physics. Berlin: Springer-Verlag, 2011.

FOlland, G. B. Harmonic Analysis in Phase Space. Princeton, Princeton U. Press, 1989.

—. A course in Abstract Harmonic Analysis. Florida: Taylor Francis, 1994.

GUILLEMIN, V.; SCHAEFFER, D. On a certain class of Fuchsian partial differential equations. Duke Math. J. 44, no. 1, 157 - 199, 1977

HÖRMANDER, L. The analysis of linear partial differential operators. Distribution theory and Fourier analysis, second edition. Berlin: Springer, 1990. v. I

—. The analysis of linear partial differential operators. Differential operators with constant coefficients, reprint of the 1983 original. Berlin: Springer-Verlag, 2005. v.II

HOUNIE, J. Teoria Elementar das Distribuições. Rio de Janeiro: IMPA, 1979.

HUMPHREYS, J. E. Introduction to Lie algebras and representation theory. Second printing, revised. New York-Berlin: Springer-Verlag, 1978

KOVACS, I. SILVER, D.S. WILLIAMS S. G. Determinants of Commuting-Block Matrices. The American Mathematical Monthly. 106, No. 10, 950 - 952, 1999.

LOJASIEWICZ, S. Sur le problème de la division.Studia Math. 18, 87-136, 1959.

LEIPNIK, R. B.; PEARCE, C. E. M. The multivariable Faà di Bruno Formula and multivariable Taylor expansions with explicit integral remainder term. ANZIAM Journal. 48, no 3,327 - 341, 2007.

CARMO, M. P. Geometria Riemanniana. Rio de Janeiro: IMPA, 1988. 
MÜLLER, D.; RICCI, F. Analysis of second order differential operators on Heisenberg groups. I. Invent. Math. 101, no. 3, 545 - 582, 1990

Analysis of second order differential operators on Heisenberg groups. II. J. Funct. Anal. 108, no. 2, 296 - 346, 1992.

MÜLLER, D. Local solvability of first order differential operators near a critical point, operators with quadratic symbols and the Heisenberg group. Comm. Partial Differential Equations. 17, no. 1-2, 305 - 337, 1992

NELSON,E. Topics in Dynamics I: Flows. Princeton: Princeton U. Press, 1969.

SILVA, M. F. Resolubilidade Global de Operadores Diferenciais Parciais Reais de Ordem Um. 2006. 70 f. Tese (Doutorado em Matemática) - Departamento de Matemática Universidade Federal de São Carlos, São Carlos, 2006.

SILVA, M. F.; dos SANTOS FILHO, J.R. Global solvability for first order real linear partial differential operators. Journal of Differential Equations, v. 247, p. 2688-2704, 2009 .

STERNBERG, S. The structure of local homeomorphisms. III. Amer. J. Math. 81,578 $604,1959$.

TREVES, F. On the solvability of vector fields with real linear coefficients. Proc. Amer. Math. Soc. 137, no. 12, 4209 - 4218, 2009. 